\title{
Electrospinning as a route to advanced carbon fibre materials for selected low-temperature electrochemical devices: a review
}

Yue Wen ${ }^{1}$, Matt D. R. Kok ${ }^{1}$, Jorge Pavel Victoria Tafoya ${ }^{2}$, Ana B. Jorge Sobrido ${ }^{2}$, Ellsworth Bell $^{3}$, Jeff T. Gostick ${ }^{3}$, Servann Herou ${ }^{4}$, Philipp Schlee ${ }^{4}$, Maria-Magdalena Titirici ${ }^{4}$, Dan J. L. Brett $^{1}$, Paul R. Shearing ${ }^{1}$, Rhodri Jervis ${ }^{1 *}$

${ }^{1}$ The Electrochemical Innovation Lab, Dept. of Chemical Engineering, University College London, Torrington Place, WC1E 7JE, London, UK

${ }^{2}$ Queen Mary University of London, School of Engineering and Materials Science, Mile End Road, E1 4NS, London, UK

${ }^{3}$ Department of Chemical Engineering, University of Waterloo, Waterloo, ON, Canada

${ }^{4}$ Department of Chemical Engineering, Imperial College London, South Kensington Campus, London, SW7 2AZ, UK

* Corresponding author. E-mail address: rhodri.jervis@ucl.ac.uk

\begin{abstract}
Electrospinning has been proven as a highly versatile fabrication method for producing nanostructured fibres with controllable morphology, of both the fibres themselves and the void structure of the mats. Additionally, it is possible to use heteroatom doped polymers or to include catalytic precursors in the electrospinning solution to control the surface properties of the fibres. These factors make it an ideal method for the production of electrodes and flow media for a variety of electrochemical devices, enabling reduction in mass transport and activation overpotentials and therefore increasing efficiency. Moreover, the use of biomass as a polymer source has recently gained attention for the ability to embed sustainable principles in the materials of electrochemical devices, complementing their ability to allow an increase in the use of renewable electricity via their application. In this review, the historical and recent developments of electrospun materials for application in redox flow batteries, fuel cells, metal air batteries and supercapacitors are thoroughly reviewed, including an overview of the electrospinning process and a guide to best practice. Finally, we provide an outlook for the emerging use of this process in the field of electrochemical energy devices with the hope that the combination of tailored microstructure, surface functionality and computer modelling will herald a new era of bespoke functional materials that can significantly improve the performance of the devices in which they are used.
\end{abstract}

Keywords: Electrospinning; Redox flow batteries; Fuel cells; Metal-air batteries; Supercapacitors. 


\section{Highlights}

- A comprehensive review of electrospinning for selected electrochemical devices

- Electrospinning maximises surface area and controls mass transport in redox flow batteries

- Advanced gas diffusion layers can be manufactured for fuel cells and metal air batteries

- Electrospinning can anchor catalysts and have intrinsic activity via heteroatom doping

- High surface area and controlled porosity show promise for supercapacitors

- Use of biomass precursors provides a route for sustainable electrospinning

- This review provides best practice and detailed perspectives for the field

Author biographies

Yue Wen received her BSc degree in Food Science from University College Cork in 2017 and MSc degree in Chemical Engineering from University College London in 2018. She is a Ph.D. candidate at University College London since 2019 under the supervision of Prof. Dan Brett and Dr. Rhodri Jervis. Her research focus is electrospinning of electrode materials and catalysts for redox flow batteries. She holds a scholarship from the Chinese Scholarship Council.

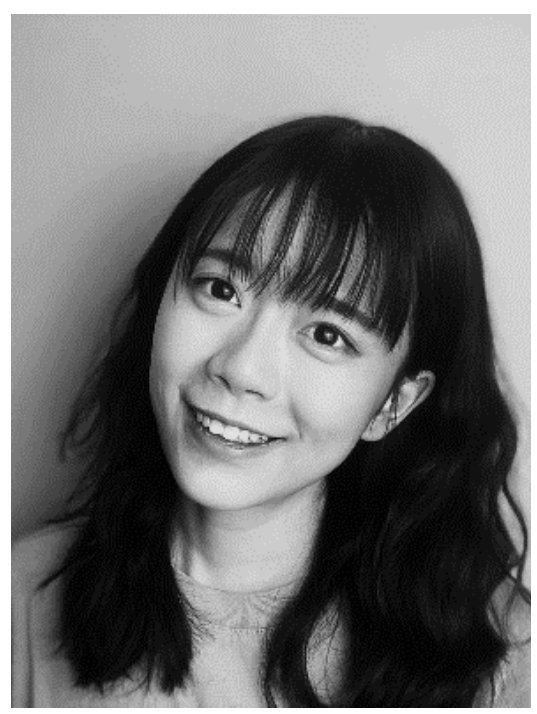

Matt Kok received his Ph.D. Chemical Engineering from McGill University in 2018 with Professor Jeff Gostick where his work focused on electrospinning custom electrodes for flow batteries. He joined the Electrochemical Innovation Lab at University College London shortly after in 2018 where his work has focused on X-ray CT and image analysis for lithium-ion cells. 


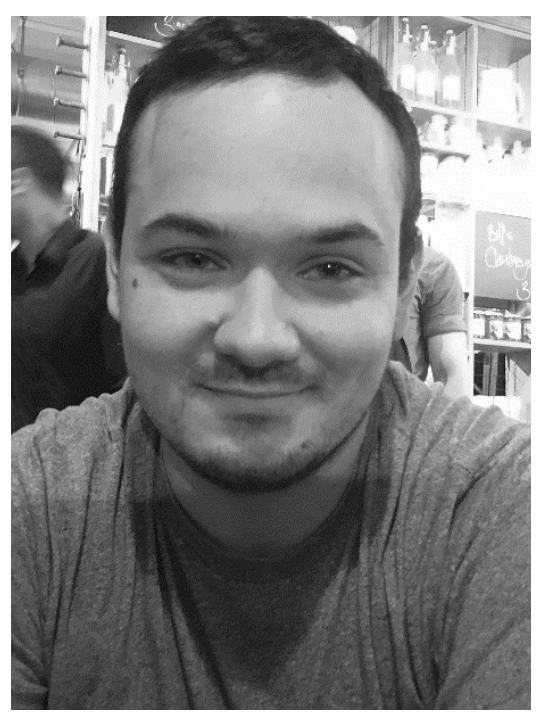

J. Pavel Victoria Tafoya graduated from his bachelor's in chemical engineering during summer of 2007. From 2008 until 2018 he worked as an academic in the University of Michoacan (UMSNH) lecturing undergraduate students and collaborating with researchers characterising optical properties of materials. Between 2012 and 2015 he studied his MSc in physical engineering specialising in electrochemical processes for ores lixiviation. From 2018 is pursuing a $\mathrm{PhD}$ on material science specialising on carbon-based materials for oxygen electrocatalysis under the supervision of Dr. Ana Belén Jorge in Queen Mary University of London.

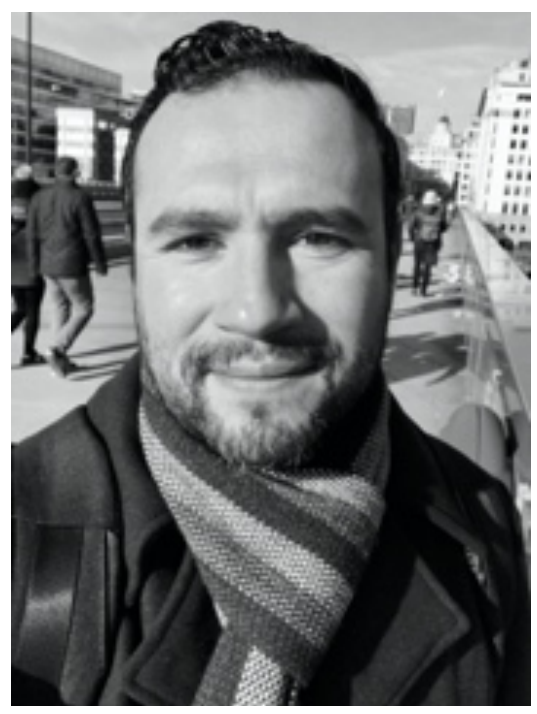

Ana Belén Jorge graduated in Chemistry in the Canary Islands in 2004. She obtained her Ph.D. in Materials Science from the Instituto de Ciencia de Materiales-Universidad Autonoma de Barcelona in 2009. After some time in industry, Ana took a postdoctoral position at University College London investigating new carbon nitrides for energy applications. Since 2016, she is an academic at Queen Mary University of London, first as Lecturer and since 2019, as Senior Lecturer. Her research focuses on creating new sustainable materials for energy applications. She has recently been awarded a Future Leaders Fellowship to research advanced flow battery electrode materials. 


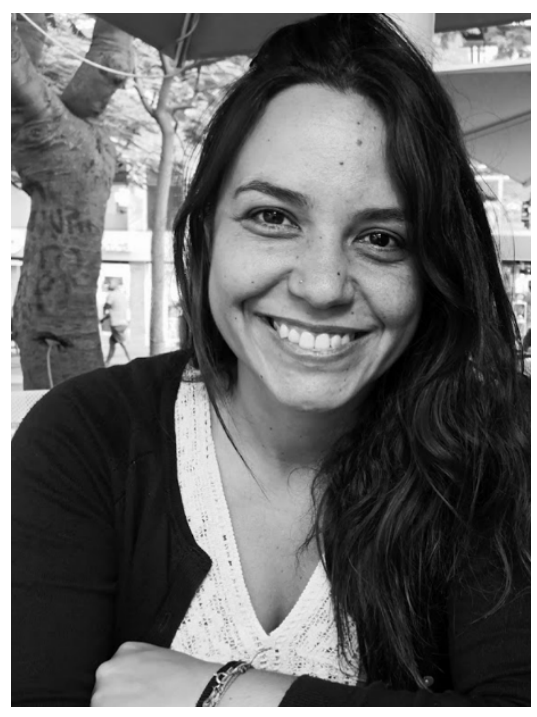

Ellsworth Bell received his BEng degree in Chemical Engineering from McGill University in 2014. After a stint in oil and gas, doing well evaluation work across the southern US, he joined Prof. Gostick's PMEAL research group at the University of Waterloo to pursue a master's degree. His research focuses on developing electrospun cathode materials to improve mass transfer in zinc - air flow batteries.

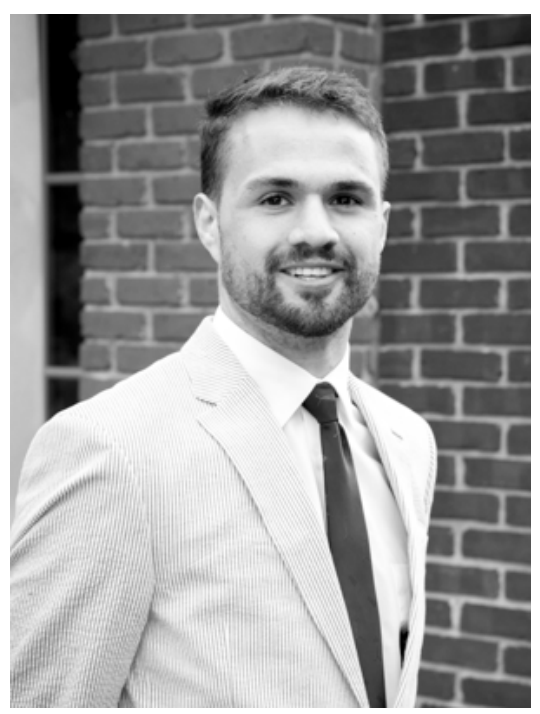

Jeff Gostick is an Associate Professor in Chemical Engineering at the University of Waterloo where he runs the Porous Materials Engineering \& Analysis Lab. His research is centred around understanding the structure-performance relationship in porous electrodes used in hydrogen fuel cell, redox flow systems, zinc-air cells, Li-ion batteries, and super-capacitors. His group uses a combination experimental characterization, novel production methods, and advanced custom computational tools. He is the lead developer of the open source pore network modelling project OpenPNM (openpnm.org), as well PoreSpy, a tool for porous media image analysis (porespy.org). Prof Gostick is a licensed professional engineer, has published over 70 journal articles, and was recently named an Emerging Leader by the Canadian Society for Chemical Engineering. 


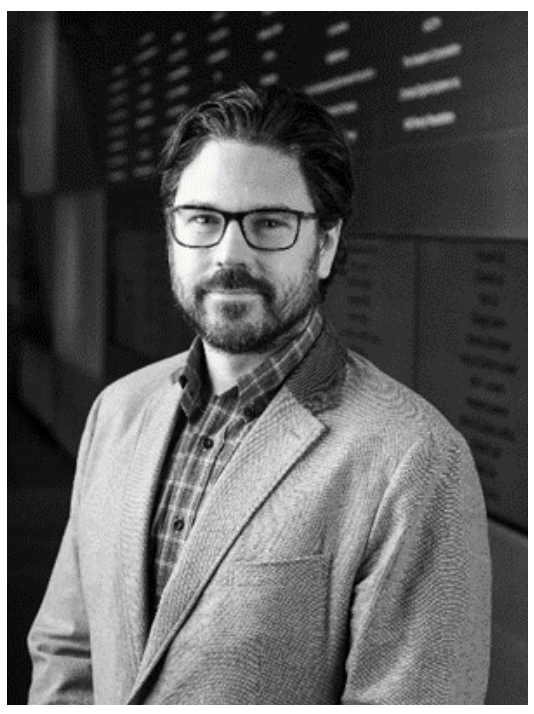

Servann Hérou graduated in chemical engineering at the Université de Technologie de Compiegne (France) and the Technische Universität Braunschweig (Germany) after obtaining his master thesis from the Max Planck Institute of Colloids and Interfaces (Germany). He then obtained his $\mathrm{PhD}$ in London at the Queen Mary University in the group of Magdalena Titirici where he developed lignin-based porous materials for supercapacitive applications. He is now a post-doc at Imperial College London working on hard-carbon anodes for sodium-ion batteries. Besides music composition, he is passionate about the energy transition and how can humans make the world a better place for everyone.

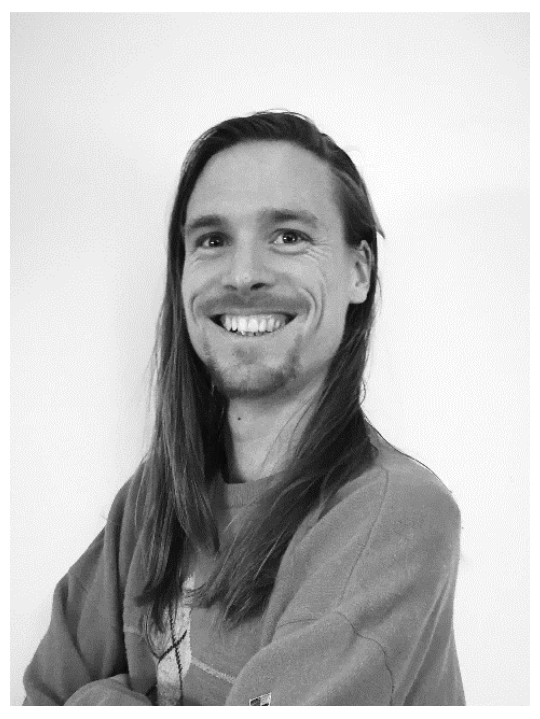

Philipp Schlee is a postdoctoral researcher in the Lignin Chemistry Group at Aalto University. Prior to joining Aalto University, he received a B.Sc. in Geosciences specializing in mineralogy and a M.Sc. in Materials Chemistry at the University Bremen in 2014 and 2016, respectively. He obtained his $\mathrm{Ph} . \mathrm{D}$. from Imperial College London in 2020 where he worked on electrospun lignin-based carbon nanofibres for energy storage applications. His recent research interests focus on the development of wood-derived lignin-carbohydrate precursors for micro/nanostructured carbon materials for energy storage applications and bio-composites. 


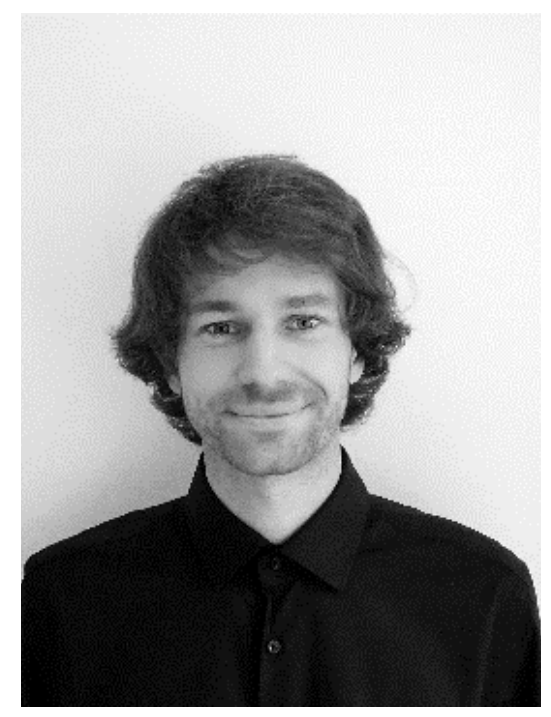

Magda Titirici has a Ph.D. from University of Dortmund (2005). She then did a post-doc and become later a group leader at the Max-Planck Institute of Colloids and Interface. She worked at Queen Mary University of London between 2013-2019 as a Reader and Full Professor. She is currently a Chair in Sustainable Energy Materials at Imperial College London and a RAEng Chair in Emerging Technologies. Magda has over 250 highly cited publications in the field of sustainable materials for energy storage and conversion and serves as an associate editor for $\mathrm{J}$. Mater. Chem. A and Materials Advances with RSC. She also serves as the president elect for the RSC materials chemistry division.

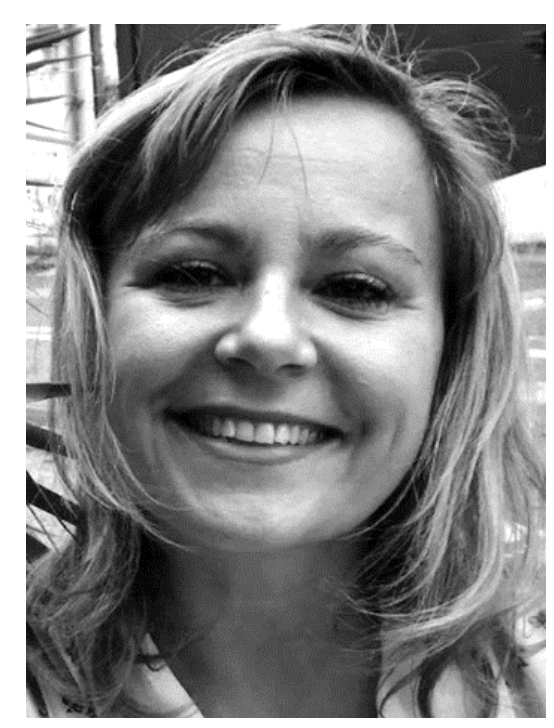

Dan Brett is Professor of Electrochemical Engineering at UCL and co-director of the Electrochemical innovation Lab. His research interests are in developing the materials, devices and diagnostic tools to help realise the 'age of electrochemical power'. Dan is an academic founder and member of the Faraday Institution Expert Panel, Director of Innovation at Bramble Energy and an Executive Director of Prosemino. 


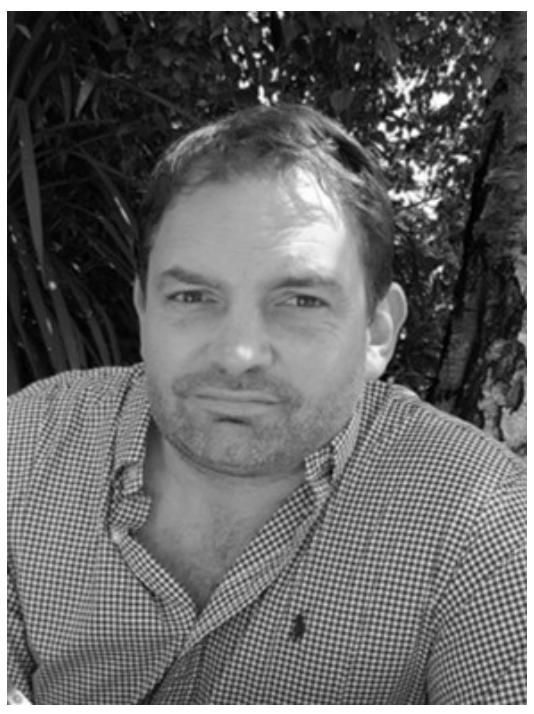

Paul Shearing is a Professor in Chemical Engineering at University College London where he holds The Royal Academy of Engineering Chair in Emerging Battery Technologies. His research interests cover a broad range of electrochemical engineering themes with a particular interest in the relationship between performance and microstructure for energy materials.

He co-directs UCL's Electrochemical Innovation lab and leads the UK STFC Global Challenge Network in Batteries and Electrochemical Devices. He was a founding investigator of the UK's Faraday Institution, where he chairs the Training \& Diversity Panel and is PI of the LiSTAR programme investigating Li-Sulfur battery technologies.

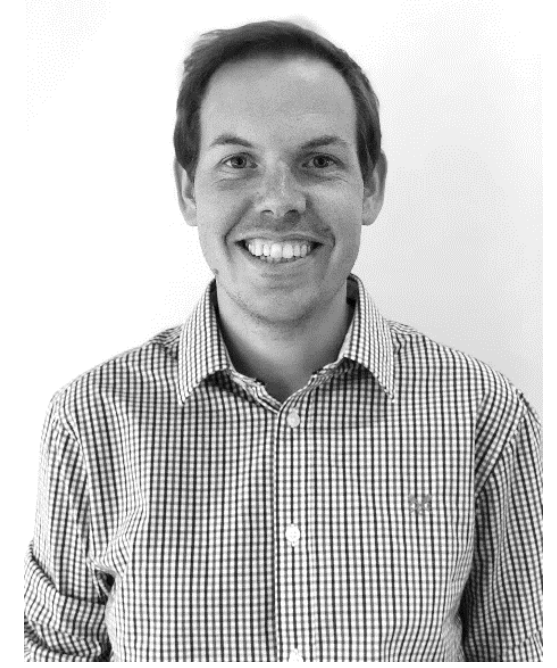

Rhodri Jervis graduated from Balliol College, Oxford in Chemistry in 2008 and after a short time working for a medical research spinout in his hometown of Swansea obtained his PhD in fuel cell catalysis from University College London in 2015. After a post-doctoral research position in redox flow batteries he took up a position as lecturer in Chemical Engineering in UCL in 2018. He is currently the project lead for the Faraday Institution fast-start on Li ion battery degradation. His research interests focus on studying energy materials using a combination of electrochemical and x-ray techniques. 


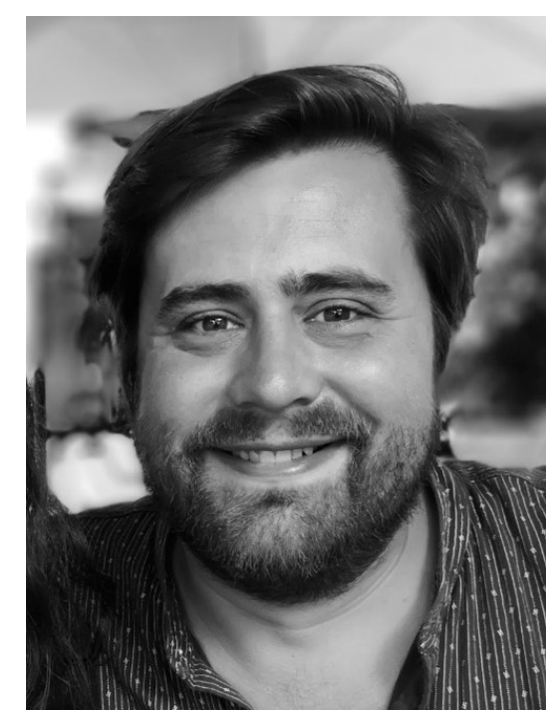

\section{Introduction}

Electrospinning is a highly versatile technique for generating nano-fibrous materials for a wide variety of applications. Using a large bias voltage, a wide range of polymers can be drawn out and 'spun' onto a collector, producing fibres with diameters less than $1 \mu \mathrm{m}$. Though the process of electrospinning can be traced back to patents from the early 1900s [1] and was theoretically described in the 1960s by Geoffrey Taylor [2], it is in recent years that the ability to produce controllable nanofibers has attracted increased interest in the area of nanotechnology, with widespread use in the areas of filtration [3], membranes for water treatment [4] and textiles [5]. In particular, the similarity of the structures produced via electrospinning to the fibrous extracellular matrices of human tissue has meant that the field of medicine has been one of the earliest and most extensive adopters of the technique [6], finding application in the areas of tissue engineering [7], regenerative dentistry [8] and drug delivery [9].

A major area where fibrous porous media plays a significant role is in renewable energy, and in particular electrochemical energy storage and conversion devices. With increasing focus on electrification of industrial, domestic and transport sectors using renewables as a route to decarbonisation, commercialisation of electrochemical devices such as batteries and fuel cells is vital, and there is increasing recognition of the need for advanced methods of electrochemical engineering [10]. In order to operate, electrochemical devices normally require a so-called 'triple-phase boundary', a nexus of electron transfer, reactant mass transport and electrolyte, to exist that allows the electrochemical reaction to occur. For this reason, carbonaceous porous media are ideal materials for many key structures in these devices, allowing good conductivity of electrons as well as high porosity, leading to effective transport of gaseous or solution-phase 
reactants to the surface of the structures. As well as optimising these properties, modification of the surface of carbons via heteroatom dopants or nanoparticles can assist in catalysing many electrochemical reactions in order to improve their performance. Therefore, in recent years, electrospinning has been highlighted as a key technology for electrochemical energy storage and conversion devices as a way to produce optimised microstructures to control mass transport and surface reactivity.

In this review, a brief methodology of the electrospinning process, including best practice principles, is given before focussing on four key technologies where conductive fibrous media are employed: redox flow batteries, low-temperature fuel cells, metal-air batteries and supercapacitors. Though there are many other technologies where electrospinning can play a role, including solar cells [11] and solid oxide fuel cells [12], the scope of this review is limited to carbon-based fibres for low-temperature devices for which mass transport is vital (flow batteries, fuel cells and metal-air batteries), as well as a recent perspective on the growing interest in the use of biomass sources for electrospun fibres for supercapacitors. Specifically, we do not cover electrospinning as a route to electrode materials that involve dimensional change; for example, with intercalation reactions in lithium-ion batteries, and the reader is directed to the recent reviews on the use of electrospinning in secondary batteries from Jung et al. [13], alkali metal batteries from Ilango and Peng [14] and from Santangelo that has an extensive section on lithium-ion batteries [15]. In addition, for information on the use of electrospinning for lithium-sulfur batteries, the reader is directed to the recent comprehensive review focused solely on this technology from Liu et al. [16] and for a very recent and comprehensive summary of the use of electrospinning in sodium ion batteries, to the review from Wang et al [17]. Therefore, devices that benefit from the tuneable microstructure of carbon fibres whereupon surface redox processes take place are covered within. 


\section{Methodology}

\subsection{Configuration}

In its purest form, any electrospinning set-up requires three major components: i) some method of feeding the spin-dope (polymer-containing solution) and charging it (usually called the spinneret), ii) a high voltage power supply to create the large potential field required, and iii) a collector of some kind to collect the electrospun fibres ejected from the feed. The spin dope feed and spinneret charging creates an elongated droplet at the tip of the needle called a Taylor cone, and the large electric field between the spinneret and the collector draws the polymer out, spinning it into small fibres on the collector (Fig. 1). One of the spinneret or the collector is charged (either positively or negatively) and the opposite component is grounded using the power supply. What follows is a step-by-step description of the different variations of these components that are commonly used and what advantages or features they provide.

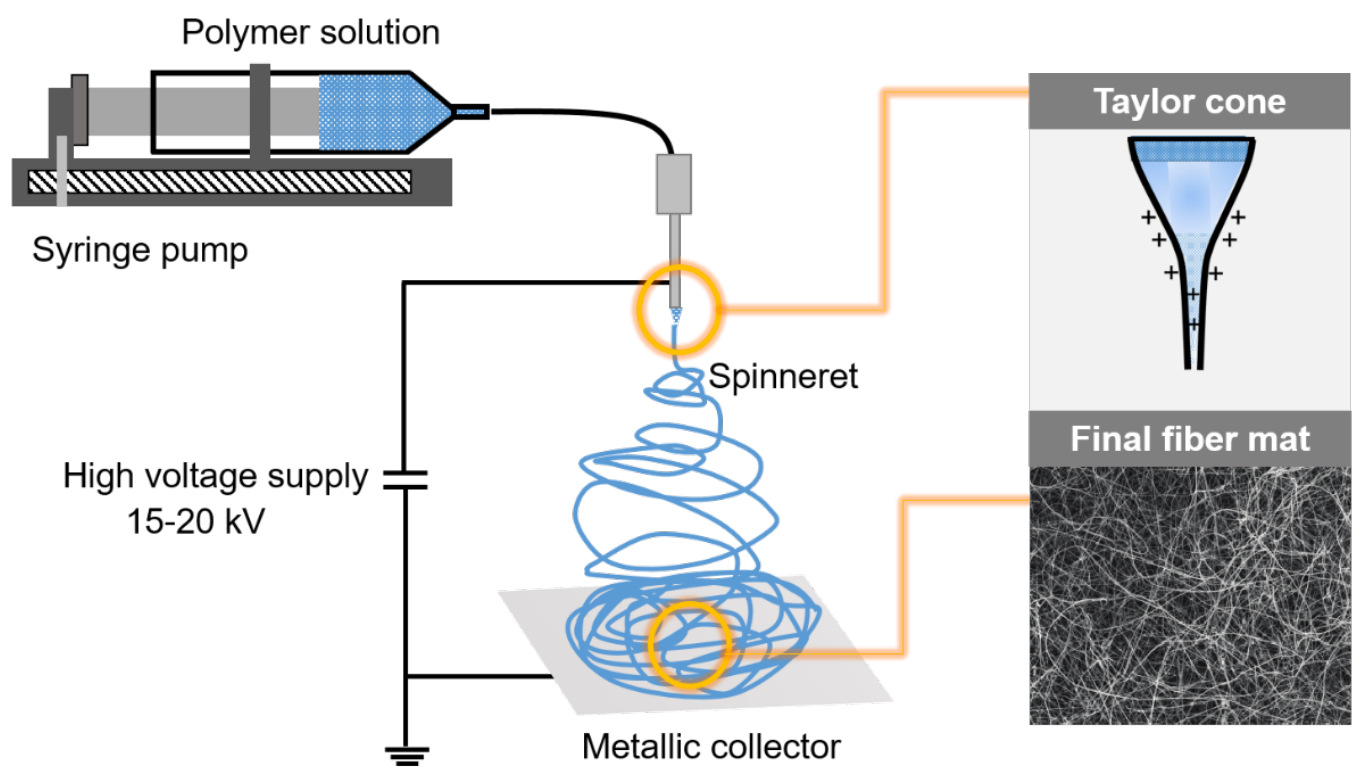

Fig. 1. Typical lab-scale electrospinning set-up. This set-up uses a needle and a flat horizontal metallic collector. Other configurations are also possible, as detailed in the main text.

\section{$2.2 \quad$ Feed}

The simplest way to feed an electrospinning process is with a syringe and a syringe pump. Even simple syringe pumps have a high degree of control of the flow rate, making them good candidates for electrospinning. Attaching a metal needle to the end of the syringe, and charging (or grounding) the needle fulfils the basic feed requirements for electrospinning and is often as far as researchers need to go for proof-of-concept studies [18-23], or basic morphology work [24-28]. An extension of this method is to utilize multiple needles on the same syringe pump 
to increase the material throughput and the electrospinning footprint on the collector [29-31]. This improves the deposition rate of the fibres (which can be prohibitively low for industrial scale-up when using a single spinneret) and can be a route to increased production rates.

Co-axial electrospinning is a similar method, but rather than one needle there are two arranged as a core (central needle) and shell (annulus). When used with different spin-dopes in the core and shell, co-axial electrospinning can produce composite materials [32-36], hollow materials [37-39], flat ribbon fibres [40-42], or even be used to modify fibre thickness [43,44]. This method can also be used to spin materials that could not normally be electrospun due to high viscosity [45] or low dielectric constant [46]. Similarly, systems with more than one shell (3+ different polymer solutions) have been used to create multilevel structures including embedded nano-wires $[47,48]$.

Another common method is to heat the spinneret and spin-dope to electrospin at elevated temperatures. This can be carried out for a variety of reasons. In melt electrospinning, the polymer is a solid at room temperature and needs to be heated in order to be effectively processed. This can be achieved by temporarily melting the polymer with laser-induced heating [49-52], using a hot jet of gas in the shell of a coaxial device [53,54], or heating the entire spinneret with a circulating heating fluid $[55,56]$. Similarly, these methods can be used for spinning polymers that need to remain at high temperatures throughout the electrospinning process [57-59], or as a means of affecting fibre morphology [60,61]. Local laser heating has also been used in-situ to heat the Taylor cone [62] or directly on the deposited fibres to alter their morphology $[63,64]$.

Other methods do not use a traditional spinneret; in free surface electrospinning, a surface is coated in a thin layer of spin-dope, often by submerging it in a solution bath and then rotating it near the counter electrode. Most commonly, this is done with a spinning wire electrode [6568] but similar results can be achieved using a rotating drum electrode [68], a splash electrode [69] and a spiral coil electrode [69]. Needle-disk electrospinning [70], or profile multi-pin electrospinning [71] coat surfaces that have been profiled, normally with pins, to control the size and location of Taylor cones. Other free surface methods will simply utilize a reservoir of spin-dope [72,73] or a series of reservoirs arranged as a pyramid [74,75]. The main difference with free surface electrospinning is, as the name implies, rather than a single Taylor cone forming in a fixed location, multiple Taylor cones form freely across the surface. These 
methods are particularly useful for scaling up the electrospinning process, as making any commercially relevant material with a single spinneret is incredibly time-consuming.

The electrospinning process is highly random and achieving uniform thickness across the material can be challenging. A commonly used method to achieve this is to raster the needle back and forth to allow the fibres to deposit uniformly over the collector. The entire feed assembly can be rastered. However, it may be easier to simply move the needle and connect the syringe pump to the needle through flexible plastic tubing. Additionally, approaches such as near field electrospinning [76,77] and magnetic alignment [78-80] can allow for greater control of fibre morphology and orientation, though they are out of the scope of this review.

\subsection{Field potential}

The main driving force in the electrospinning process is the large potential across the spinneret and the collector. Generally, for any given polymer-solvent system there is a minimum required field voltage $\left(\mathrm{kV} \cdot \mathrm{cm}^{-1}\right)$, defined by both the potential of the power supply and the operating distance between the spinneret and the collector, below which the potential field is not 'pulling' enough to draw out electrospun fibres [81,82]. On any given electrospinning system, this will need to be tuned locally, as it will be dependent on relative humidity, electrode-counter electrode distance [83] as well as solution concentration [84], viscosity [85] and flow rate [86]. The following progression is generally true for operating at field potential $\sigma$ in a system with $\sigma_{\mathrm{MIN}}$ minimum field voltage.

For $\sigma \ll \sigma_{\mathrm{MIN}}$, no electrospinning will take place. As $\sigma \rightarrow \sigma_{\mathrm{MIN}}$ the system will start to occasionally deposit very large fibres onto the collector. The fibres will be wet with solvent and likely damage any pre-existing fibres, as well as lacking any control in fibre size. When $\sigma$ $=\sigma_{\mathrm{MIN}}$ the system will continually deposit fibres onto the collector. If the flow rate of the spindope is not properly tuned the system will 'sputter,' causing unequal deposits of fibre and solution onto the collector, often damaging the sample. Electrospinning at these lower field potentials leads to bead-like morphology and often inconsistent operation. As the field potential increases the fibres will first become more consistent in size and morphology (more fibres, fewer beads) and the fibre size will decrease as the field potential increases. Eventually, at $\sigma_{\mathrm{MAX}} \gg \sigma_{\mathrm{MIN}}$, the system will stop making fibres and instead electrospray nano- and microbeads of polymer at the collector [87]. Much like the minimum field voltage, the maximum field voltage is highly dependent on the individual electrospinning system. 
The polarity of the applied potential field can also affect the electrospinning process. As with the applied voltage, described above, there is no correct polarity; rather, it is highly dependent on the individual process. Studies have been undertaken to dichotomize the effects of different polarities, usually focusing on one specific polymer-solution system. Wu et al. found that electrospinning aqueous polyvinyl acetate while negatively charging the collector or positively charging the spinneret outperformed the other configurations when considering consistent fibre production and smaller fibre diameter [88], which was confirmed by Kilic et al. for a similar system of polyvinyl in alcohol and water [89]. Ali et al. found that charging (negatively or positively) the spinneret resulted in better control of polyacrylonitrile-dimethyl formamide (PAN-DMF) systems [90], a result later confirmed by Kok and Gostick [91]. Urbanek found that when spinning polycapralactone/chitosan in hexafluoropropanol, negatively charging the spinneret resulted in greater ease and efficiency in a desired surface modification [92]. The opposite was found by Stachewiscz et al. [93], who determined that the desired surface functionality in their nylon-acetic/formic acid electrospun materials was better served using a positive power supply. There is, as yet, no clear underlying reason for the observed differences in consistency of fibres obtained with different polarities and the literature suggests that it is specific to particular combinations of polymers and polarities, though it is an interesting avenue of investigation.

Typically, an electrospinning setup will use one of the configurations described above; one high voltage direct current (DC) power supply that charges either the spinneret or the collector, with the other grounded. Other configurations are used but are far less common. Alternating current (AC) power supplies have been used to electrospin super-hydrophobic surfaces [94], highly-aligned nanofibres [95], as well as micro helical polymeric structures [96]. Tong and Wang created a novel electrospinning set-up that used alternating positive and negative DC power supplies to greatly increase how thick the deposited electrospun mat could be [97].

\subsection{Collector}

The final component for electrospinning is the collector. Fibre morphology is usually dependent on other 'up-stream' properties, such as concentration, working distance and voltage, as previously outlined, while the collector largely contributes to the overall material architecture. The simplest method of collection is to ground (or charge) a flat piece of metal. Referred to as a flat plate collector, this method provides no additional benefits other than collecting deposited fibres. Flat plate collectors, much like single spinnerets, are often used for proof-of-concept or morphological studies. They are excellent for demonstrating something 
can be electrospun but offer no real avenues for scaled-up production. Flat plate collectors can also be modified to generate more complex or interesting materials. Common modifications include patterning the surface with channels [98,99] or hole arrays [100]. Patterning the collector in this way controls where and how the fibres deposit during the process.

One level of complexity above the flat plate collector is the rotating drum collector. In this case, the collection of fibres is carried out on a grounded (or charged) metallic drum that is rotating. A major benefit of this collector is that it allows for creation of larger samples with more consistent properties. As electrospinning typically releases a conical zone of deposited fibres, there can be differences in the amount of material deposited based on location (i.e. radial position). By rotating the drum there will be less variation as the collector is effectively moving. Combining a rotating drum collector with a rastered spinneret will achieve this in all directions while also increasing the sample size. Rotating drum collectors can also impart a large amount of anisotropy into the materials. If the surface velocity of the drum is fast compared to the velocity of the fibre deposition, the fibres will align around the circumference of the drum. Similar results can be obtained by using a rotating wire collector, made up of a 'drum' of individual wires [101]. In this mode, the fibre anisotropy is less dependent on the velocity of the collector and more an intrinsic characteristic of its geometry. Rotating drum collectors can also be used to induce morphological gradients in the electrospun materials. In cone electrospinning, the collector is a rotating cone, making the tip to collector distance vary along the length of the collector [102].

A third class of collectors is needle, tip or pin collectors. These collectors are usually comprised of arrays of patterned pins. During electrospinning the fibres will preferentially deposit on the pins making highly structured materials [103]. Single pin collectors can also be utilized. Chang et al. used a rotating needle to make micro-ropes [104] and Rafique et al. have used a needle to make highly aligned fibres [105], while Yu used a charged nail to make electrospun fibres with 'closed-loop' (continuous) structures [106].

Many other varied collectors are possible, and they can be customized depending on need. Collectors have been made from soft lithography of silicon substrates [107] and 3D printing of delicate designs [108]. Rotating ring collectors have been used to generate and collect nanofibre yarns [109] and highly porous 3D scaffolds have been generated by electrospinning onto a helical spring [110]. As the collector needs only act as a counter-electrode and target, there are nearly infinite different styles and types that can be utilized. 


\subsection{Best practices}

The focus of this review is electrospinning for electrochemical devices. For this section, polyacrylonitrile fibres (PAN) dissolved in dimethylformamide (DMF) will be used as a model system; while many of the methods described above can be used with a variety of polymers to produce useful electrochemical materials, PAN/DMF electrospinning is the most common as PAN is a high-yield carbon precursor, which is often a requirement in electrochemical devices. However, DMF is toxic and care should be taken when working with this polymer/solvent combination. What follows is a set of best practices for electrospinning PAN/DMF from a simple single spinneret and a rotating drum or flat plate collector. Additionally, electrospinning is generally conducted at room temperature (though elevated temperatures of the polymer solutions are often used, as discussed in section 2.2) and at low relative humidity to increase the dielectric constant of the air gap between the spinneret and the collector, and so the following discussion also assumes these conditions. There is a wide variety of approaches taken in the literature, however, and varying these parameters can have an effect on fibre morphology and consistency.

Best results (in terms of ease of electrospinning) are achieved by grounding the spinneret and charging the collector with a negative voltage power supply. The first parameter choice to make in a system is the electrospinning distance. The best value will depend on the specific setup but typically $12-15 \mathrm{~cm}$ is an appropriate tip-to-collector distance.

A solution is prepared by mixing PAN (MW 150,000 Da) in anhydrous DMF and stirring without heating for more than 12 hours, normally overnight. The concentration of the spin dope depends on the desired fibre size. Typically, on the lower end will be $9-10 \mathrm{wt} \%$, which will produce fibres that are $<500 \mathrm{~nm}$. The highest concentration of PAN that can be electrospun without heating or other modifications is $13 \mathrm{wt} \%$, which will produce fibres that are larger than $1 \mu \mathrm{m}$ in diameter. Generally, $12 \mathrm{wt} \%$ leads to facile electrospinning and consistent fibres between $800 \mathrm{~nm}$ and $1.5 \mu \mathrm{m}$.

A syringe should be filled as gently as possible with spin-dope, avoiding bubbles, especially small bubbles as they will affect the flow of the material and are very hard to remove due to the highly viscous solution. The flow rates are very low, so only a small amount ( $5 \mathrm{~mL})$ needs to be added, especially initially. A 14 - 18 gauge (1.628 - $1.024 \mathrm{~mm}$ inner diameter) needle should be attached to the syringe and loaded into a syringe pump. The needle of choice is determined by the viscosity of the solution. Generally, 16 gauge needles are effective but bigger or smaller needles may be necessary for more or less viscous spin-dopes. 
To find the correct flow rate, the flow should be increased slowly at small increments. Using a non-friable wipe, the end of the needle is cleaned - if a 'bulb' of spin-dope does not immediately replace it, the flow should be increased. If the 'bulb' is quickly replaced and then very quickly starts to fall off the needle, the flow is too high. The flow rate for the conditions listed above is typically between 0.8 and $1.0 \mathrm{~mL} \cdot \mathrm{hr}^{-1}$.

The final step is to connect the ground to the spinneret and turn on the power supply. The power is gradually increased until the Taylor cone forms and becomes stable. If the spin-dope is periodically dripping, the voltage is insufficient; if the Taylor cone is growing smaller as the 'bulb' is consumed and then reforms as the flow replaces it, the voltage is too high. During the process of electrospinning, as more material is deposited and the thickness of the (insulating) fibre mat increases, it may be necessary to increase the operating voltage to account for the change in dielectric constant over the spinning distance in order to avoid inhomogeneities through the thickness of the electrode [111]. Periodically, while spinning, the tip of the spinneret will need to be cleaned as excess fibre and spin-dope build-up can affect the electrospinning performance. This is easier using a non-conductive stick with a tiny amount of non-friable paper wrapped around the end (KimWipes).

Following electrospinning, it is usually desirable to carbonise or graphitise the mats to make a conductive material. Usually, best results are achieved using a tube furnace and following the following general steps, though methodology does vary in the literature and may need to be adapted to different polymer precursors. In general, however, higher temperatures and carbonisation times will lead to higher degrees of graphitisation, but often at the cost of reduction in surface functionality of the materials. First, if there is a desired thickness for the final material, the mats can be placed between alumina plates, with the space controlled by spacers. The plates will ensure the final product is flat and with consistent thickness throughout. The sample should then be placed in a tube furnace and heated at a rate of $5^{\circ} \mathrm{C} \cdot \mathrm{min}^{-1}$ until $250{ }^{\circ} \mathrm{C}$. The sample should then be maintained at this temperature, in air, for at least 75 minutes. This is in order to allow for cleaving of the triple bonded $\mathrm{N}$ and a dehydrogenation reaction to occur, requiring oxygen, before the graphitisation occurs. Towards the end of this rest period, nitrogen or another inert gas should be used to provide an inert atmosphere in the furnace, after which the temperature is again increased at a rate of $5^{\circ} \mathrm{C} \cdot \mathrm{min}^{-1}$. For low-temperature furnaces, the temperature should remain at $850{ }^{\circ} \mathrm{C}$ and $1150{ }^{\circ} \mathrm{C}$ for 40 minutes each. For higher temperature processes, a similar plateau every $300-350{ }^{\circ} \mathrm{C}$, for example at $1500{ }^{\circ} \mathrm{C}$ and 
$1800{ }^{\circ} \mathrm{C}$, can be applied. After the final temperature plateau, the temperature is again reduced at a rate of $5^{\circ} \mathrm{C} \cdot \mathrm{min}^{-1}$, until $250{ }^{\circ} \mathrm{C}$, and then allowed to cool to room temperature.

These general principles should produce repeatable and reliable results for PAN-based fibres for the electrochemical energy storage devices discussed in the remainder of this review. However, a key advantage of electrospinning is in its adaptable nature and use of any polymers; many interesting variations of these basic methods will be presented in this review as a route to advanced functional materials.

\section{Redox flow batteries}

\subsection{Overview the historical development of electrospun materials in redox flow batteries}

Compared with other energy storage technologies, redox flow batteries (RFBs) have a unique ability to decouple the energy density and power output. This is due to the operating principle of RFBs, whereby electrolytes containing the active species, stored in the external tanks, flow into a conversion device, or 'stack', where it is charged or discharged. In this way, the capacity of the battery can be increased simply by increasing the electrolyte inventory in the tanks, and the power of the battery by increasing the size of the stack. Therefore, it is one of the leading candidates for fulfilling the multi-scale requirement of optimal energy storage for the electricity grid. Among diversified chemical couples, the all-vanadium redox flow battery (VRFB) has attracted the most commercial attention due to its resistance to crossover effects from electrochemical-recoverability of the electrolytes due to its single-element chemistry [112]. During discharging, a negative electrolyte (anolyte) and a positive electrolyte (catholyte) are pumped through porous, conductive electrodes, where the electrodes serve as a platform to initiate redox reactions on their surface with no dimensional or chemical change of the electrode. For this reason, carbon felt or paper composed of graphitised carbon fibres in the order of $10 \mu \mathrm{m}$ diameter are often employed in RFBs, providing porous, conductive electrodes with a high surface area that are chemically and mechanically robust in the operating regime of RFBs.

Though the VRFB is the most developed chemistry, it has not been extensively commercialised because of its sluggish kinetics on the current electrode materials [113]. The slow charge transfer rate leads to an excessive charge transfer overpotential, risking competing side reactions such as the hydrogen evolution reaction (HER, on the negative electrode) and diminished electrolyte utilization. Generally, a large stack size is required to satisfy high power 
output demands with commercial electrodes, which results in a high upfront cost of the system. Additionally, when operating at higher current densities or at lower states-of-charge (where the concentration of the active species in the electrolyte is diminished), there are significant mass transport overpotentials that reduce the voltage efficiency of the battery. To operate under high current without increasing polarization and depleting cell efficiency, enlarging the reactive surface area of the electrode material and tuning the microstructure to improve mass transport are two approaches that can improve performance without requiring improvements to electrochemical activity (e.g., via the use of catalysts or alternative chemistries). Decreasing the fibre diameter in these electrode materials can improve the performance of the RFBs via an increase in the specific surface area, and controlling the size, amount and alignment of pores can help to optimise the microstructure for more effective operation in the mass-transport limited regime. Additionally, modifying the fibre surface properties to introduce surface functional groups and mesoporosity can help catalyse the reactions and improve the wettability of the electrodes, lowering the activation overpotentials in these systems. Electrospinning has recently emerged as a flexible technique that can address all these aspects of performance enhancement in RFBs, allowing the synthesis of free-standing mats of small, high surface area fibres, control of microstructure through the operating parameters of the spinning process and control of surface functionality by the ability to spin many different polymers and additives of varying compositions.

The development of electrospun materials for VRFBs was first demonstrated in 2013 [114,115] and the historical development of the usage of electrospun materials in RFBs is outlined in Fig. 2. Due to the electrical conductivity requirements for use as the electrode material, carbonisation conditions were firstly taken into consideration to achieve high conductivity without compromising the advantage of achieving high surface area. The first two papers $[114,115]$ investigated electrospun materials as positive electrodes for $\mathrm{VO}_{2}{ }^{+} / \mathrm{VO}^{2+}$. Wei et al. [114] analysed conductivity and catalytic activity of electrospun carbon nanofibres (ECNF) carbonized at different temperatures. As desired, ECNF were observed with fibre sizes two orders of magnitude smaller (100 - $200 \mathrm{~nm}$ diameter) than commercial electrodes (10 - $15 \mu \mathrm{m})$, with a smooth surface and a turbostratic carbon structure. Their temperature study showed that ECNF carbonized at $1,000^{\circ} \mathrm{C}(\mathrm{ECNF}-1000)$ demonstrated the highest conductivity of 8.54 $\mathrm{S} \cdot \mathrm{cm}^{-1}$ compared to barely $0.138 \mathrm{~S} \cdot \mathrm{cm}^{-1}$ at $700{ }^{\circ} \mathrm{C}$ carbonisation. In general, it can be assumed that conductivity results in the literature correspond to through plane conductivity where the majority of the electron transport to the current collector takes place, and unless stated 
otherwise in this review, conductivity refers to this direction. Comparing to a charge transfer resistance of $57.2 \Omega \cdot \mathrm{cm}^{-2}$ for commercial carbon felt (CF), ECNF-1000 reduced the resistance to $22.4 \Omega \cdot \mathrm{cm}^{-2}$ at $1 \mathrm{~V}$ polarization voltage. It was shown that ECNF-1000 benefited from its higher carbon content, graphitisation degree, larger surface area and unique carbon structure [114]. Low resolution- and high resolution-transmission electron spectroscopy (LRTEM and HRTEM) characterization shown in Fig.3( $a$ and $b$ ) demonstrate the particular internal structure of the carbon for ECNF-1000, where the elongated graphitic crystallites aggregated and aligned along the fibre axis. Their paper stated this was forced by the rotating alignment effect during electrospinning [114]. Another pioneering work by Flox et al. [115] also analysed the different collectors' effects on ECNF. In Fig.3(e and f), the low resolution- and high resolution- scanning electron spectroscopy (LRSEM and HRSEM) images show fibre alignment induced by using a rotating collector, and is compared to unaligned fibres generated on a plate collector shown in Fig.3(c and d). Aligned fibres had larger and less variation of fibre diameter than unaligned fibres. Alignment also sharply enhanced ECNF conductivity, especially in its aligned direction, which was four times higher than the unaligned material, and six times higher than commercial felt. Wei et al. [116] also investigated a higher temperature window from $1,000^{\circ} \mathrm{C}$ to $1,500^{\circ} \mathrm{C}$, and found $1,000^{\circ} \mathrm{C}$ worked best for the $\mathrm{VO}_{2}{ }^{+} / \mathrm{VO}^{2+}$ redox couple. Increasing the temperature led to functional group depletion even though higher temperature increased the number of graphite crystallites' edge planes, therefore, an optimized temperature was reported, balancing those two enhancement factors. The lateral graphite crystallite size (La) obtained from Raman spectroscopy increased greatly from $1.4 \mathrm{~nm}^{-1}$ to $2.3 \mathrm{~nm}^{-1}$ with carbonization temperature raising from $1,000{ }^{\circ} \mathrm{C}$ to $1,500{ }^{\circ} \mathrm{C}$, which confirmed the improved degree of order (graphitisation degree) with temperature. However, the consumption of $\mathrm{N}$ and $\mathrm{O}$ content with higher temperature inevitably caused reduction of functional groups. Xu et al. [117] discovered that $1,100{ }^{\circ} \mathrm{C}$ exhibits the highest activity for the negative $\mathrm{V}^{2+} / \mathrm{V}^{3+}$ redox couple and tested ECNF as an ultrathin free-standing electrode achieving $74.1 \%$ energy efficiency (EE) at 40 $\mathrm{mA} \cdot \mathrm{cm}^{-2}$. Aside from carbonisation temperature studies, various carbonisation times $(30,60$, 90 and $120 \mathrm{~min}$ ) were 


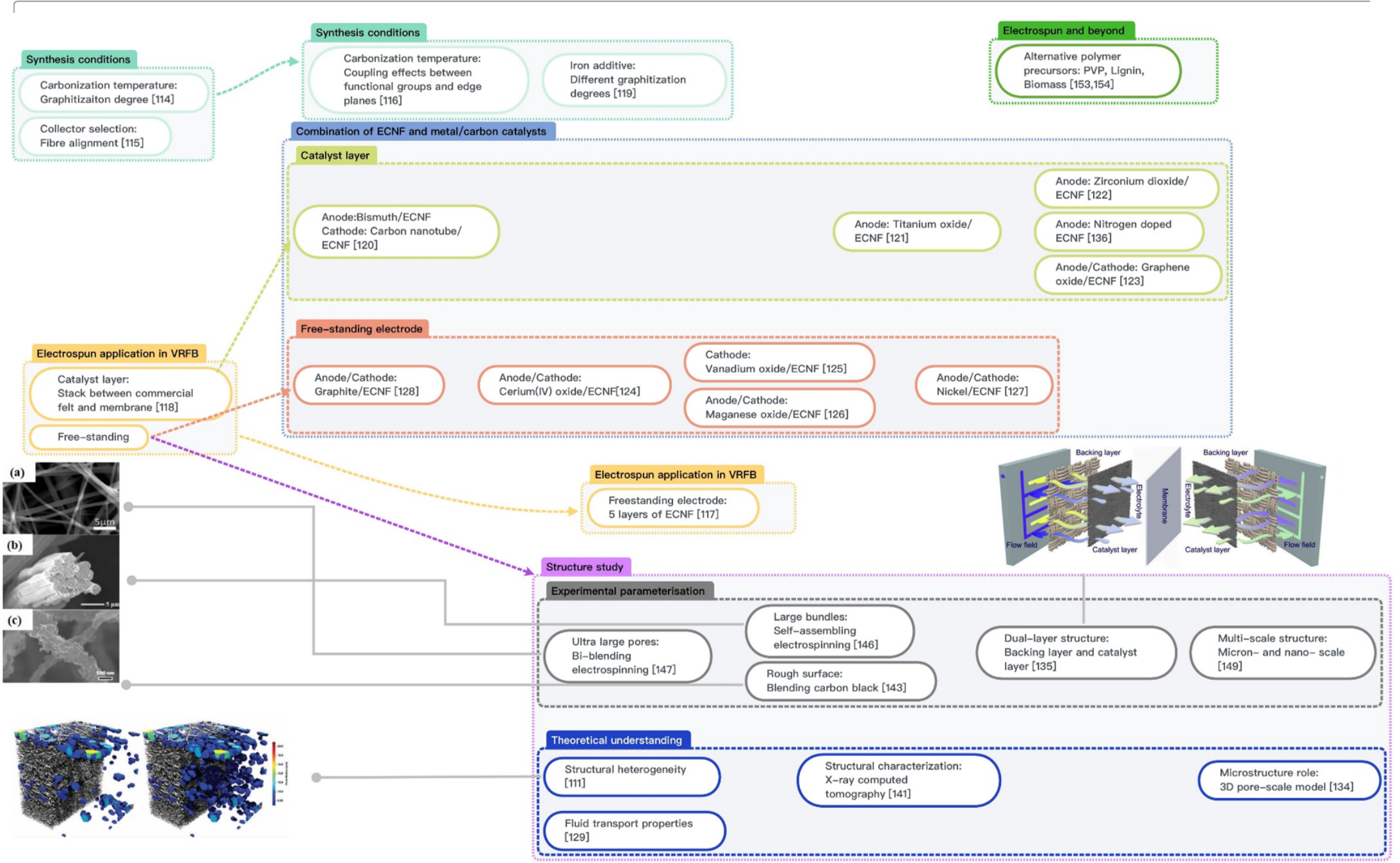

Fig. 2. Historical development of electrospinning in RFBs since 2013, grouped into studies of similar aspects of electrospinning: the effect of synthesis conditions (cyan), combination with catalysts (light blue), application in VRFB (yellow), microstructural studies (pink) and the use of polymers other than PAN (green). Reproduced from Ref. [111, 143] with permission from John Wiley and Sons, and from Ref. [147] with permission from The Royal Society of Chemistry. 
investigated with 90 min delivering the best performance [118]. There was little variation in morphology and element composition, but strong influence on graphitisation degree with carbonisation time. The shape of the (002) diffraction peak from X-ray diffraction (XRD) patterns became sharper and its calculated crystallite size $\left(L_{\mathrm{C}}\right)$ increased from $1.0 \mathrm{~nm}$ to $2.0 \mathrm{~nm}$ with increased carbonisation time, indicating that building up carbonisation time improved the ordering of the turbostratic carbon structure. Various carbonisation time also led to different electrochemical activity for the vanadium couples. The electrical conductivity increased with graphitisation degree; however, the number of exposed catalytic edge-planes decreased with increasing graphitisation order. Therefore, a peak catalytic performance point was reached at 90 min carbonisation time. On the question of the impact of graphitisation degree, the authors designed experiments with an iron additive to achieve different graphitisation degree of ECNF [119]. The c-axis spacing $\left(d_{002}\right)$ from XRD pattern reduced to $0.340 \mathrm{~nm}$ and $L_{\mathrm{C}}$ of ECNF reached $8.6 \mathrm{~nm}$ at $1,300{ }^{\circ} \mathrm{C}$ with the catalysing activity of iron. Results also confirmed that there was a trade-off between increasing conductivity and decreasing catalytic activity on increasing graphitisation. The early-stage papers on synthesis conditions compared ECNF with commercial electrodes, and hinted at the potential for electrospinning to produce promising electrode materials for the VRFB with its remarkable enhancement of charge transfer and mass transport rate.
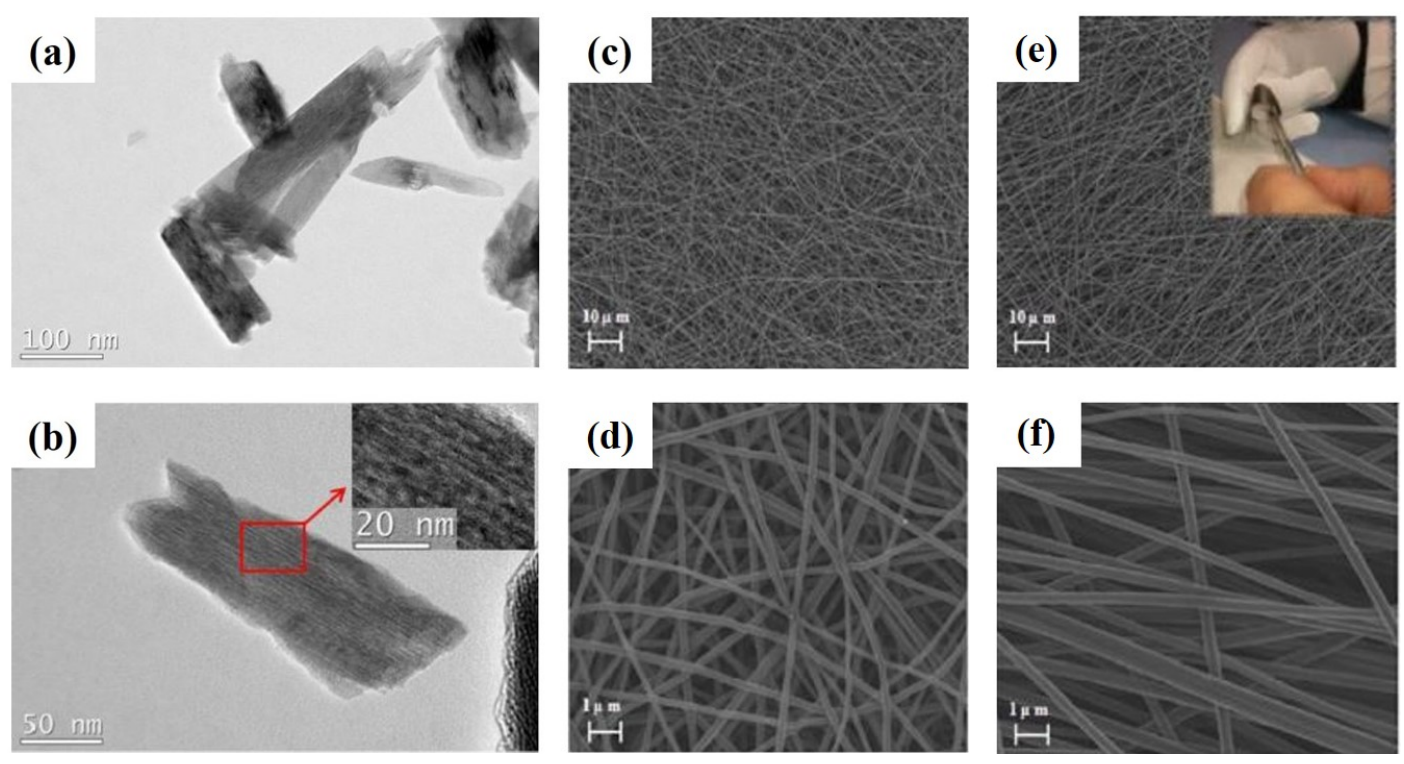

Fig.3. (a) LRTEM and (b) HRTEM of elongated graphitic crystallites in ECNF-1000 (inset: detail of the graphitic structure) [114]. (c) LRSEM and (d) HRSEM of unaligned ECNF. (e) LRSEM (inset: photograph displaying the flexibility of this material) and (f) HRSEM of aligned ECNF. Reproduced from Ref. [115] with permission from The Royal Society of Chemistry. 
Before the application of electrospun mats as free-standing electrode materials, electrospun fibres were initially coated or spun on the CF as a catalyst layer. When the polymer was directly spun on the commercial felt before carbonisation, there was a deterioration of commercial electrode performance. Researchers explained that this was due to the extra-introduced hydrophobicity with additional heat-treatment during graphitisation of electrospun fibres [118]. Therefore, electrospun fibres were generally separately prepared, then sandwiched between commercial felt and membrane layers [118,120,121]. Accompanied by the research on synthesis conditions, the joint functionality between the electrospun catalyst layer and metal catalysts has also been explored since 2015 [121-123]. The approach of mixing metal salt precursors into the polymer solution for electrospinning simplified two-steps of the metal crystallisation and carbon graphitisation into one. Another advantage is the metal nanoparticles were believed to have a more robust attachment to the carbon fibre support when co-spun with the polymer, preventing removal and loss of catalyst material during flowing of the electrolyte through the electrodes. Though this is inferred from scanning electron microscopy (SEM) images showing embedded particles in the fibres, the majority of papers do not provide direct proof of stronger adhesion. So far, a wide range of metals including Bi [120], Ce [124], V [125], $\mathrm{Mn}$ [126], Ti [121], Ni [127] and Zr [122], and carbon catalysts consisting of reduced graphene oxide (RGO) [123], graphite nanopowders [128] and carbon nanotubes [120]) has been studied and is outlined in more detail in Section 3.2. The formed electrospun/metal composites have been used as either a catalyst layer or free-standing electrode. When studying ECNF as a porous electrode, the microstructure and transport properties, including fibre size, pore size, porosity and permeability were found to play an important role. A model constructed by Kok et al. [129] for a hydrogen-bromine redox flow battery revealed that the fibre morphology had a large impact on the kinetics of the system, with smaller fibre size increasing the available surface for reaction. However, with a reduction in fibre size comes a reduction in permeability of the mats; on the reduction of the fibre size with a specified porosity (e.g. fibre diameter below $1.5 \mu \mathrm{m}$ with 0.9 porosity), mass transfer limitation starts dominating for the porous electrode. Although the specific surface area could be directly improved by decreasing fibre size, the effective surface area is more complicated relating to liquid transport properties. There were shown to be optimal fibre sizes for battery power output relating to different porosity values. Therefore, the model suggested an optimal structure, with fibre size of $1-2 \mu \mathrm{m}$ and porosity higher than 0.85 predicting the best battery performance. Since the fibre size of commercial felt is an order of magnitude larger, the electrospinning process was chosen to achieve this target structure. The Gostick group accomplished this through increasing the PAN concentration from $10 \mathrm{wt} \%$ 
to $12 \mathrm{wt} \%$ [130]. However, there was an unforeseen issue of lower limiting current with the designed electrospun structure, suspected to be due to structural heterogeneity and this conjecture was later verified through X-ray computed tomography (CT) combined with computational modelling of the flow properties of the electrode [111,131]. Subsequent work reduced the heterogeneity in the fibre size and in the structure through the thickness of the mat by employing a rotating drum collector [132]. Increasing the rotating speed of this collector also allowed a degree of alignment to be attributed to the fibres, instilling a directional anisotropy into the electrodes. The effect of this anisotropy was studied via the use of the lattice Boltzmann method (LBM) to model the fluid dynamics on the microstructures obtained from $\mathrm{CT}$ and compared to electrospun materials with random fibre alignment, as well as the commercial carbon felt (CF) [133]. The mass transport properties of the electrodes were found to be strongly correlated to the microstructure and an inverse relationship between permeability and mass transport was demonstrated, highlighting the ability of electrospinning to create tuneable microstructures with beneficial properties for RFBs. The combination of LBM with CT experimental data was recently applied to investigate the effect of microstructure on the mass transport, and to predict the electrolyte flow and reactant distributions of three different electrode materials (not electrospun) for non-aqueous RFBs by Zhang et al. [134]. Better electrochemical results with homogenous pores subsequently validated the close connection between the electrode microstructure and battery performance.

In summary, the development of ECNF for RFBs was driven by a desire to engineer electrodes with better charge transfer and mass transport properties. The tuneable microstructures, controllable surface-chemical compositions, and industrial scalability of ECNF make it attractive for the application as either a catalyst layer or a free-standing electrode for RFBs. The earliest attempts largely investigated the effect of the carbonisation step and discovered a potential for the use of ECNF in RFBs, showing an improvement of activity and mass transport properties via its surface chemistry and controlled morphology, which has inspired further 


\subsection{Catalysts additives and surface functional groups}

The application of electrospun mats as a catalyst layer was first explored by Wei et al. [118] and has been investigated in detail by Qixing et al. [135] using carbon paper, carbon cloth and graphite felt as the backing layer, providing mechanical support and accounting for the high mass transport resistance in the electrospun thin film applied to its surface. In this way, the ECNF provides a region of high surface area without impacting drastically on the higher permeability of commercial electrode materials with much larger fibre sizes. An outstanding performance of $80.2 \% \mathrm{EE}$ was achieved at a high current density of $240 \mathrm{~mA} \cdot \mathrm{cm}^{-2}$ with stability for more than 800 cycles. This catalyst layer also inspired the embellishing of the ECNF with metal catalysts, appearing in the literature two years after electrospun fibres were first demonstrated in VRFBs. The most common approach is to synthesise catalysts supported on electrospun fibres via blending the catalyst precursor into the polymer solution during electrospinning, followed by thermal treatment for simultaneous fibre carbonisation and catalyst crystallisation. Several papers have studied the catalytic activities and capabilities of different catalysts on improving the vanadium kinetics [120-127], using the voltages and separations of redox peak currents in cyclic voltammograms (CVs) as the performance metric, and the extracted ohmic, charge transfer, and mass transfer resistances from electrochemical impedance spectroscopy (EIS). The diverse application of electrospun material as either a freestanding electrode or a catalyst layer split the works of ECNF with catalysts composites into two groups.

Wei et al. [128] considered graphite nanopowders in the first instance to increase the concentration of edge-planes, correlating to their graphitisation degree studies [116,119], which was demonstrated as having catalytic activity for the vanadium redox couples. In this work [128], they considered electrospun fibres and graphite nanoplates as the electrode and electrocatalyst, respectively. The electrocatalytic activity was improved and increases with the amount of graphite nanopowders from $1 \mathrm{wt} \%$ to $2 \mathrm{wt} \%$ embedded in the matrix. The CV comparison in Fig. 4 (b) shows the graphite/ECNF composite having more than two times the redox peak current densities and more symmetric peaks than ECNF, which indicates more reversible kinetics on the graphite/ECNF composite surface. The low price of graphite powder compared to other carbon nanomaterials also makes it superior for large-scale applications. Despite not being emphasized in their work, there was an obvious morphology change imparted by adding graphite nanoplates, as shown in Fig. 4(a). While analysing the performance of 
increased reaction current densities or reduced charge transfer resistance, it is also pertinent to consider the impacts of morphology variations, particularly in the mass transport limited region of operation of RFBs (requiring tests in working flow cells). Further work by Wei et al. [120] separately added carbon nanotubes and bismuth to form composite catalyst layers. They found the catalysts embedded into electrospun fibres and crystallised near to the surface region (Fig. 4e), hypothesising that this results in a stronger adhesion of electrocatalysts to the fibres. The composite catalyst layers of bismuth/ECNF and carbon nanotubes/ECNF composites were studied towards both $\mathrm{VO}^{2+} / \mathrm{VO}_{2}{ }^{+}$and $\mathrm{V}^{2+} / \mathrm{V}^{3+}$ redox couples. Based on the finding that superior performance on $\mathrm{V}^{2+} / \mathrm{V}^{3+}$ was achieved by bismuth/ECNF and $\mathrm{VO}^{2+} / \mathrm{VO}_{2}{ }^{+}$by carbon nanotubes/ECNF, they developed an asymmetric battery sandwiched with the selected catalyst layers on the respective sides of electrodes. Fig. 4(f) presents improved EE at various current densities by this asymmetric battery ( $82 \% \mathrm{EE}$ than $79.7 \% \mathrm{EE}$ for pristine $\mathrm{CF}$ at $\left.100 \mathrm{~mA} \cdot \mathrm{cm}^{-2}\right)$. After this initial battery testing that packed the ECNF/catalyst layer between commercial felt and membrane, Jing et al. [124] found an excessive hydrophobicity of electrospun fibres due to their higher graphitisation degree, which led to low utilization of the large geometric surface area given by the smaller fibre diameter; demonstrating that though it is possible to make high surface area electrodes via electrospinning, the surface functionality has to be taken into account to allow for good wetting and therefore maximisation of the effective electrochemical surface area. In this respect, the addition of cerium (IV) oxide $\left(\mathrm{CeO}_{2}\right)$ to electrospun fibres was discovered to boost its electrochemical performance activity, which was ascribed to the enhancement of wettability and subsequent recovery of the electrochemical active surface area [124].
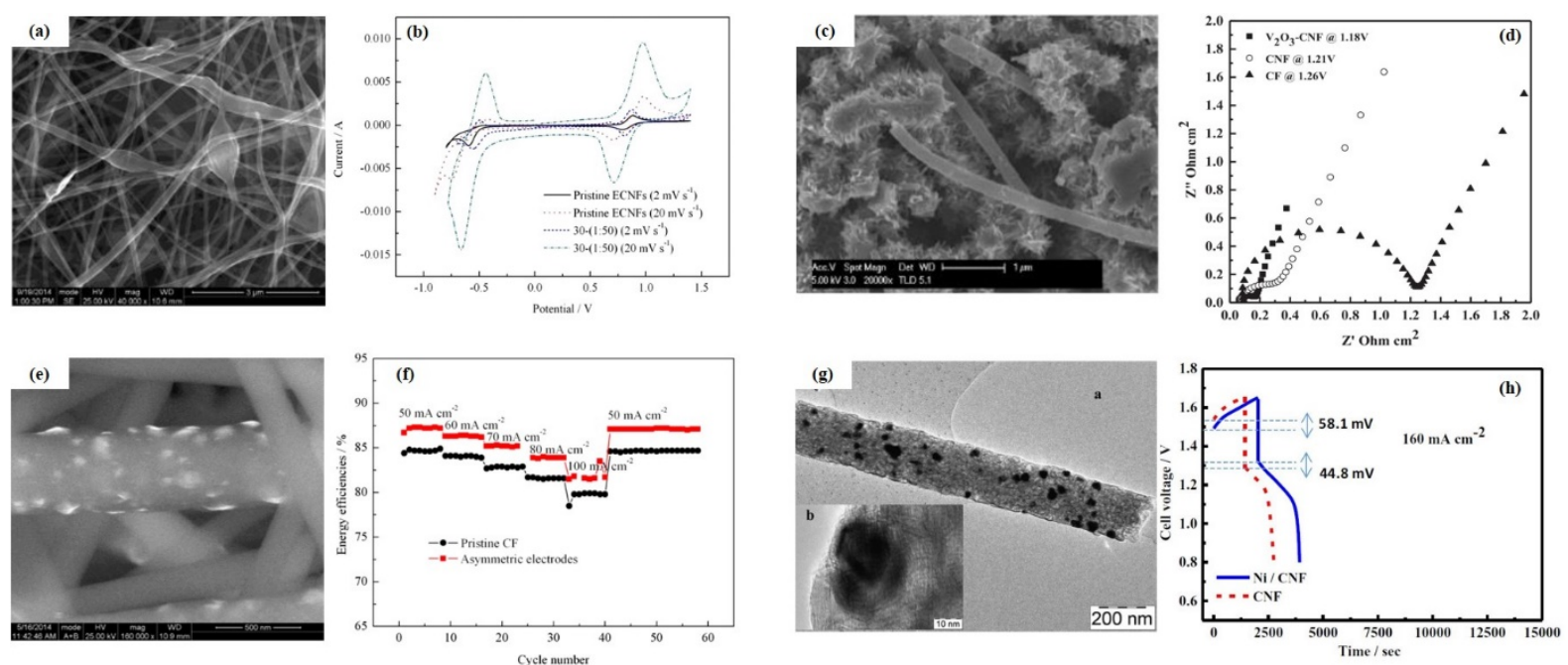

Fig. 4. (a) SEM image and (b) CV comparison of ECNF with graphite nanopowders [128]. (a) shows some examples of the 'beading' that can sometimes occur in electrospinning, as outlined in Section 2. (c) SEM image and (d) EIS comparison of urchin-like nanofibres [125]. (e) Z-contrast image and (f) EE comparison in various current density of bismuth embedded ECNF [120]. (g) LRTEM and HRTEM (inset) images and (h) discharge curves of nickel/ECNF composite [127]. 
In general, more effort has been given to catalysing the negative electrode couples in VRFBs, as the slower electron transfer rate of $\mathrm{V}^{2+} / \mathrm{V}^{3+}$ makes it the limiting electrode during battery cycling. The low reversible potential of this redox couple $(-0.225 \mathrm{~V}$ vs. SHE) relative to the hydrogen evolution reaction also adds a degree of difficulty in adding catalysts to the negative electrode, as the catalyst choice must not enhance the HER (and ideally suppress it) in order to minimise Coulombic inefficiencies and degradation of the aqueous electrolyte. Various catalysts, including rutile titanium oxide $\left(\mathrm{TiO}_{2}\right)$ [121], zirconium dioxides $\left(\mathrm{ZrO}_{2}\right)$ [122] and RGO [123] were mixed into the spinning solution and formed into an ECNF/catalyst layer to accelerate the reaction rate. Aside from lowering the energy barrier, graphene oxide dramatically increases the active surface area by raising the Brunauer-Emmett-Teller (BET) surface area of ECNF from $57.8 \mathrm{~m}^{2} \cdot \mathrm{g}^{-1}$ to $177.4 \mathrm{~m}^{2} \cdot \mathrm{g}^{-1}$ with a subsequent enlarged double-layer current evident in the CVs, attributed to its buckled structure and the improved hydrophilicity from the oxygen content of its surface functional groups [123]. Nitrogen functional groups have also been reported as an effective modification to enhance ECNF electrocatalytic performance [136]. The nitrogen doping was achieved through the addition of urea into the polymer dispersion solution and again in the subsequent carbonisation process. The nitrogen atoms created more defect edges and promoted wettability of electrospun nanofibres, and hence improved its performance towards electron and mass transfer. A common method of improving the performance of carbon electrodes for VRFBs is to heat-treat them in air to add oxygen functionality; though this increases the wettable surface area, it has recently been suggested by Singh et al. [137] that nitrogen doping of carbons can have a catalytic effect for the V redox couples, beyond that of simply increasing the surface area with oxygen treatment. They found that though their N-doped carbon papers had an electrochemical surface area (ECSA) 16 times less than that of oxygen treated papers, there was superior catalytic activity towards the $\mathrm{VO}^{2+} / \mathrm{VO}_{2}{ }^{+}$couple for the former, meaning N-doping of materials for RFBs electrodes should be further pursued in order to advance the state-of-the-art from the simple air/heat treatments most widely applied in the literature (for example, as outlined in an early work from Sun and Skyllas-Kazacos [138]).

As well as acting as an active additive in conjunction with the commercially available carbon felt or paper, electrospinning can also be used to create free-standing electrodes to entirely replace the traditional materials in an RFB cell. Mechanically flexible ECNF with $\mathrm{Mn}_{3} \mathrm{O}_{4}$ catalyst was shown to function as a free-standing electrode by Di Blasi et al. in 2017 [126]. The $\mathrm{Mn}_{3} \mathrm{O}_{4}$ nanoparticles formed during carbonisation further boost the electrocatalytic activity 
of electrospun material for both vanadium couples. The coupling of $\mathrm{Mn}_{3} \mathrm{O}_{4}$ with ECNFs accomplished higher wettability due to its oxygen content, improved charge transfer due to higher carbon graphitisation in the fibres, as well as more active catalytic sites from the spinel structure of manganese (II, III) oxide, hence increased the EE from $70 \%$ for the commercial carbon electrode, to $80 \% \mathrm{EE}$ for ECNF and $81 \% \mathrm{EE}$ for $\mathrm{Mn}_{3} \mathrm{O}_{4}$ modified $\mathrm{ECNF}$ at $80 \mathrm{~mA} \cdot \mathrm{cm}^{-}$ ${ }^{2}$ discharge current density. Even though $\mathrm{Mn}_{3} \mathrm{O}_{4}$ as a catalyst for VRFB was reported as early as 2012 by Kim et al. [139], it was an interesting finding in this electrospinning paper [126] that the improvement on the adoption of ECNF was much more significant than adding $\mathrm{Mn}_{3} \mathrm{O}_{4}$, which only marginally improves the performance further. The same year, Busacca et al. [125] infused vanadium salts into the polymer spinning solution and formed urchin-like nanofibres with rhombohedral $\mathrm{V}_{2} \mathrm{O}_{3}$, as shown in Fig. 4(c). This novel structure of ECNF- $\mathrm{V}_{2} \mathrm{O}_{3}$ composite showed advanced performance with a lower charge transfer resistance of $0.12 \Omega \cdot \mathrm{cm}^{-2}$ at $1.18 \mathrm{~V}$, compared with $0.2 \Omega \cdot \mathrm{cm}^{-2}$ and $1.16 \Omega \cdot \mathrm{cm}^{-2}$ for pristine ECNF and commercial CF (Fig. $4 \mathrm{~d}$ ), respectively. This advanced performance was attributed to not only the catalysing effect of $\mathrm{V}_{2} \mathrm{O}_{3}$, but also an altered more graphitic structure of the supporting fibres, which showed improved conductivity of $0.034 \mathrm{~S} \cdot \mathrm{cm}^{-1}$ to $0.065 \mathrm{~S} \cdot \mathrm{cm}^{-1}$ when combined with the $\mathrm{V}_{2} \mathrm{O}_{3}$. Another highlight of this work was the substitution of polymer PAN for thermoplastic polyvinylpyrrolidone (PVP) to avoid the use of hazardous DMF solvents. More detailed discussion on carbon precursors was given in the section 3.5, though a comparison with PAN after carbonisation was not made, and so it is difficult to know if there was an effect from different surface functionality of the carbonised PVP (though XPS showed a similar C:O ratio to other papers using PAN). A supplementary function was also discovered with nickel [127], which multiplied the polarity of electrospun carbon and ensured a larger electrode-electrolyte double-layer interface. The increased wettability by the nickel-generated oxygen functional groups assisted electrolyte accessibility to the electrode surface as well as the catalysing sites. These features gave Ni/ECNF a high effective surface area, delivering electrochemical functionality that enhanced redox activity and electron conductivity. The transmission electron microscopy (TEM) characterization shown in Fig. $4(\mathrm{~g})$ proved that the nickel particles were embedded and created pores/defects in the carbon nanofibres, which created catalytic sites and increased surface area. This high active surface area Ni/ECNF promoted both cathode and anode reactions, where the charge/discharge overpotential was reduced by $102.9 \mathrm{mV}$ compared to pristine ECNF (Fig. 4h), and obtained an impressive EE of $68 \%$ at $160 \mathrm{~mA} \cdot \mathrm{cm}^{-2}$. 
In summary, the catalyst additives in electrospinning are valued for their electrocatalytic activity as well as a side function of better utilization or extension of ECNF surface area by their improved wettability or pores-forming ability. Another advantage is the enhanced stability due to a uniformly embedded structure of nanoparticles in the ECNF, in contrast to other synthesis methods (e.g. separate electrodeposition and hydrothermal reaction). Unfortunately, the catalyst performance is difficult to compare between work without actual flow battery tests, as the testing protocols, such as the scan rates in CVs and the polarization voltages in EISs, vary from study to study. A standard testing protocol for catalyst studies in RFBs is imperative to communicating and exchanging advancements in the field. The intrinsic mechanism of metal catalysing the redox reactions has not been the main focus in the papers of metal and ECNF composites, as the metals with other synthesis method were proved effective previously. However, the kinetics/mechanisms of improving battery performance of the metal catalysts in a dense and nano-scaled ECNF are possibly different from a more porous and micro-scaled structure of CF. More generally, full flow battery testing of ECNFs is vital as the field advances as it is only then that the mass transport properties of the electrodes can be evaluated in addition to their catalytic activity in static electrolyte systems. This way, the varied microstructure and chemical compositions of ECNF when electrospinning with additive salts might prove beneficial or detrimental to its overall performance in a full cell. With consideration of all aspects of the properties of these materials in future work, we believe the potential of ECNF/catalysts composites could be realised in working RFB systems.

\subsection{Microstructure and image-based modelling}

As RFBs are flow-through operation devices, the microstructural properties of the electrodes, such as the pore size, porosity, tortuosity, and connectivity play an important role in porous material behaviour. SEM is a widely applied imaging technique to evaluate the microstructural properties of a wide variety of materials. However, as it is a 2D imaging method, it is inherently limited to evaluation of the top or bottom surface of electrodes, or a cross-section through a selected point to give through-plane morphology, and as such, internal heterogeneities can be missed. This is particularly key for the electrospinning technique as it is a dynamic procedure and is sensitive to the developing spin conditions through the process as more material is deposited (in the through-plane direction), and any heterogeneities can be further exaggerated by the following carbonisation step. Additionally, macroscopic measurements, such as total porosity, are non-representative when assessing only one face of the electrode. Though sliceand-view focused ion beam (FIB) SEM can be used to resolve the whole structure, it is a time 
consuming and destructive technique. To solve these problems, non-destructive insight by Xray $\mathrm{CT}$ has recently been used to assess the microstructure of the commercial electrode materials for RFBs [132,140-142], and more recently for electrospun materials (requiring nano-CT due to the smaller fibre size [132]). X-ray CT has also been used for non-aqueous RFBs to screen the commercially available electrodes based on the simulated electrolyte flow pattern and other fluid transport properties [134]. Though this technique has obvious advantages in imaging and visualising the 3D structure of porous media, the true power of the technique comes from it providing a virtual volume on which image-based modelling can be carried out. The flow, pressure and velocity distributions can be numerically simulated based on the actual structure and a computational fluid dynamics model. Finally, image-based models can also have the capability to predict performance and to correlate the battery performance with its microstructure and synthesis parameters [134].

For the transport properties and electrolyte flow patterns, the LBM, Darcy's law and Fick's law are most utilized for modelling RFBs. Kok et al. [111] addressed the structural heterogeneity of electrospun porous mats through LBM modelling. They found a significantly altered surface morphology during the carbonisation, but with a preserved inner structure, leading to large through-plane heterogeneities. Before carbonisation, the fibre diameter displayed little variation in-plane (fibre layers) or through-plane (thickness) and hence the surface value of fibre diameter could represent the bulk property. After carbonisation, there was a significant deviation comparing both the bottom and top surfaces of electrospun samples with fibre sizes within the mat, and a reduction of fibre size towards the edges from the middle was encountered. Fibre size would be underestimated around $25 \%$ by surface characterization alone, demonstrating the benefit of the 3D X-ray CT approach. They also observed a significant variation in local pore size distribution through the plane of the electrode, which has consequences for the flow characteristics of the electrolyte in this media. LBM simulations revealed a coupling of permeability to local porosity and that the heterogeneities in the material could lead to severe channelling of the electrolyte through only a small fraction of the electrode. The difference in apparent porosity of the microstructure from a simulated mercury intrusion porosimetry (MIP) experiment (Fig. 5c) and that determined by a local thickness method (Fig. $5 \mathrm{~d}$ ), shows the effect of shielding and bottlenecking of larger pores by smaller pores: that is, the true and inherent porosity measured by a local thickness algorithm (Fig. 5d) might not be fully accessible to the electrolyte due to smaller pores upstream of the flow. This can have drastic consequences for the performance of the RFB, resulting in regions of reactant starvation 
adjacent to the areas of high-velocity flow, as well as underutilisation of the full electrode volume. The shielding and bottlenecking phenomenon could be aggravated by inconsistencies in the synthesis of electrospun materials and the anisotropies present in commercial (woven) CF materials, demonstrating the need for accurate control and rational design of microstructure in order to produce efficient electrodes for RFBs. This is particularly important when operating at higher current densities, where good mass transport and electrode utilisation become critical, and additionally in low states of charge (SoC) where the relative concentration of the reactants in the available electrolyte volume is diminished significantly. Large mass transport overpotentials caused by these two operating conditions (and unoptimised microstructures) requires long constant voltage steps to be added to the cycling protocol to access the full charge of the electrolyte inventory, or a reduced SoC operating window must be used for shorter charge/discharge cycles. As the electrolyte is often a significant proportion of the cost of RFB systems (particularly for VRFBs), tailoring the microstructure of the electrodes to minimise mass transport overpotential, and therefore maximise the practical operating SoC window, effectively means that the electrolyte utilisation is higher (or the battery is charged and discharged more quickly for the same utilisation), and therefore electrode microstructural properties can have a direct effect on the cost of RFB systems. 

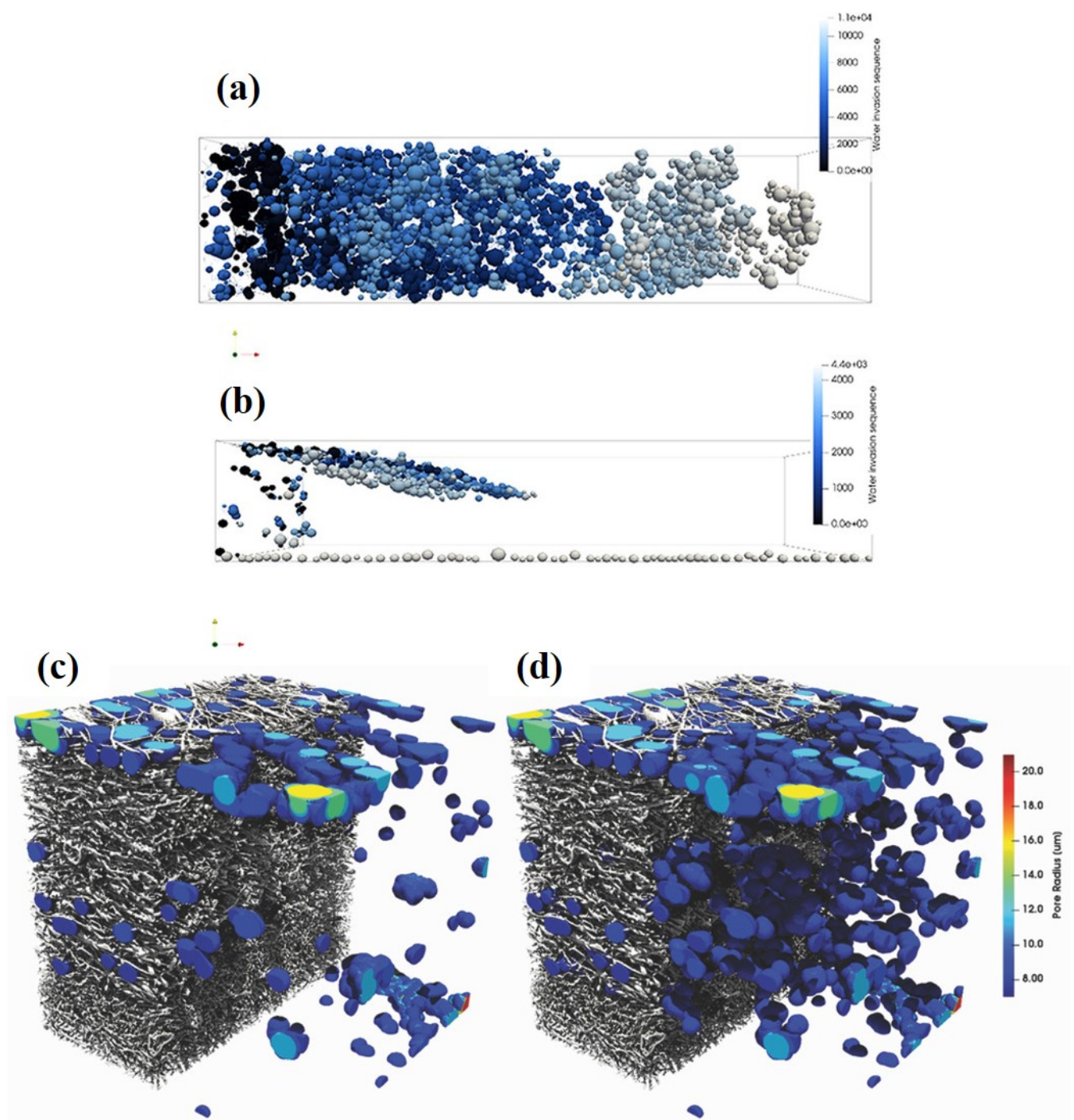

Fig. 5. Modelled invasion pattern of the electrolyte positions in an electrode at breakthrough condition of (a) CF and (b) ECNF-CF. Reproduced from Ref. [143] with permission from John Wiley and Sons. Local pore sized determined by (c) MIP and (d) local thickness method. Reproduced from Ref. [111] with permission from John Wiley and Sons.

The channelling phenomenon through modelling was also demonstrated experimentally by Fetyan et al. [143]. A pore network model was developed based on the CF electrodes [131,144], and then was used by Fetyan et al. [143] for the comparison of electrolyte transport through the $\mathrm{CF}$ and their designed electrospun material. This pore network model could extract properties such as electrolyte invasion behaviour and saturation information with a reduced computational demand compared with direct numerical simulation on each voxel of a CT volume. Their low-cost electrospun fibres were synthesised by substituting the PAN carbon precursor with carbon black powder, and obtained a high surface area electrode with a rough and twisted surface. The fibres showed improvement on the performance of the CF in the low current density regime; ECNF on CF achieved $75 \% \mathrm{EE}$ at $20 \mathrm{~mA} \cdot \mathrm{cm}^{-2}$ compared to $70 \% \mathrm{EE}$ of CF. Unfortunately, this low-cost carbon-carbon composite as a free-standing electrode was not capable of operating at a discharge current density higher than $100 \mathrm{~mA} \cdot \mathrm{cm}^{-2}$ because of the 
sharp performance degradation below $50 \% \mathrm{EE}$ at $80 \mathrm{~mA} \cdot \mathrm{cm}^{-2}$. X-ray CT imaging and modelling shed light on this poor battery performance; the pore size distribution for the CF was between 25 - $75 \mu \mathrm{m}$ but was below $20 \mu \mathrm{m}$ for their electrospun electrode, where a smaller pore size leads to large invasion pressure and entrance capillary pressure [143]. Most RFBs have no back-pressure system, which results in a much lower saturation of the electrospun electrode. Fig. 5( $\mathrm{a}$ and $\mathrm{b})$ show the break-through point, which is the point of electrolyte reaching the opposite face of the electrode. An extremely low saturation of electrospun electrode $(3.8 \%$ compared to $31.1 \%$ for $\mathrm{CF}$ ) was observed, as there formed a continuous pathway through the electrode at the point of electrolyte invasion, leading to the vast majority of the electrolyte being channelled through a small fraction of the pore structure, which was also found in the work of Kok et al. [145] discussed previously.

\subsection{Structure and morphology design}

Electrospinning allows tailored bulk solutions of organic polymers to be formed into thin fibres, but also has the versatility to alter fibre diameter, pore size, and pore shapes through adjusting parameters during the fabrication process. As discussed in the previous section, the low saturation and heterogeneity of the flow pattern is closely related to the microstructural properties, and is detrimental to the performance of the battery. Altered morphologies were observed in some electrospun materials with additives; for instance, the addition of carbon black resulted in a rough and twisted surface [143] and vanadium salts produced urchin-like fibres [125]. Nevertheless, the microstructural properties were not the main focus of the work and were not taken into account when analysing the battery performance. The concern of the low permeability of dense electrospun material was first addressed in 2018 [111,143], supported by image-based modelling. In the following publications, many parameters were considered to achieve an ideal structure: the electrospun solution variations included polymer concentration [130], multi-polymer precursors [146,147], novel electrospun operation setup and parameters include horizontally opposite-electrospinning [147] and the use of high-speed rotating drum collectors [132].

Though electrospinning allows for control of the microstructure, the dynamic nature of the technique means that careful optimisation of the spinning and carbonisation parameters is required (and sometimes needs to be altered throughout the spinning process) to avoid any undesired variation in structural inconsistency, particularly as thick fibre mats are spun and accumulation of insulating material changes the electric field properties. This can result in 
heterogeneity as described by Kok et al. [111], which could cause the issues of channelling and shielding observed experimentally in Fetyan's work [143]. With careful control of the spinning process, it is possible to avoid the issues and synthesise electrospun electrodes with consistent fibre diameters and a small pore size distribution [132]. A consistent microstructure was successfully synthesised by using more refined operation with a feedback control process to keep constant operation conditions as more insulating material was deposited through the process. Furthermore, on top of the consistency, alignment in the in-plane direction was achieved by using a rapidly rotating drum collector to increase the permeability. Fig. 6 demonstrates how the controlled electrospun fibres (EE-C) narrow the fibre diameter distribution compared to the heterogeneous electrospun fibres (EE-H), and additionally avoided anomalous large fibres such as those seen in EE-H [132]. Additionally, it was found that the alignment delivered a sufficient anisotropy and obtained the highest permeability in the direction parallel to the aligned direction of the fibres, which added a new understanding to the effect of alignment to that of the elongated graphite planes and improved conductivity in the aligned direction demonstrated by Flox et al. [115]. Alignment was also applied by Zhang et al. [148] to address the low permeability issue in the direction of electrolyte flow and the aligned ECNF resulted in an increased limiting current density, 25\% higher than that of carbon paper.
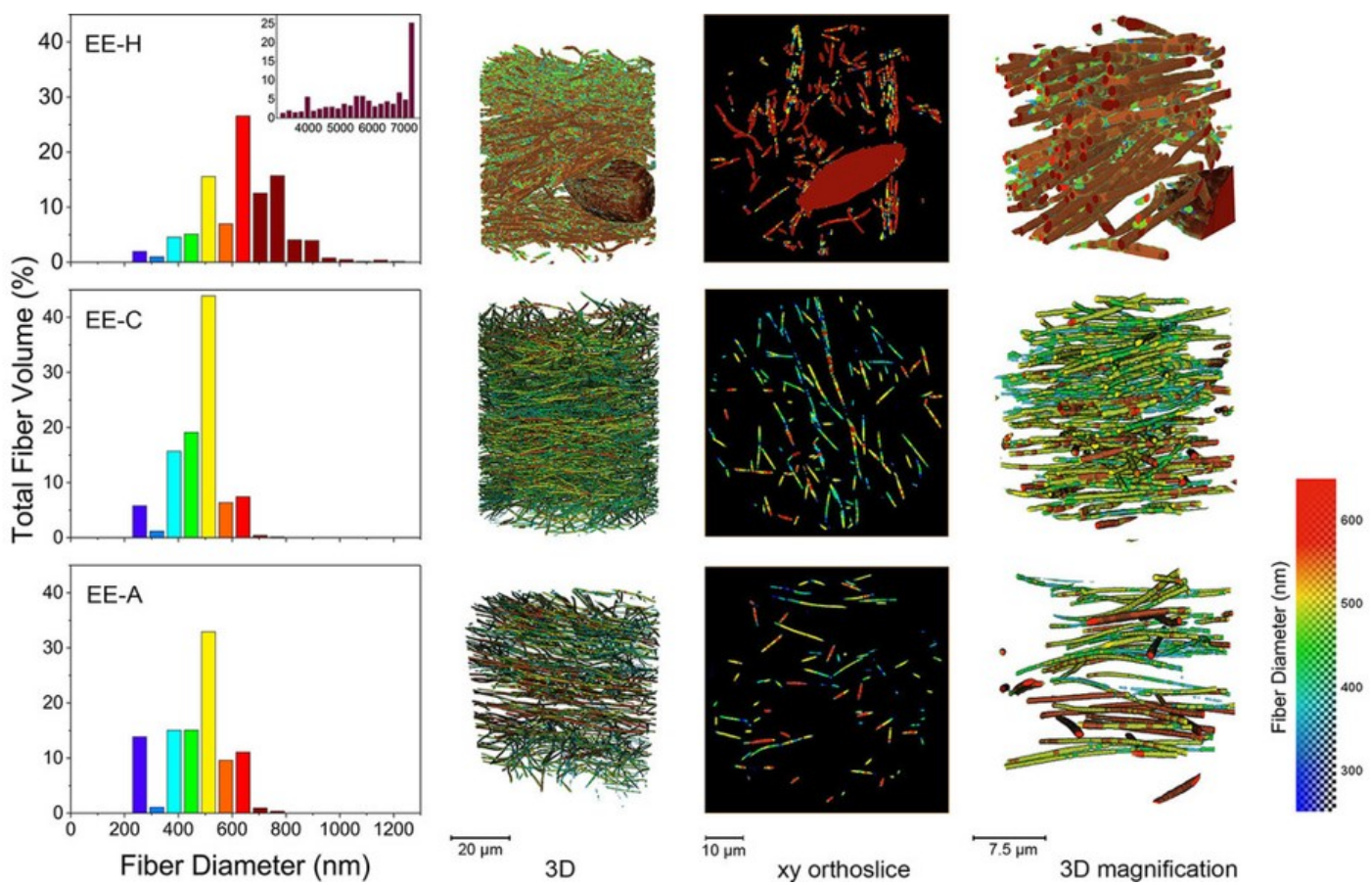

Fig. 6. Fibre diameter distributions of electrospun material without control (EE-H), with control (EE-C) and aligned (EE-A) calculated by the local thickness method (left), and the 3D rendering of the whole volume (left) and magnified area (right), as well as one example xy orthoslice (middle) for each sample. Reproduced from Ref [132] with permission from John Wiley and Sons. 
For the widely applied ECNF/CF composite, the improved battery performance has also been ascribed to the variation of microstructure. $\mathrm{Wu}$ et al. [135] described it as a dual-layered electrode (Fig. 7a) with ECNF as a catalyst layer and commercial felt electrode with larger pores as a backing layer. They explained this dual-layer structure could match the architecture of a fuel cell, where the gas diffusion layer (GDL) used for uniform reactant distribution corresponded to the backing phase and the reactions mainly occurred in the ECNF phase, analogous to the catalyst layer in a fuel cell. Therefore, the high catalytic activity of ECNF was maintained, and the low saturation issues could be resolved by increasing the inlet electrolyte homogeneity through the better distribution properties of the backing layer. When performing at a high current density, the poor battery performance of the commercial carbon electrode and ECNF was attributed to the activation loss and concentration loss, respectively. The combination of both by this dual-layer structure provided a synergistic performance enhancement effect and offset both disadvantages. Through screening of the backing layer, flow rate and flow field geometry, the best battery performance with optimized selections reached high $\mathrm{EE}$ of $80.2 \%$ at $240 \mathrm{~mA} \cdot \mathrm{cm}^{-2}$ current density.
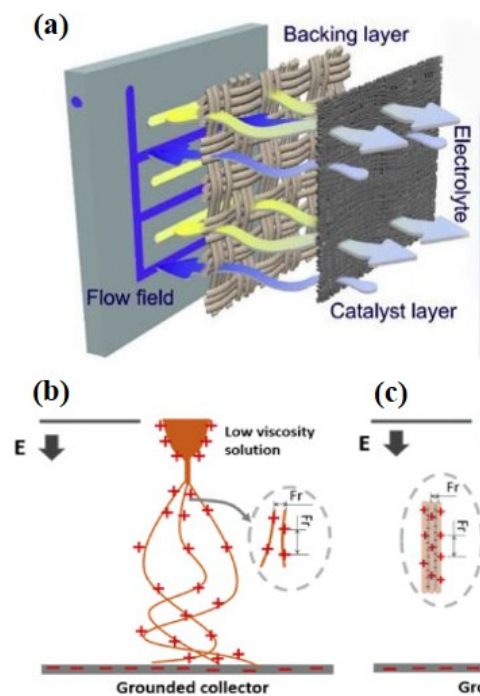

(c)

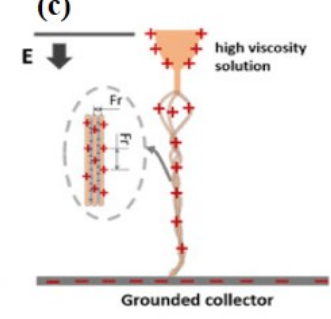

(f)

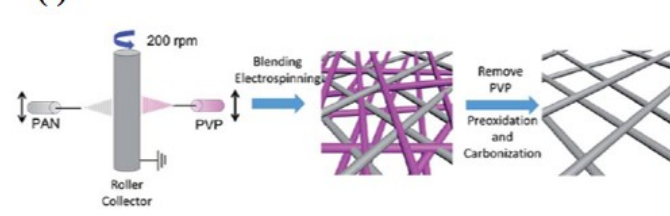

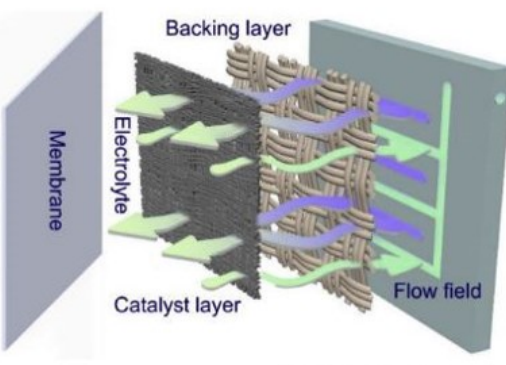

(d)

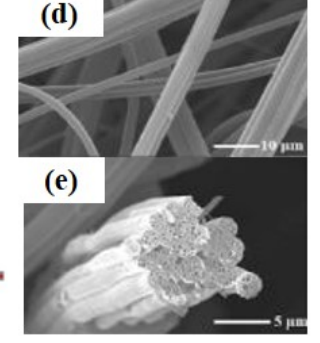

(g)

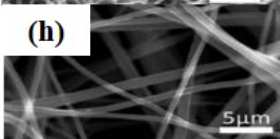

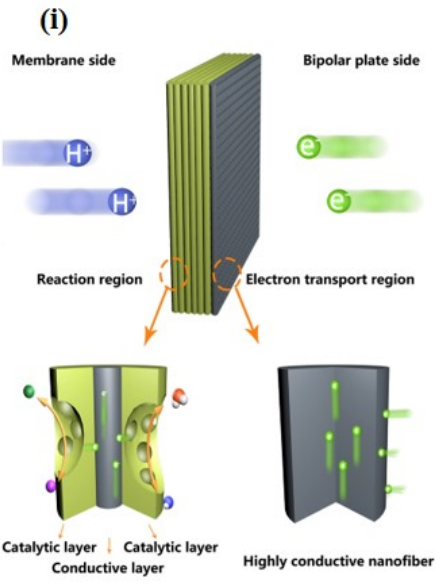

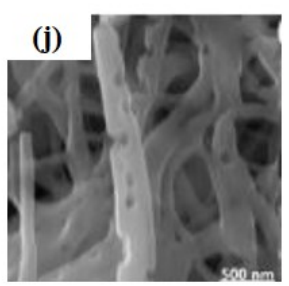

Fig. 7. (a) The design of dual-layer structured RFB [135]. The formation mechanism of (b) electrospun fibres, and (c) electrospun fibre bundles, (d) LRSEM image and (e) HRSEM image of bundled electrospun fibres [146]. (f) Synthesis procedures, HRSEM image of (g) normal ECNF and (h) bi-electrospun carbon nanofibres. Reproduced from Ref. [147] with permission from The Royal Society of Chemistry. (i) The schematic on the micron-scale and the nano-scale, and (j) HRSEM image of the integrated electrode [149]. 
However, the configuration of a commercial CF as a backing layer and an ECNF catalyst layer reduces the inherent benefit of using ECNF, in that free-standing electrospun fibre mat provides a thin and high surface area electrode which can greatly reduce the overall stack size in RFBs. To maintain the benefits of a thin ECNF electrode but with the improved mass transport of electrodes with larger voids (as in commercial CF electrodes), Xu et al. [149] recently used a novel approach where the structure of the electrode was altered from the region closest to the current collector (defined as the 'electron transfer region' by the authors) to the region closest to the separator (defined as the 'reaction region'). They used a high concentration of PAN (14 $\mathrm{wt} \%$ ) to provide larger void space, but during the $\mathrm{ES}$ process added a $\mathrm{CaCO}_{3}$ salt towards the end of the spinning, which introduced nanoporosity to the fibres in the region closest to the separator (after carbonisation), resulting in a higher surface area in the 'reaction region'. With this concept and morphology shown in Fig. 7( $\mathrm{i}$ and $\mathrm{j})$, the reaction region had a micron-scale void structure and a nano-scale porous structure of $150 \mathrm{~nm}$ pores evenly distributed on the fibre surface, and the electron transfer region was a thin film with the typical ECNF solid fibres and next to the bipolar plate. Through changing the electrospinning solute from PAN to the mixture of PAN and $\mathrm{CaCO}_{3}$ salt during the multi-step electrospinning procedure, a continuous phase transformed from the region closest to the current collector, minimising the contact resistance, into a higher surface area layer in the region closest to the separator. In the 'reaction region', $\mathrm{CaCO}_{3}$ decomposed during the carbonisation step, resulting in $\mathrm{CO}_{2}$ etching, which formed additional nano-pores in the catalytic layer as well as oxygen-rich functionality surrounding the nano-pores. Along with the abundant active sites provided by the enhanced surface area and wettability of the 'reaction region', the thin dense film in the 'electron transfer region' facilitated the contact area between the electrode and current collector. This indiscrete electrospun material with distinct morphology change through the thickness of the electrode pushed the battery performance with ECNF electrode to the best-reported value of $80.2 \% \mathrm{EE}$ at $250 \mathrm{~mA} \cdot \mathrm{cm}^{-2}$ and peak power density of $702.98 \mathrm{~mW} \cdot \mathrm{cm}^{-2}$. The concept and rationale of this electrode design were tested by comparing the electrode performance with different variables of this modification, and they showed inferior battery performance of the control group with a single synthesis step/variable (including no electron transfer region, elimination of the reoxidization step and low carbonization temperature), proving the advantageous properties of this novel design [149].

Efforts to control the structure and design morphology are an effective solution in improving battery performance. A more straightforward approach was taken by Xu et al. [147] to increase 
the distance between fibres to reduce the pressure drop. They successfully expanded the void space by oppositely bi-electrospinning PAN and a sacrificial polymer PVP with processing steps shown in Fig. 7(f), and achieved larger voids (Fig. 7h) than would normally be obtained with electrospinning (Fig. 7g) after its removal. The CV comparison of the bi-electrospun and normal ECNF electrodes showed the bi-electrospun electrode underwent higher activation loss due to its lower surface area and fewer functional groups. However, their material performed better in the battery single cell test, with twice the utilization of electrolyte capacity and $77.9 \%$ EE with $11.1 \%$ improvement at $60 \mathrm{~mA} \cdot \mathrm{cm}^{-2}$, thanks to its higher permeability and improved mass transport. The expansion of voids approach was also found in a porous bundle structure (Fig. 7d), which was synthesized by electrospinning a bi-component solution of PAN and polystyrene (PS) and resulted in a four times larger void distance [146]. Unlike the basic sacrificial phase in the previous study, PS can not only form large fibre bundles and control bundle sizes, but also facilitated the formation of internal channels in the fibres, as shown in Fig. 7(e), which augmented the specific surface area. The evolution of self-assembly bundles was dominated by the polymer solution viscosity because the attraction between two sticky jets was higher and led to intertwining during the spinning process. Fig. 7(c and b) illustrate the formation of separated fibres with a low viscosity precursor and bundles with high viscosity. PS could be able to be spun at eight times the viscosity of pure PAN but still form stable bundles, compensating the unsteady electrospun performance of high viscous PAN solution. The electrolyte utilization capacity at $100 \mathrm{~mA} \cdot \mathrm{cm}^{-2}$ current density was doubled with this PS/PAN bi-blending electrode, which benefited from the lower concentration polarization resistance and enhanced mass transport performance, especially at higher current densities. Both papers discussed above increased the porosity and hence the permeability; while the fibres generated by the second polymer jet of PVP in bi-electrospun ECNF were removed to produce larger pores, inevitably reducing the volumetric surface area (surface area/volume) [147]. On the other hand, the bundled structure formed because of increased viscosity by mixing PS with PAN has larger 'fibre size', which results in larger voids and less surface area [146]. Though the surface area reduction was offset by the additional area in the internal channels, consistency of the bundles was varied, as shown in Fig. 7(d), with different numbers of fibres forming bundles with diverse diameters [146]. This heterogeneous microstructure with bundles could lead to channelling and shielding within the electrode.

To date, the progress of RFB electrodes by rational design of ECNF microstructure has shown significant promise. The excellent electrolyte utilization with ECNF electrodes has pushed the 
operating battery current density to a higher range $\left(>200 \mathrm{~mA} \cdot \mathrm{cm}^{-2}\right)$. The concept of the duallayered structure explained the improved performance by sandwiching ECNF between commercial electrodes and membrane [135]; however, the application of electrospun material should not be restricted to either in a sandwich form or as a free-standing electrode. Novel designs benefiting from the flexibility of electrospinning have shown some recent promise for effective electrodes made purely from ECNFs. Nonetheless, there are still experimental challenges in achieving a theoretical optimised structure. The dynamic electrospun process strongly relates to the variables in the system; therefore, there is increasing complexity and difficulty in producing consistent results when varying many parameters including additives, multi-polymers, multi-jets and post-treatments. Feedback control systems could be considered for lab-scale experiments and could prove especially useful for industrial-scale production in the future. Furthermore, a combination of optimised morphology for mass transfer, catalysis for kinetics and modelling for performance prediction could boost the battery performance to a new level soon.

\subsection{Conclusions and perspectives}

Aside from the research angles reviewed herein, the sustainability of the petrochemical-derived PAN polymers often employed in RFB electrodes has driven recent research interests towards biomass-derived precursors, which have been pursued in many other energy-relating fields [150-152]. Lignin, originating from plants and collected as a waste by-product, has been explored in RFB recently. Vivo-Vilches et al. [153] screened and tested three types of lignin: Kraft (KL), ethanol organosolv (EOL) and phosphoric acid lignin (PL) as the precursors and added polyethylene oxide as plasticizer for the ECNF formation. They found a positive correlation between the molecular weight of lignin and fibre mechanical stability. The fibres derived from EOL, which has the lowest molecular weight of the three in this study, experienced severe pyrolysis and were unable to resist thermal treatment. Unfortunately, they did not measure the jet viscosity, as this is suspected to have an impact due to the lower viscosity of the polymer with smaller molecular weight. However, this phenomenon still requires further research to understand, especially with the presence of plasticizer. In this work, the electrospinning applied voltage was also optimized and the necessity of pre-treatment in air was evaluated. The carbon nanofibres made from PL and KL electrospun under $9 \mathrm{kV}$ with air stabilization pre-treatment had impressive CV electrochemical performance for vanadium redox couples, suggesting lignin is malleable for electrospinning and has potential as an RFB electrode [153]. Lignin also stands out for its unique chemistry, which produces abundant 
oxygen functional groups delivering a hydrophilic surface with rich active sites. Further treatment to introduce $\mathrm{N}$ and $\mathrm{P}$ to provide more polar surface and active sites for vanadium couples was studied by Ribadeneyra et al. [154] through thermal decomposition of $\mathrm{NH}_{4} \mathrm{PF}_{6}$. With comparison to a commercial carbon fibre fabric sheet, the KL-derived ECNF was found to have a higher surface area and activity. However, on P-doping the performance of the ECNF was worse than the commercial CF (with the former having a higher level of P-doping, due to the larger number of oxygen functional groups in the starting material), attributed to reduced conductivity due to doping induced blockage of micro/mesopores and electron transport. Their work was the first to evaluate biomass-derived ECNF for VRFB electrode in a single cell battery test, and presented a comparable battery performance of lignin-derived electrode with commercial electrode material for capacity utilization, energy efficiency and stability. Although long-term testing is required, their results indicated the potential of biomass-derived electrodes for RFBs. However, the selection and intensity of surface functionalization should be considered to avoid undesirable side effects, as well as synthesis conditions to maintain a mechanically and chemically stable microstructure in future studies.

To date, applications of ECNF for RFBs have indicated these novel materials can lead to an improvement on commercially available electrode materials and have promise for application in commercial RFB systems. The foremost studies were driven by a theoretical principle and explored the capability of electrospinning as a production method for electrode materials for RFBs. However, electrochemical testing is often limited to CV and EIS in static electrolyte for comparisons with commercially available electrodes, and full cell testing is required to fully evaluate the mass transport performance of ECNF materials. ECNFs were first utilized as a catalyst layer for their high surface-area, and subsequent modifications with other catalysts or atom doping advanced the performance. The battery performances of the catalysts/ECNF composites are presented in Fig. 8 (blue), which all display steady EE of around $75 \%$ below $100 \mathrm{~mA} \cdot \mathrm{cm}^{-2}$, and a moderate performance fade with increasing current density. In order to maximize the power density of stacks, ECNFs as a free-standing electrode have been proposed. The first free-standing ECNF only accomplished $74.1 \% \mathrm{EE}$ at $40 \mathrm{~mA} \cdot \mathrm{cm}^{-2}$, demonstrating the importance of microstructure in issues of shielding, channelling and bottlenecking of flow, highlighted by image-based modelling. Subsequently, researchers have started to attach importance to the alteration of morphology and microstructure, with novel designs enhancing the performance of pristine ECNFs, comparable to that achievement by the use of ECNFs as a thin catalyst layer on commercial CF electrodes (Fig. 8 green). Notably, several recent structure 
designs (Fig. 8 pink) have been successful in pushing the operating current density to 250 $\mathrm{mA} \cdot \mathrm{cm}^{-2}$ while preserving an outstanding energy utilization with over $80 \%$ EE. With regards to performance degradation, all carbon-based electrodes for aqueous flow batteries, both electrospun and carbon felt, are resistant to cycling within a reasonable voltage window. Comparing with the benchmark performance of a 6 MWh VRFB cycled for more than 200,000 cycles in 2005 [155], electrospun material in RFBs is still in its early stages and needs further study. Nevertheless, its superior catalytic activity towards reaction redox couples is a potential solution to the high overpotential of commercial electrodes under high discharge current density. The best performing ECNF electrodes in future studies are predicted to be those that optimise the microstructure in combination with a catalytic enhancement to ensure efficient operation over the activation and mass transport regions of the operating RFB.

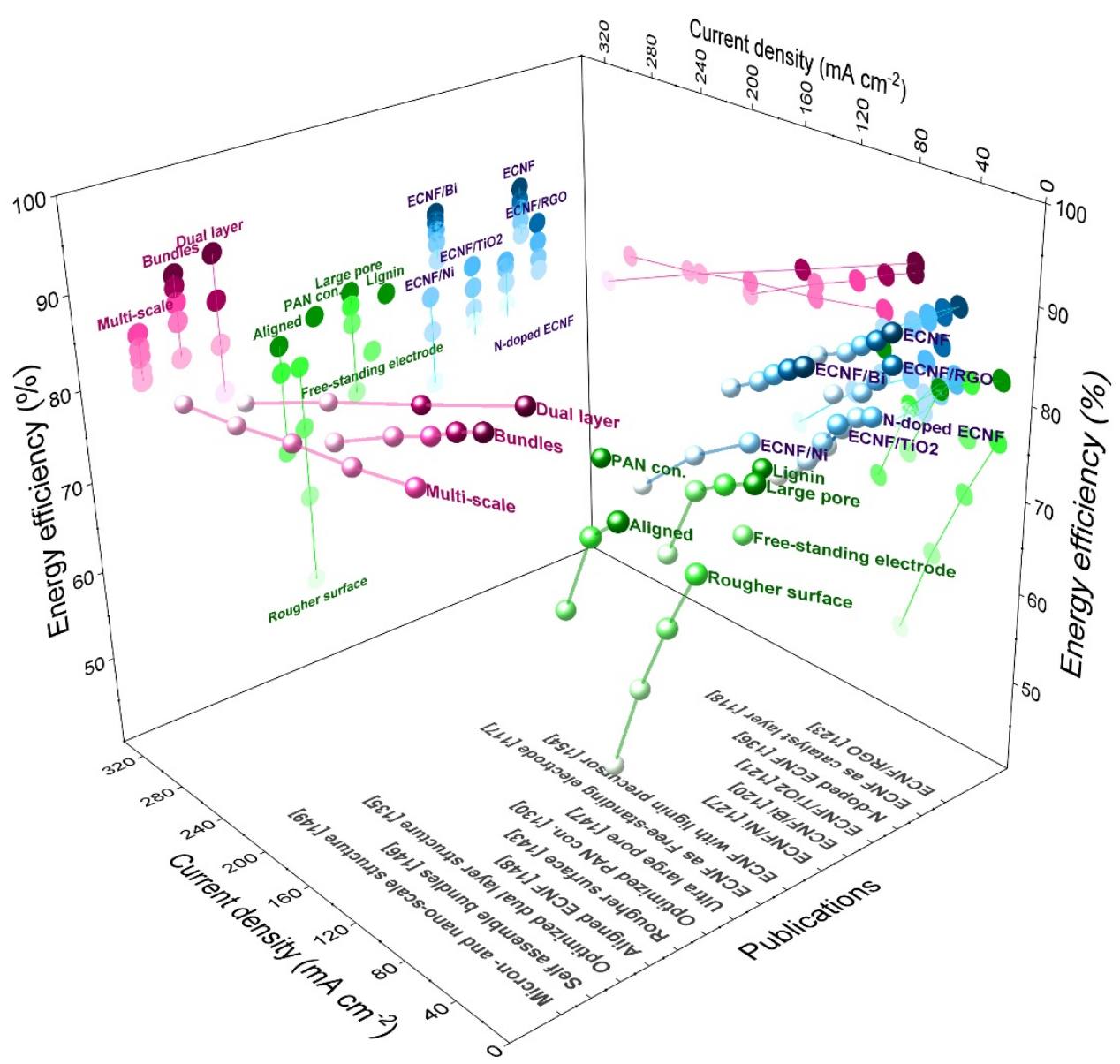

Fig. 8. A conclusion of battery performance (energy efficiency vs. current density) in most publications discussed in this section (some work is not presented for a clearer view); Blue: ECNF or ECNF/catalysts composites as a catalyst layer; Green: ECNF with modified structures as a free-standing electrode; Pink: Free-standing electrode of ECNF with novel structures with notable performance. '2D projections' of energy efficiency vs publication number and energy efficiency vs current density are shown on the back wall of the figure for ease in extracting values from the data. 


\section{Low-temperature fuel cells applications}

\subsection{Introduction}

Another energy conversion technology where electrospinning finds application is in the processing of electrodes for low-temperature fuel cells, including proton exchange membrane fuel cells (PEMFC) and alkaline fuel cells (AFC). Fuel cells employ gas diffusion layers (GDLs) within the structure of the electrode to enable even and efficient delivery of reactant gases (often hydrogen and oxygen) to the catalyst surface, as well as aiding in the removal of product water from the system, and so the microstructure of the GDL directly influences the performance of fuel cells. Additionally, the conductive pathway of the current produced travels through the GDL to the current collectors, and therefore the GDL material must have good electrical conductivity. For this reason, the GDL is often composed of carbon fibre papers or cloths. For hydrogen fuel cells, the oxygen reduction reaction (ORR) taking place at the cathode constitutes the limiting factor for both efficiency and cost, due to sluggish ORR kinetics and the high price of platinum-based cathode catalysts, respectively. To reduce the amount of Pt used in fuel cells, the catalysts tend to be in the form of well-dispersed nanoparticles on a conductive and porous support such as carbon black.

Besides the outstanding efficiency of Pt in catalysing the ORR, which leads to a 4-electron pathway and high current densities, its stability under normal operating cathode conditions tends to decay after some time due to Pt agglomeration, Pt dissolution and carbon support corrosion, all of which lead to loss of active surface area and therefore electrocatalytic activity towards ORR. As well as Pt active sites modification, $\mathrm{CO}$ poisoning can reduce the activity of the catalyst [156]. These phenomena translate into reduced performance over time and possible replacement in aged commercial FCs, potentially increasing the economic cost to the final user after time [156-161]. Hence, over the years, different approaches have been developed to improve Pt stability under ORR conditions, or even replace it with lower cost and efficient oxygen electrocatalysts (noble metal-free materials) [162-164].

In recent years, the exploration and development of potential substitutes of $\mathrm{Pt}$ and their supports has focused on the design of new nanostructured materials, with the aim of achieving the required properties for efficient electrocatalysis: high electric conductivity, porosity, surface area and concentration of active sites for the ORR [162-164]. To that extent, electrospinning has stood out as a low-cost and scalable manufacturing process to produce nanofibre electrodes for ORR electrocatalysis [156-164]. Electrospinning is a highly versatile technique that 
enables the design and optimisation of electrocatalytic nanomaterials by varying the processing parameters [156-164], as outlined in Section 2.

Researchers have used electrospinning for manufacturing fuel cell electrodes using three main approaches: (i) to produce tailored microstructures for the GDL architecture; (ii) to engineer cathode catalysts which can provide higher stability to Pt; and (iii) to process self-supported transition metal and/or heteroatom doped carbon nanofibres (CNF). The achievements of the main studies and discoveries of these approaches will be outlined and discussed in the next subsections of this review, and the performance of the various materials discussed is summarised graphically within this section, and tabulated in the supporting information.

\subsection{Electrospinning for GDLs}

The basic role of the gas diffusion layer, as its name suggests, is to provide gas access via diffusion to catalyst sites under the flow field ribs (land areas). Without such a porous spacer, a proportion of the catalyst would be inactive due to gas starvation. However, being placed between the gas flow channels and the catalyst layer means the GDL must support the transport of all species involved in the reaction (except protons). This means that GDLs must be electronically and thermally conductive; highly porous to provide gas pathways; mechanically robust to provide support to the membrane-electrode assembly over the channel region; and, perhaps the biggest challenge, they must be able to manage any liquid water within the cell resulting from the combination of water produced by the ORR and high humidity required by the ionomeric membrane.

Conventional GDLs universally consist of 7-10 $\mu \mathrm{m}$ diameter carbon fibres. This is because they are made from commercially available pre-carbonized fibres, which are made by carbonizing a continuous strand of extruded polyacrylonitrile, yielding a spool of high quality, graphitic fibre. GDLs are made by chopping the carbonized PAN and assembling into a fibrous mat. The fibres are then bound together using various approaches, such as hydroentanglement or polymeric binders. A thorough overview of traditional GDL manufacturing is given by Mathias et al. [165].

Over the last several years, researchers have begun to investigate whether smaller fibres might be better suited for GDLs, and electrospinning is the ideal platform from which to launch such studies. For a given porosity, smaller fibres mean smaller pores, and the curved nature of the fibre surfaces impact the capillary behaviour via the Purcell effect [166], so smaller fibres also exhibit increased hydrophobicity [167]. Since one of the main roles of GDLs is water 
management, it follows that GDLs with smaller hydrophobic fibres would be less prone to water flooding, resulting in lower overall water saturations. In traditional GDLs, PTFE is often added to increase hydrophobicity, at the expense of sacrificing a proportion of the porosity.

Todd and Merida [168] were the first to investigate the potential of electrospun GDLs (eGDLs) in a PEM fuel cell. They observed lower performance, resulting from both increased mass transfer and ohmic resistance, but did optimistically note that operation at higher current density was more stable. They proposed that the eGDLs retained less water which hindered performance by reducing the ionic conductivity of the membrane. The increased mass transfer resistance was not explained, and the structural and transport properties of their eGDLs were not provided. In a follow-up paper [169], they explored the impact of anisotropic fibre orientation on performance and found that highly aligned fibres, oriented perpendicular to the flow field, out-performed commercial materials, though no justification was given for this enhancement. More recent work by Bazylak and co-workers, discussed below, highlights the importance of electrical conductivity of the layers. It is therefore plausible that the improvement of their highly-aligned materials resulted from the more dense packing of the fibres and subsequent improvement in fibre-to-fibre contact. This is supported by the substantially better performance in the ohmic region.

Bazylak and co-workers have undertaken a detailed study of eGDLs, including parametric studies of GDLs properties [170] and radiography of in-situ water distributions [171]. They found that large fibre-to-fibre spacing led to poor electrical conductivity, which influenced the performance negatively. Conversely, they found that the eGDLs had worse mass transfer performance than conventional GDLs, which they attributed to the accumulation of liquid water in the catalyst layer due to the excessive hydrophobicity of the eGDL layers. They used a surface functionalization technique to render the fibres hydrophobic, which was perhaps too effective. The fact that GDLs must mediate so many varied transport processes presents a challenge since all aspects must be balanced. Salahuddin et al. [172] recently proposed eGDL materials with superhydrophobic surfaces, but they did not test cell performance, so going to extremes in any given direction may not be the correct approach. Another intriguing finding coming from the above studies is that highly-aligned fibres, oriented perpendicularly to the flow-field, provide stronger mechanical support to the membrane electrode assembly (MEA) when compared to alignment of the fibres with the flow fields where uneven compression from the land/channel contact could cause cleaving of the electrode along the direction of alignment, 
as indicated by the radiographs. This could also explain the improved cell performance in the ohmic region.

One well-known downside of using large fibres for GDLs is that physical contact between the GDLs and the MEA is poor. To remedy this, a microporous layer (MPL) is often added to the MEA-facing side of the GDL. The MPL has a similar structure to the catalyst layer (CL), but no catalyst, and is very flat, so interfaces well with the CL. The MPL also intrudes deeply into the GDL, thereby bridging the electrical and thermal connection between the CL and GDL. In the above-mentioned studies, eGDLs were used with no MPL since the smaller fibres create a smoother surface for contacting the catalyst layer. One of the early applications of electrospun layers in PEMs was to replace the MPL with an electrospun equivalent (eMPL) while retaining the commercial GDL. Duan et al. [173] leveraged the smaller pores and higher hydrophobicity and reported improved peak performance compared to a standard microporous layer. Li et al. [174] explored the reason behind this improvement in more detail and identified the increase in diffusion through the eMPL, which has higher porosity and pores that are large enough to avoid Knudsen diffusion resistance. Lee et al. [175] extended basic construction of the eMPL by adding some partial hydrophilic functionality, which retains water near the membrane and is believed to provide a humidification buffer to maintain ionomer hydration, or to wick excessive water from the catalyst layer (probably both). This approach is similar to adding hydrophilic powders to a traditional MPL [176]. Finally, Balakrishnan et al. [177] created an eGDL with a gradient in fibre size, and therefore pore size, which essentially mimics a GDLMPL dual-layer structure. It showed very similar performance to a commercial dual-layer material and vastly outperformed a commercial GDL with no MPL.

In summary, the smaller fibres and resultant pore sizes of the electrospun GDLs and MPLs are generally beneficial to fuel cell performance. In the GDL, the increased hydrophobicity prevents water from flooding the layer and maintains good oxygen mass transfer. In the MPL, the smaller fibres create a better interface with the catalyst layer, while the pores are large enough to avoid Knudsen effects. Since there are so many competing objectives in the GDLMPL role, a balance must be struck, thus aiming for extremes in any given area, such as hydrophobicity or high porosity, can have negative unintended consequences. There is certainly much room for improvement in this area as a relatively limited number of reports are available in the literature. 


\subsection{Electrospun nanofibres as support for Pt and Pt alloys}

To increase stability and decrease mass loading of Pt for ORR applications, research has been devoted to investigating the support surface effect on the stability of Pt, correlated with controlling structure/geometry and electronic state of Pt particles [156]. Tailoring of desired support properties leads to stabilization of the surface energy interaction between fibres and supported $\mathrm{Pt}$, as well as exposure and accessibility for oxygen to active sites, and an enhancement of the triple-phase point (absorbed oxygen, catalytic sites, electrolyte) during ORR catalysis $[156,157,179,158-164,178]$. Similar studies have also been developed with Pt alloys with other noble metals $[178,179]$. In this sense, the use of electrospinning can lead to interesting fibre morphologies, including hollow and multi-layered, that can potentially enhance Pt stability and improve oxygen accessibility to catalytic sites [156-164].

In 2015, Cavaliere et al. [157] used PAN electrospun nanofibres as a template to produce Sbdoped- $\mathrm{SnO}_{2}$ hollow nanofibres, via calcination of the polymeric phase, obtaining diameters of less than $100 \mathrm{~nm}$ and high porosity. The $\mathrm{Sb}$ doped- $-\mathrm{SnO}_{2}$ fibres were then decorated with $\mathrm{Pt}$ via chemical reduction of $\mathrm{H}_{2} \mathrm{PtCl}_{6} \cdot 6 \mathrm{H}_{2} \mathrm{O}$, leading to controlled geometry of Pt nanoparticles, easy accessibility of oxygen and good ORR performance under acidic conditions [157]. (Supplementary Information, Table S.1). However, stability (40\% ECSA loss after 10,000 cycles) and initial ECSA were found to be lower than commercial Pt catalyst. Fuel cell testing led to a maximum power density of $0.61 \mathrm{~W} \cdot \mathrm{cm}^{-2}$ and open circuit potential of $0.96 \mathrm{~V}[156,157]$.

A similar approach using Nb-doped $\mathrm{SnO}_{2}$ as a support for Pt was reported by Savych et al. [158], obtaining similar results to Cavaliere et al. [157] (Table S.1). A few years later, similar work used $\mathrm{Nb}$-doped- rutile $\mathrm{TiO}_{2}$ electrospun nanofibres as the support for Pt nanoparticles [180]. The doping effect of $\mathrm{Nb}$ on rutile $\mathrm{TiO}_{2}$ increased the electrical conductivity of the electrospun dioxide fibres and changed the surface energy, achieving homogeneous distribution and stability of Pt nanoparticles [180]. With this approach, reduction of Pt mass loading $\left(60 \mu \mathrm{gPt}_{\mathrm{P}} \cdot \mathrm{cm}^{-2}\right)$ was achieved as well as relatively high retention of mass activity after 6,000 cycles (64\%), in a half-cell setup under acidic conditions [180]. In Fig. 9, micrographs and electrocatalytic performance of this electrospun material are shown as a good example of these type of materials used as a support for Pt. 

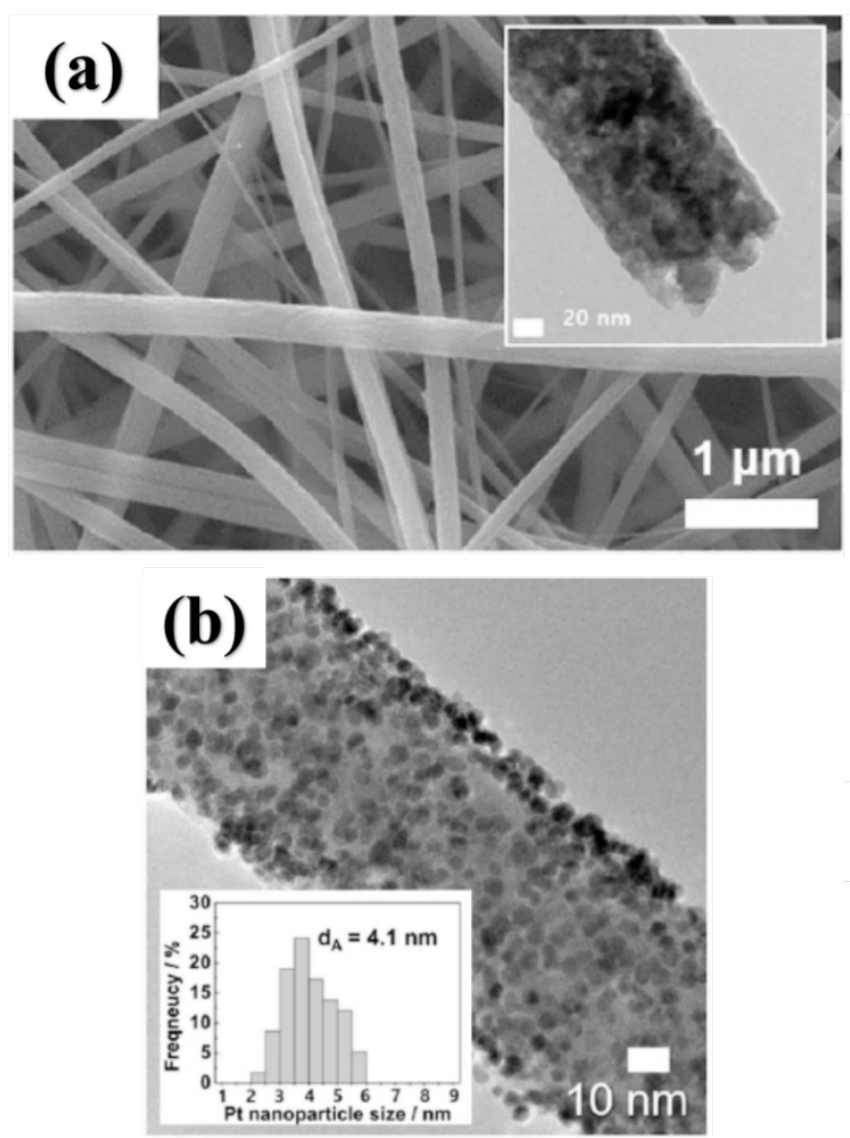

(c)

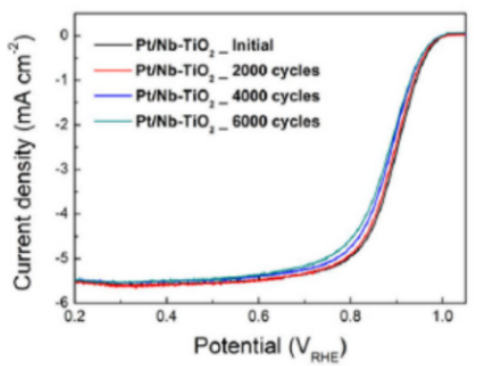

(d)

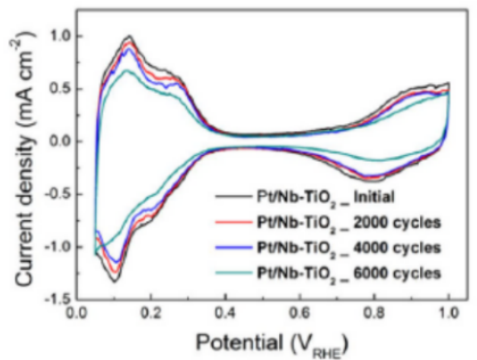

(e)

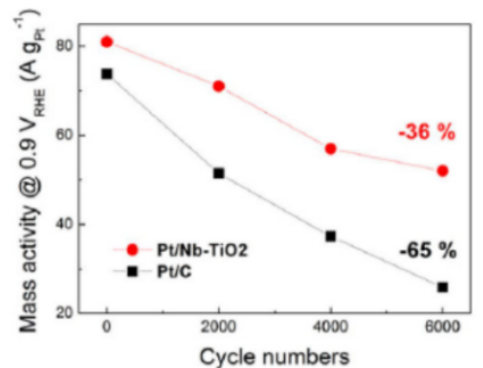

Fig. 9. (a) SEM and TEM (inset) micrographs of $\mathrm{Nb}-\mathrm{TiO}_{2}$ nanofibres, (b) TEM micrographs of Pt supported on $\mathrm{Nb}^{-\mathrm{TiO}}{ }_{2}$ nanofibres catalyst (inset: Pt nanoparticle size distribution), (c) LSV stability test, (d) CV stability before, during and after ADT and (e) mass activity @ 0.9 V vs. RHE before, during and after ADT. Reproduced from Ref. [180] with permission from Nature Publishing Group.

Electrospun $\mathrm{TiO}_{2}$ nanofibres/wires were also studied by Deng et al. [178] as a support for a PtAu alloy active material for the ORR. After calcination, a thermal reduction with $\mathrm{H}_{2}$ was performed, diminishing mass loading to $7.03 \%$ loading of Pt and achieving a similar ORR activity and enhanced stability compared with commercial $20 \% \mathrm{Pt} / \mathrm{C}$ electrocatalyst standard (Table S.1). This improvement of mass loading and enhanced ORR performance was attributed to an enhancement of conductivity of the oxide fibres due to the presence of Au. The material's superior stability was ascribed to the PtAu alloy considering a synergistic effect due to inert nature of $\mathrm{Au}$ and $\mathrm{Pt}$ that enhances the resistance in acidic conditions due to its electron configuration, providing $\mathrm{Pt}$ with these desirable properties for ORR electrocatalysis. Regardless of enhanced properties, to make more commercially feasible fuel cells, it is desirable to not only decrease Pt mass loading but also avoid using other expensive elements that cannot assist in addressing economical practicality of this technology [178].

Other popular electrospun materials used as a support for Pt are nitrides [181,182]. Titanium nitride nanofibres were synthesized via a facile three-step process: electrospinning, thermal 
stabilization and nitridation, by Kim et al. [181] Properties of this material were optimized to achieve an ECSA of $181.44 \mathrm{~m}^{2} \cdot \mathrm{g}^{-1}$ and a Pt content of $20 \%$. Electrochemical testing encountered very similar performance compared to a commercial catalyst ( $E_{\text {onset }} \sim 0.98 \mathrm{~V}$ vs. RHE, $E_{1 / 2} \sim 0.88 \mathrm{~V}$ vs. RHE, $J_{\mathrm{D}} \sim 5.5 \mathrm{~mA} \cdot \mathrm{cm}^{-2}$ ) and better stability (retained current density after 6,000 cycles $\sim 91 \%$ ) compared to similar measurements for $\mathrm{Pt} / \mathrm{C}$ standard reported elsewhere [164]. Exposed catalytic sites due to porosity of this material combined with surface energy provided to Pt from the support could be the main factors that helped this material to retain current density after accelerated degradation tests (ADT) [181].

Another example of electrospun nitrides are the vanadium nitride (VN) nanofibres used as a support for Pt by Kim et al. [182]. Though good electrocatalytic performance with lower Pt mass loading was achieved ( $15 \mathrm{wt} \% \mathrm{Pt})$, current density retention was determined to be around $60 \%$ after $60 \mathrm{~min}$ of chronoamperometric testing. Even though their stability testing outcome was better compared to $\mathrm{Pt} / \mathrm{C}$, it is very doubtful that this material could be able to retain electrocatalytic properties towards ORR on long-time operation (Table S.1).

Niobium carbide $(\mathrm{NbC})$ nanofibres were synthesized as a support for platinum due to their intrinsic chemical stability [160]. Niobium carbide fibre support provided Pt nanoparticles with better electrochemical properties due to the synergetic catalysis and structure stability for ORR. However, mass loading had to be increased (30 wt $\% \mathrm{Pt})$ to achieve comparable performance in full-cell and half-cell testing. Low surface area correlated with the nonporous nature of carbides leads to higher Pt mass loading for this material, perhaps contributing to the relatively low (31\%) ECSA retention after ADT 10000 cycles, probably due to increased Pt nanoparticle agglomeration and lower contact between the $\mathrm{Pt}$ and $\mathrm{NbC}$ surface, resulting in a higher concentration of Pt nanoparticles not supported on the surface of carbide [160].

However, though oxide and nitride supports are demonstrated in the literature, the most researched support for Pt is carbon [156]. In the case of the electrospun carbon supports, there are many examples in the literature [162,183-185]. A simple electrospinning, graphitisation, and deposition process was reported by Wang et al. [162], supporting Pt on N-doped- C porous nanofibres. The performance of this supported Pt in acidic media was comparable with benchmark Pt/C black catalyst and after ADT the half-wave potential shifted by only $21 \mathrm{mV}$ after 1,000 cycles [162]. Retention of the fast kinetics of the reaction was attributed to the influence of the N-doped-C nanofibres on the stability of Pt [162]. Electrocatalytic activity $\left(E_{\text {onset }} 0.949 \mathrm{~V}\right.$ vs. RHE, $E_{1 / 2} 0.773 \mathrm{~V}$ vs. RHE, $\left.n>3.9\right)$ and stability $\left(\Delta E_{1 / 2}=21 \mathrm{mV}\right.$ after 1,000 
cycles) of Pt on mesoporous $\mathrm{C}$ nanofibres has been shown to be correlated with surface area dependant geometry of deposited platinum nanoparticles [156], [162]. Even though stability was achieved, Pt mass loading remained equal to the $\mathrm{Pt} / \mathrm{C}$ standard ( $20 \mathrm{wt} \% \mathrm{Pt})$.

Hierarchical Pt grafted on $\mathrm{N}$-doped mesoporous $\mathrm{C}$ nanofibres were reported with a high surface area $\left(685 \mathrm{~m}^{2} \cdot \mathrm{g}^{-1}\right)$, low Pt content $(2.04 \% \mathrm{Pt})$, and enhanced electrochemical performance in a PEMFC ( $E_{\text {onset }} 0.993 \mathrm{~V}$ vs. RHE, $E_{1 / 2} 0.911 \mathrm{~V}$ vs. RHE, max. power density $0.427 \mathrm{~W} \cdot \mathrm{cm}^{-2}$ ) [184]. These properties were achieved by surface functionalization of the nanofibres before deposition of $\mathrm{Pt}$ nanoparticles via a wet chemistry reduction process. This obtained hierarchical Pt grafted on N-doped electrospun fibres with an enhanced triple-phase boundary for ORR, leading to a more efficient absorption-reaction-desorption rate [184].

Hollow porous carbon nanofibres have also been synthesized using a coaxial electrospinning process, using two electrospinning solutions with different polymers (PAN and PVP) [183]. After a stabilization process, PVP was decomposed leaving hollow PAN nanofibres for subsequent carbonisation and deposition of Pt nanoparticles (mass loading $19.78 \mathrm{wt} \% \mathrm{Pt}$ ) [183]. The electrochemical performance in a half-cell setup was lower $\left(E_{\text {onset }} 0.895 \mathrm{~V}\right.$ vs. RHE, $E_{1 / 2}$ $0.763 \mathrm{~V}$ vs. RHE, ECSA $\left.73.96 \mathrm{~m}^{2} \cdot \mathrm{g}^{-1}\right)$ compared to Pt/C ( $E_{\text {onset }} \sim 1.0 \mathrm{~V}$ vs. RHE, $E_{1 / 2} \sim 0.8 \mathrm{~V}$ vs. RHE, ECSA $\left.\sim 80 \mathrm{~m}^{2} \cdot \mathrm{g}^{-1}\right)$ [156] but better stability was achieved $\left(\Delta E_{1 / 2}\right.$ shifted $57 \mathrm{mV}$ vs. $165 \mathrm{mV}$ after 10,000 cycles).

Another mesoporous hollow C nanofibre used as a support for Pt nanoparticles was reported by Karuppanan et al. [185] This composite exhibited high surface area $\left(780 \mathrm{~m}^{2} \cdot \mathrm{g}^{-1}\right)$, low platinum content $(2.53 \%)$ and synergetic electrocatalytic activity due to $\mathrm{N}$-doping of $\mathrm{C}$ fibres (2.8\% pyridinic). In addition, similar onset and half-wave potentials ( $E_{\text {onset }} 0.99 \mathrm{~V}$ vs. RHE, $E_{1 / 2} 0.92 \mathrm{~V}$ vs. RHE) were measured compared to a $\mathrm{Pt} / \mathrm{C}$ standard. Though this material had a low Pt content, a higher ECSA $\left(98.8 \mathrm{~m}^{2} \cdot \mathrm{g}^{-1}\right)$ was demonstrated. Accelerated degradation tests showed enhanced retained current density ( $86.1 \%$ after 30,000 s) and low half-wave potential shifting (28 mV after 10,000 cycles), proving enhanced stability of platinum in this matrix due to stabilization of nanoparticles and high accessibility for $\mathrm{O}_{2}$ on the $\mathrm{C}$ support.

Palladium contained in $\mathrm{C}$ electrospun nanofibres were synthesized as a support for $\mathrm{Pt}$, accomplished via electrospinning and pulsed electrodeposition of the active Pt particles [186]. As a Pt-group element, palladium has the same outer-shell electron configuration as Pt and has been proven to be capable of acting as an efficient electrocatalyst for ORR. With this simple synthesis process, mass loading of platinum was reduced and cell activity remained stable, $\mathrm{Pd}$ 
could have also acted as an active site for oxygen, but unfortunately, Pd loading was not disclosed by the authors $[156,186]$. Electrochemical properties measured in a PEMFC lead to comparable properties (ECSA $53.3 \mathrm{~m}^{2} \cdot \mathrm{g}^{-1}$, OCP $0.98 \mathrm{~V}$, maximum power density $0.682 \mathrm{~W} \cdot \mathrm{cm}^{-}$ ${ }^{2}$ ) with the state-of-the-art electrocatalyst and higher stability with low mass loading. Stability assessed via ADT shows a retained power density of $87 \%$ after 10,000 cycles; this could be attributable to multiple catalytic sites $(\mathrm{Pt}$ and $\mathrm{Pd}$ ) and chemical stability provided by $\mathrm{Pd} / \mathrm{C}$ surrounding Pt nanoparticles.

Via a two-step synthesis method by electrospinning and atomic layer deposition, successful deposition of Pd nanoparticles on N-doped C nanofibres was reported by Khalily et al. [187]. Atomic layer deposition (ALD) gives the unique advantage of precisely controlling the growth of nanoparticles on the surface of any substrate. The porosity of nanofibres can be diminished by atomic deposition, as exposed in this work (surface area $63 \mathrm{~m}^{2} \cdot \mathrm{g}^{-1}$ ), but with the advantage of lowering Pd loading $(0.324 \% \mathrm{Pd})$ and providing electrocatalytic activity towards ORR ( $E_{\text {onset }} 0.936 \mathrm{~V}$ vs. RHE, $E_{1 / 2} 0.769 \mathrm{~V}$ vs. RHE) [187]. Similarly to Pt, N-doping on the fibres provides a synergistic interaction with Pd during ORR catalysis; nonetheless, no stability test was shown, which might indicate low stability of this material.

Recently, PtPd alloy nanoparticles were supported on the surface of N-doped C nanofibres by Li et al. [179], exhibiting comparable onset ( $0.970 \mathrm{~V}$ vs. RHE) and half-wave (0.840 V vs. RHE) potentials to $20 \% \mathrm{Pt} / \mathrm{C}$. Again, no stability in acidic conditions was demonstrated in this work, which could hint a poor resistance under acidic conditions [156,179].

The performance of the materials discussed in this sub-section are summarised in Fig. 10(a) shows the relation of Pt mass content with onset and half-wave potentials, showing some materials with lower Pt mass loading can achieve high activity, demonstrated by the onset and half-wave potentials) [156]. Fig. 10(b) shows the correlation between ECSA, $E_{1 / 2}$ and max. power density. It is easy to distinguish that materials with higher OCP have lower power density and vice-versa. Perhaps in order to optimize and improve performance an equilibrated correlation must be reached as seen on the material with ECSA $\sim 55 \mathrm{~m}^{2} \cdot \mathrm{g}^{-1}, \mathrm{OCP} \sim 0.98 \mathrm{~V}$ and max. power density $\sim 0.7 \mathrm{~W} \cdot \mathrm{cm}^{-2}$. 
(a)

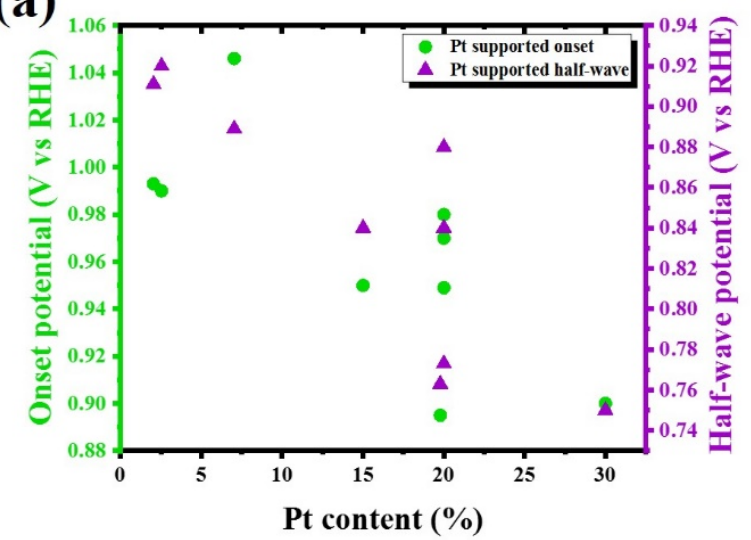

(b)

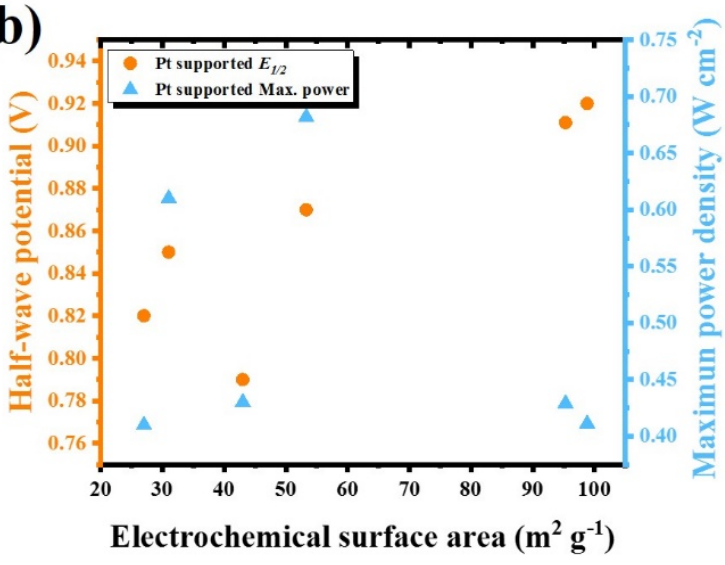

Fig. 10. Electrochemical activity comparison of Pt supported on different materials for cathode application in fuel cells: (a) Pt content vs. onset and half-wave potential and (b) electrochemical surface area vs. half-wave potential and maximum power density in a fuel cell.

\subsection{Self-supported oxygen electrocatalysts}

Platinum-free self-supported ORR electrocatalysts is currently a large and popular field [188]. The focus on developing new and sustainable materials for potential application in fuel cell cathodes improves the economic viability of this technology, making it more attractive for industry and final users $[156,188]$. Transition metal doped carbon materials have been widely studied during recent years, showing promising results for application in devices [188]. Likewise, co-doped C materials with multiple transition metals (iron and cobalt being the most popular elements), and transition metal and heteroatom co-doped $\mathrm{C}$ materials (usually with nitrogen) have received a lot of attention [162-164]. Transition metal-doped C materials regularly exhibit similar ORR electrocatalytic activity to Pt based materials and often better stability, especially when these are C based, doped with Fe and N [189-192]; electrocatalytic activity and stability of these materials is enhanced by Fe-N co-doping of $\mathrm{C}$ fibres. This is mostly a consequence of the presence of $\mathrm{Fe}-\mathrm{N}_{4}$ coordinated sites (similar to haemoglobin) and pyridinic-N sites (electron density redistribution due to difference in electronegativity between $\mathrm{N}$ and $\mathrm{C}$ ) which increases oxygen absorption and can act as catalytic sites, providing low resistance to charge transfer from catalyst to absorbed oxygen [189-192].

Electrospinning is not exempted as a synthesis method for metal and heteroatom-doped C materials, providing the opportunity to manufacture fibres with high surface area and excellent electric conductivity, as well as controlled morphology [189,193,194].

Catalytic site precursors are usually mixed with the electrospinning polymer solution before spinning $[189,193,194]$. Thermal treatment conditions for doping and carbonisation processes play a fundamental role in synthesis. Most commonly, Fe and $\mathrm{N}$ containing $\mathrm{C}$ nanofibres are 
reported with differing treatment steps applied after the electrospinning process (thermal treatment with ammonia, pyrolysis, sintering, impregnation, acidic etching, wet chemistry surface activation, grinding, high energy ball milling, photo-curing, and MOF templating) [189-191]. Regularly, Fe-N, Fe-Co alloy nanoparticles, Fe-Co-N co-doped C nanofibres exhibit similar electrochemical properties to commercial ORR electrocatalysts in acidic and alkaline media and better stability (retained current density $>90 \%$ and $\Delta E_{1 / 2}$ shifting $<10 \mathrm{mV}$ after ADT) [189,194-197].

A very interesting example of this type of materials is shown in Fig. 11, synthesized using electrospinning and precursor coating by Lee et al. [198] The electrospun porous tri-doped fibres clearly show Fe, N and F content on the carbonaceous matrix (Fig. 11(c-f) and linear sweep voltammetry (LSV) measurements show a half-wave potential difference against $\mathrm{Pt} / \mathrm{C}$ of just $11 \mathrm{mV}$ (Fig. 11)). Additionally, with an onset potential of $0.9 \mathrm{~V}$ vs. RHE, diffusionlimited current density of $5.23 \mathrm{~mA} \cdot \mathrm{cm}^{-2}$ and a determined electron transfer number of 4 , these electrospun fibres behave just as well as $\mathrm{Pt} / \mathrm{C}$ according to Fig. 11(e). Finally, stability after ADT (10,000 cycles) shows a shifting of the half-wave potential of only $9 \mathrm{mV}$, much lower than $\mathrm{Pt} / \mathrm{C}$ which $(42 \mathrm{mV})$. In addition to $\mathrm{Fe}-\mathrm{N}_{4}$ coordinated active sites, pyridinic nitrogen and F-doped carbon fibres can act as additional active sites that have shown a synergistic positive behaviour with Fe-N. 


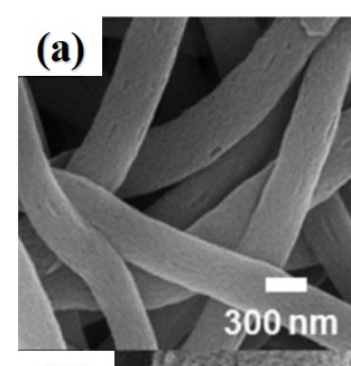

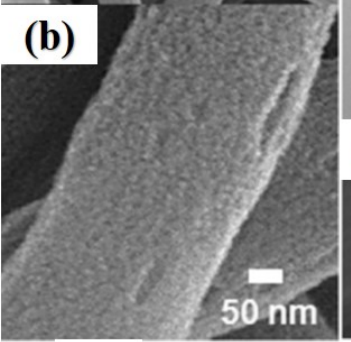

(g)

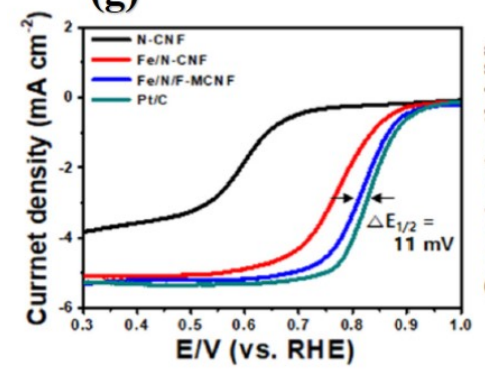

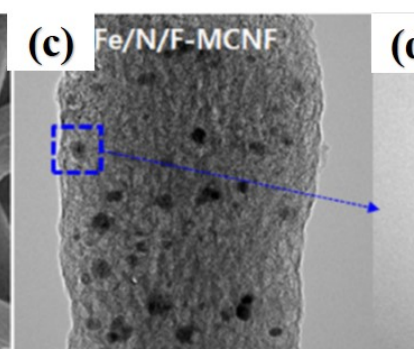

(d)

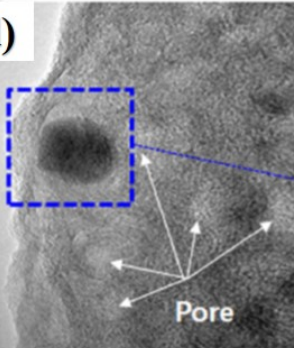

(e)

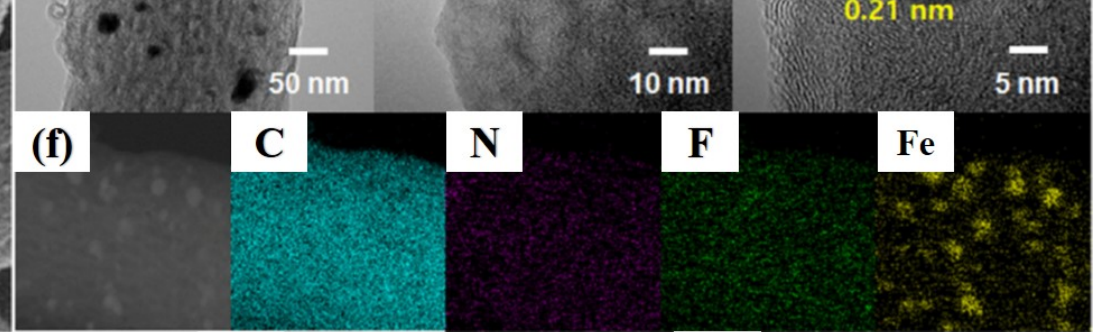

(h)

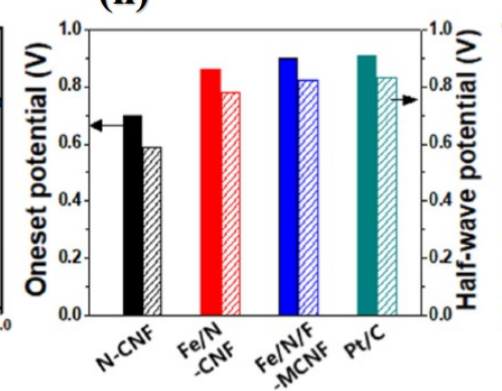

(i)

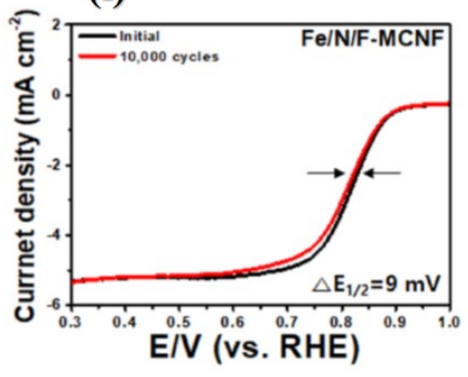

Fig. 11. (a and b) SEM micrographs of tri-doped Fe/N/F nanofibres, (c) to (e) TEM low- and high-resolution micrographs, (f) TEM-EDS mapping, (g) LSV measurement and comparison between similar materials, (h) onset and half-wave potential comparison and (i) stability comparison of the material before and after ADT [198].

Less common electrospun materials have been studied recently such as transition metal oxides [193,199-202]. Advantages of metal oxides are their chemically stable structure, easy tunability of electronic properties and electrocatalytic bi-functionality towards ORR and the oxygen evolution reaction (OER), providing application in water electrolysers (PEMWE) and metal-air batteries [193,199-202]. Huang et al. [34] grew $\mathrm{ZnCo}_{2} \mathrm{O}_{4}$ and $\mathrm{NiCo}_{2} \mathrm{O}_{4}$ nanowires on the surface of electrospun $\mathrm{C}$ fibres with an additional co-precipitation step during synthesis. Efficient oxygen catalysis under alkaline conditions was achieved $\left(E_{\text {onset }}-0.18 \mathrm{~V}\right.$ vs. $\mathrm{Ag} / \mathrm{AgCl}$, $J_{D} \sim 4 \mathrm{~mA} \cdot \mathrm{cm}^{-2}$ and $\left.n_{\text {average }} 3.7\right)$ with low metallic content $(2.1 \% \mathrm{Zn}, 1.1 \% \mathrm{Ni}$ and $2.3 \% \mathrm{Co})$ and better stability than $\mathrm{Pt}$ (retained current density after 10,000 s of $70 \%$ ) [34]. Even though good electrocatalytic activity was demonstrated, stability measured by chrono-amperometric testing after almost $3 \mathrm{~h}$ decreased the diffusion-limited current density by $30 \%$, which on extrapolating to long-term testing could mean the ORR activity would drastically reduce.

Other metal oxides demonstrated in the literature were electrospun manganese oxide nanofibres produced using electrospinning and thermal oxidation, sacrificing the polymeric phase of the nanofibre and allowing the formation of oxides and shrinking of fibre diameter $[193,203]$. 
Nonetheless, as reported in half-cell alkaline testing, electrocatalytic activity towards ORR is not comparable to Pt ( $E_{\text {onset }}-0.25 \mathrm{~V}$ vs. $\mathrm{Ag} / \mathrm{AgCl}$, and $n \sim 3.7$ ) [203] probably due to high charge transfer resistance $(6,492 \Omega)$ [193]. Even though Mn can behave as an active site for ORR, metal oxides are well known to have poor electric conductivity; hence, this very high charge transfer resistance of the order of kilo-ohms. A superior way to use these materials for oxygen electrocatalysis would be to synthesize metal oxide nanoparticles and disperse them on a conductive powder, such as with Pt/C [156].

Hierarchical $\mathrm{NiCo}_{2} \mathrm{O}_{4}$ was synthesized by using a multistep process including electrospinning, sintering and co-precipitation [145]. Low content of transition metals (5\% Ni and19\% Co) achieved a preferable ORR mechanism towards the 4-electron pathway with an efficient electron transfer number of 3.91 and low onset and half-wave potentials of -0.17 and $-0.21 \mathrm{~V}$ vs. $\mathrm{Ag} / \mathrm{AgCl}$, respectively, in alkaline conditions. Stability shows a retained current density of $95 \%$ after 50,000 s according to chrono-amperometric testing. This electrospun material shows fast kinetics with a half-wave potential very close to its onset potential $(4 \mathrm{mV})$, leading to ORR mainly controlled by mass transport of the species. Electrocatalytic activity could likely be improved in the future by increasing porosity and surface area of the material (currently 60 $\mathrm{m}^{2} \cdot \mathrm{g}^{-1}$ ) using a soft-templating approach.

In general, many electrospun materials for ORR applications containing transition metals and heteroatoms have been synthesized over the last few years (See Table S.1 Section B). Key results exhibit a potential application in fuel cells by improving stability and tackling the cost of Pt-based cathodes. Fig. 12 shows a key parameter comparison between Pt-free materials in alkaline and acidic conditions. As seen in Fig. 12(a-c), most of these materials exhibit an onset potential above $0.9 \mathrm{~V}$ vs. RHE and half-wave potential above $0.8 \mathrm{~V}$ vs. RHE, fast kinetics and desirable performance, regardless of electrolyte $\mathrm{pH}$. Nonetheless, Fig. 12(d and e) show high stability after ADT (above 5,000 cycles) and chrono-amperometric testing ( $t>30,000 \mathrm{~s}$ ) of only a few of these materials. Unfortunately, few of these electrospun electrocatalytic materials are stable under ORR conditions for extended periods, or their activity is not demonstrated beyond many cycles or extended timescale by the authors. 
(a)

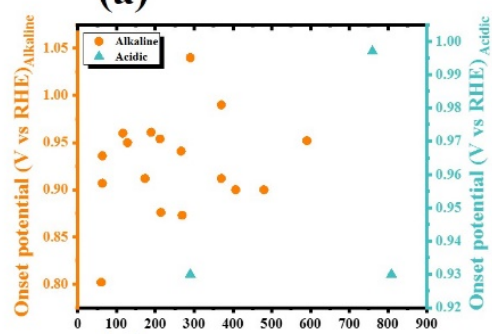

(d) Surface Area $\left(\mathbf{m}^{2} \mathbf{g}^{1}\right)$

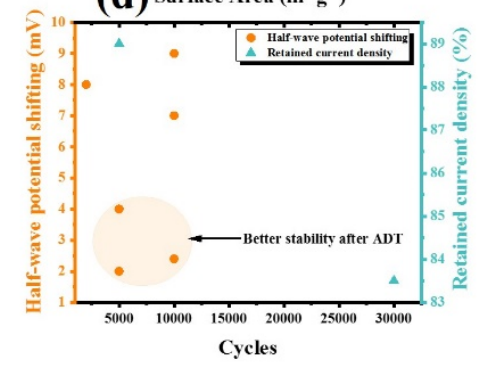

(b)

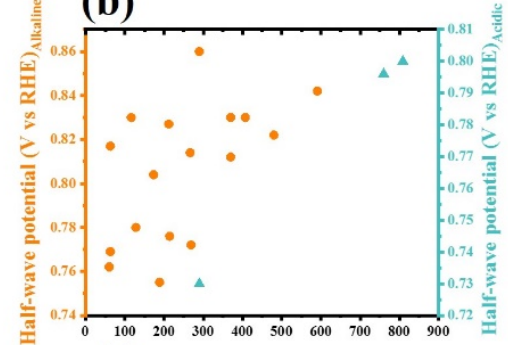

(e) Surface area $\left(\mathrm{m}^{2} \mathrm{~g}^{-1}\right)$

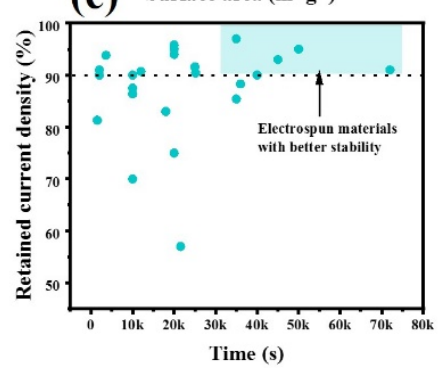

(c)

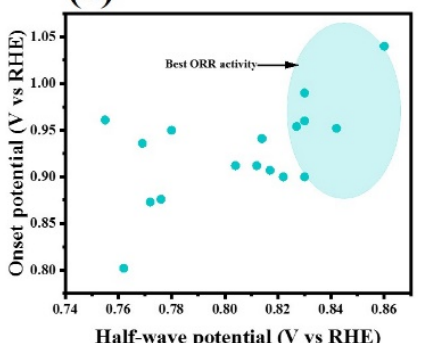

Fig. 12. Pt-free self-supported electrospun materials (a) surface area vs. onset potential comparison, (b) surface area vs. half-wave potential (alkaline and acidic) comparison, (c) half-wave vs. onset potential (alkaline) comparison, (d) halfwave potential shifting after ADT comparison and (e) retained current density after chrono-amperometric testing comparison. Testing in alkaline and acid conditions is differentiated using circle and triangle symbols, respectively.

Nevertheless, it should be taken into under consideration that few of these electrospun materials have been tested in actual acidic or alkaline fuel cells, with the majority of this data coming from half-cell measurements. Performance of fuel cell materials in operating devices can differ drastically to rotating disk electrochemical tests due to mass transport and contact resistance considerations [192], and so caution should be used when novel materials demonstrate good performance in half-cell testing. Full fuel cell testing is recommended to fully evaluate the performance of Pt-free catalysts for the ORR; however, it is hoped that at least some of the promising materials reviewed here will translate into technological PEMFCs. Additionally, the flexibility of the electrospinning approach in allowing tuneable microstructural properties in tandem with chemical control means that it is entirely feasible that electrospun catalysts could be further optimised for the operating environment of fuel cells in the near future. This highlights a key benefit of electrospinning over other synthesis routes towards Pt-free 3D catalysts [192].

Finally, fully metal-free electrospun materials emerge as another plausible option to substitute Pt in fuel cell cathodes[162-164]. Mainly, nitrogen-doped C porous nanofibres have been reported during recent years[162,163].

Recently, multiple heteroatom doped $\mathrm{C}$ nanofibres with good stability and electrocatalytic activity have attracted attention due to their facile few-steps synthesis [9]. A very interesting approach using electrospinning for production of $\mathrm{N}$-doped carbons on $\mathrm{TiO}_{2}$ nanofibers was 
reported by Hassen et al. [204]. This simple electrospinning and thermal processing achieved high surface area due to dominant mesoporosity $\left(188.6 \mathrm{~m}^{2} \cdot \mathrm{g}^{-1}\right)$, high $\mathrm{N}$ content $(8 \%)$ in the sample and a retained current density higher than platinum after ADT (83.5\% after 30,000 cycles) [204].

A very interesting and novel example of metal-free electrospun fibres for ORR was published by Gong et al. [164]. Nitrogen and fluorine co-doped carbon nanofibres were synthesised via a facile electrospinning method and subsequent thermal treatments. The nanofibers obtained showed an outstanding surface area of $709.78 \mathrm{~m}^{2} \cdot \mathrm{g}^{-1}$, with $2.06 \% \mathrm{~N}$ and $2.81 \% \mathrm{~F}$ content. This metal-free electrocatalyst showed good electrocatalytic activity in acidic and alkaline conditions (onset potential 0.94 (alkaline) and 0.635 (acidic) V vs. RHE, half-wave potential 0.81 (alkaline) and 0.257 (acidic) V vs. RHE, diffusion-limited current density 4.9 (alkaline) and 4 (acidic) $\mathrm{mA} \cdot \mathrm{cm}^{-2}$, electron transferred number 3.75 (alkaline) and 4 (acidic) [164].

In Fig. 13( $\mathrm{a}$ and $\mathrm{b}$ ), inherent porosity of the N, F co-doped $\mathrm{C}$ fibres can be distinguished by BET measurements. This characteristic provides accessibility for $\mathrm{O}_{2}$ to reach N-C and F-C active sites [164]. Fig. 13(c and d) show TEM micrographs of these fibres exhibiting amorphous and graphitic domains. Electrocatalytic performance and efficiency are shown in Fig. 13(e to g), demonstrating comparable LSV curves with Pt/C standard and the preferred 4electron pathway with low $\mathrm{H}_{2} \mathrm{O}_{2}$ yield. A high $\mathrm{H}_{2} \mathrm{O}_{2}$ indicates the prevalence of the twoelectron pathway; however, it should be noted that the thickness of the film used in RDE testing can mask the true production of peroxide intermediates and so this should always be taken into account when assessing the selectivity of such materials for the four-electron pathway [205,206]. Stability after $10,000 \mathrm{~s}$ is superior to $\mathrm{Pt} / \mathrm{C}$ standard, with a retained current density of $95.7 \%$ [164]. Differences in electronegativity between $\mathrm{C}, \mathrm{N}$ and $\mathrm{F}$ might promote electron density redistribution on carbons surrounding pyridinic and graphitic $\mathrm{N}$ and $\mathrm{F}$ and, as a consequence, generating Lewis acidic sites where $\mathrm{O}_{2}$ absorbs and desorbs with an efficient electron transference from these sites. 


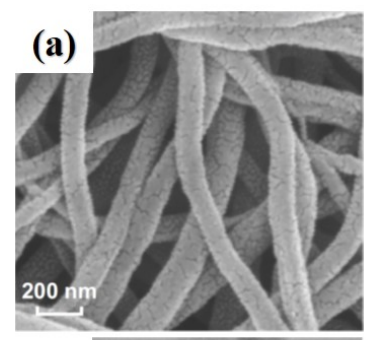

(c)

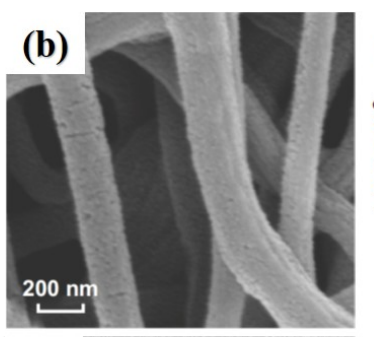

(d)
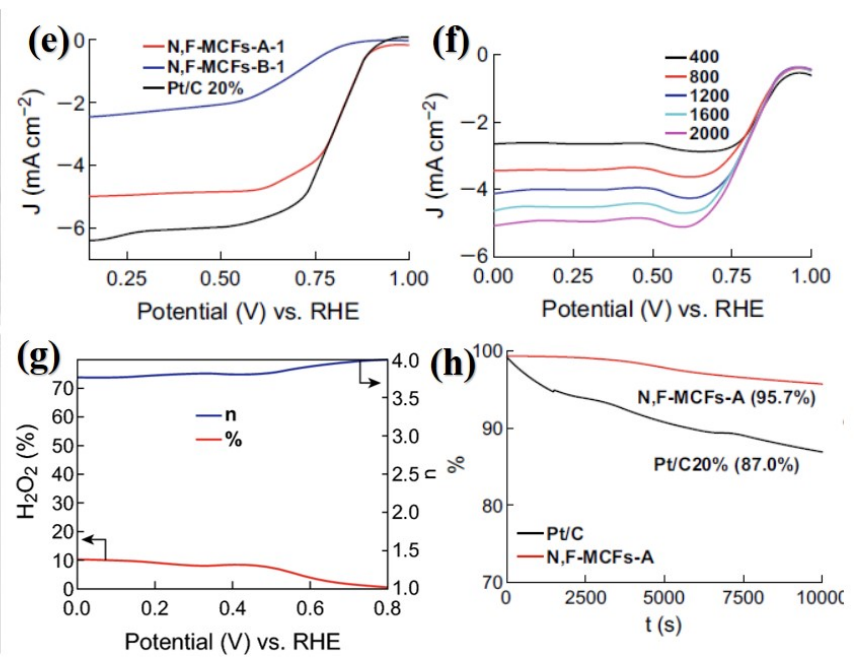

Fig. 13. (a and b) SEM micrographs of N/F co-doped nanofibres, (c and d) TEM micrographs, (e) LSV under oxygen reduction reaction conditions for N/F co-doped nanofibers and Pt/C, (f) LSV at different rotation speeds for KouteckýLevich analysis, $(\mathrm{g})$ electron transfer number vs. $\mathrm{H}_{2} \mathrm{O}_{2}$ yield at different applied potentials and (h) current-time $(i$ vs. $t)$ chrono-amperometric response for stability testing. Reproduced from Ref. [164] with permission from Springer Nature.

These reports show that metal-free electrospun materials should be considered as potential materials for substituting Pt in fuel cell cathodes, and more testing and understanding of performance in an actual fuel cell should be carried out in future investigations.

\subsection{Conclusions and outlook}

During recent years of research on the potential application of electrospun materials for ORR in cathodes of fuel cells, efforts have focused on developing porous and conductive materials that can provide Pt of higher stability and oxygen accessibility to Pt active sites. Great improvement in these parameters have been achieved, leading to increased durability and, hence, potentially a reduction in the cost of maintenance or substitution of cathodes in operating fuel cell MEAs.

A huge effort has been invested in developing transition metal electrospun nanofibres as a lower-cost and industrially scalable option. Unfortunately, these materials usually are not tested in a fuel cell setup and most of the data correspond to half-cell measurements in acidic or alkaline conditions. Metal-free electrospun materials are currently underdeveloped for actual fuel cell applications, largely because of the focus of the oxygen catalysis field towards other fabrication techniques of heteroatom-doped carbons for these purposes. Nonetheless, transition metal and heteroatom doped electrospun materials show good ORR activity, and broader knowledge should be developed for their application in devices to optimize parameters in an actual fuel cell (catalyst mass loading, free-standing MEA assembly, ink formulation, electrocatalytic surface area, etc.) and standardized procedures for testing Pt-free materials in 
them should be developed, noting the common issues with testing in a half-cell environment $[205,206]$.

In general, electrospinning should be considered as a promising option for mass production of cathodes for low-temperature fuel cells. Extra benefits of this manufacturing process of high surface area and conductive nanomaterials is the ability to produce free-standing electrodes that could act simultaneously as a GDL with tuneable microstructural properties and a selfsupported cathode for these energy devices.

\section{Metal-air batteries and beyond}

\subsection{Introduction}

In the early 2000s, research in electrospun carbon fibres was primarily concerned with understanding the critical operating parameters, characterizing mechanical properties and exploring the morphology of carbon in the fibres [207]. The high surface area of electrospun carbon fibres piqued interest in using the material as a hydrogen storage structure, and work activating the surface of the fibres with $\mathrm{KOH}, \mathrm{NaOH}$, and $\mathrm{K}_{2} \mathrm{CO}_{3}$ soon followed [208]. Techniques to produce hollow, porous, and ribbon-like fibres were developed, as well as composite fibres of polymer blends. Fibres with electrochemical activity have been created by incorporating metal salts in the electrospinning solution. The inclusion of these compounds has been shown to increase porosity, with pore space forming as metal particles are produced during pyrolysis [209].

Recently, research efforts have explored integrating catalytically active fibres into metal-air batteries. Metal-air batteries are electrochemical cells that produce electricity by oxygen reduction at the cathode and metal oxidation at the anode. Atmospheric oxygen diffuses into the cell through a porous gas diffusion layer, reaching active sites in the catalyst layer where the oxygen reduction reaction proceeds. Their high energy density and low cost make metalair batteries attractive options for energy storage. As the oxygen supply from the air is effectively infinite, the capacity of a metal air battery is determined by the amount of anodic metal in the cell. Lithium and zinc are the most developed metal-air battery technologies. Zincair batteries utilize an alkaline aqueous electrolyte, typically $\mathrm{KOH}$. The reactivity of lithium makes electrolyte design in lithium-air batteries more complicated. Some of the challenges in developing metal-air batteries are expensive catalysts such as platinum and iridium, electrolyte degradation, and passivation of catalyst and anode materials as discharge products accumulate on the surfaces [210]. 
Research in applying electrospun materials to lithium- and zinc-air batteries is primarily focused on fibres employing transition metals and/or nitrogen-carbon heteroatoms embedded on the fibre surface to replace the conventional precious metal catalyst layer on the cathode. Different transition metals, such as cobalt, nickel, and lanthanum have been explored, as well as nitrogen-doped fibres. A common observation throughout this research has been that catalytic activity increases with the amount of surface area (i.e., the number of active sites available) for all catalytic materials explored, which is expected. In addition to their use as catalyst materials, electrospun fibres are being investigated as gas diffusion layers for metalair batteries, taking advantage of their open-pore structure. The ability to electrospin different polymers has prompted research in diffusion layers composed of functional fibres.

\subsection{General overview of electrospun material production}

There is a great deal of work exploring the efficacy of metal-doped carbon nanofibres as catalyst/catalyst support materials for metal-air batteries [211-216]. Production of these fibres is relatively straightforward; a homogeneous solution of polyacrylonitrile (PAN) and transition metal salts (e.g., hydrated cobalt acetate, iron(III) acetylacetonate) dissolved in DMF is extruded from a needle to a collector, producing a sheet of uncarbonized PAN fibres with metal salts distributed throughout the fibre's volume. Other compounds may be added to the electrospinning solution, such as cellulose acetate [211] or [3-(2aminoethylamino)propyl]trimethoxysilane (AEAPT), a ligand [212], to change fibre morphology or metal particle distribution, respectively. After spinning, fibres are carbonized in a multi-stage fashion. The first step, a "stabilization stage" between $220{ }^{\circ} \mathrm{C}$ and $280{ }^{\circ} \mathrm{C}$ for 1 to $4.5 \mathrm{hrs}$, and a second carbonisation step pyrolyzing fibres at $800-1,000^{\circ} \mathrm{C}$ under an inert atmosphere of $\mathrm{N}_{2}$ or Ar. Heating rates are variable, between 1 and $5^{\circ} \mathrm{C} \cdot \mathrm{s}^{-1}$. Though not common, carbon fibres can be produced from PAN with one high-temperature treatment under an inert atmosphere, forgoing the stabilization stage [216].

These carbonisation/pyrolysis techniques produce carbon fibres with high purity and an amorphous carbon core surrounded by a shell of graphitic carbon. The inclusion of metal salts in uncarbonized PAN fibres produces carbon fibres with metal particles embedded in the fibre surface and fibre bulk. During the high-temperature pyrolysis step, metal precursors decompose, creating pore space and producing fibres with higher specific surface area than solid carbon fibres produced from pure PAN. High surface area materials have been shown to more effectively catalyse reactions at the cathode due to their greater number of active sites for 
reactants. Embedding transition metals and metal oxides in carbon fibres creates a catalytically active material with improved conductivity [216].

Although mats of carbon fibres produced from electrospun PAN form free-standing sheets, research in catalytically active electrospun materials for metal-air battery cathodes often involves grinding the carbon fibres, distributing the particles in a solvent with a binder component (e.g., PTFE or Kynar 761) and applying them to an electrode surface with either a doctor blade or spraying technique to create a catalyst layer [212,217]. Unless otherwise mentioned, this is the technique used to test the electrochemical performance of the electrospun materials reviewed in this work $[212,214,216]$.

\subsection{Common characterization techniques for electrospun materials}

As more research investigating electrospun materials for metal-air battery applications has been published, a standard set of characterization techniques have emerged. XRD patterns are useful in determining the morphology of the carbon in the fibres [211-213,215]. A broad peak at $26^{\circ}$ corresponds to the (002) plane of graphitic carbon composing the outer layer of the electrospun carbon fibres. Not only is a graphitic layer of carbon formed during carbonisation, but the inclusion of metal particles has also been shown to catalyse the formation of graphite [218]. This was demonstrated by Martinez Crespiera et al. in their work developing Pd-doped carbon fibres for Li-air batteries [212]. XRD patterns of Pd-doped fibres with poor Pd distribution did not feature a strong graphitic carbon peak. Peaks from the metals and metal compounds present in the XRD patterns are useful in confirming the components present in the final carbon fibre product. The ability to identify and differentiate between $\mathrm{MnS}$ and $\mathrm{MnO}$ peaks on XRD patterns allowed Wang et al. to infer that sulfur in the thiourea incorporated into the electrospinning solution reacted with $\mathrm{Mn}$, resulting in a final product containing $\mathrm{MnS}$ rather than $\mathrm{MnO}$ [216]. HRTEM images of the fibres revealed Mn and S signal at the same location, and no relationship between carbon and sulfur, indicating fibres contain primarily $\mathrm{MnS}$ rather than sulfur-carbon complexes [216].

X-ray photoelectron spectroscopy (XPS) is often coupled with XRD in compositional analysis of doped electrospun carbon fibres, particularly to gain insight into the heteroatoms created during pyrolysis [213-216]. Using XPS to analyse cobalt and cobalt oxide doped fibres, Hyun and Shanmugam were able to identify the various forms of cobalt in the final product, as well as the specific N-C heteroatoms formed [215]. XPS spectra illustrated that graphitic-N and pyridinic-N are the two common heteroatoms present in their final product, an important result as they have been shown to enhance the limiting current density and improve the onset potential 
of the oxygen reduction reaction, respectively [215,219,220]. This result is repeated in Fe- and $\mathrm{Ni}$-doped fibres, as presented in the research of $\mathrm{Ji}$ et al. [211]. The shallow depth of investigation inherent to XPS is also useful in characterizing doped carbon fibres. As Huang et al. point out, the transition metals used to produce electrochemically active electrospun carbon fibres tend to oxidize, but the presence of signals from the pure metals on the XPS spectra in addition to peaks from metal oxides, allows one to infer that the particles may have a metal oxide shell and that the shell is either incomplete or only a few nanometres thick [213].

A shared finding from the research in metal-doped electrospun carbon fibres is an increase in specific surface area due to the formation of pores as precursor metal salts decompose during pyrolysis [212,215]. BET analysis of $\mathrm{N}_{2}$ adsorption isotherms is commonly employed to estimate specific surface area, and qualitative analysis of adsorption isotherms is useful in identifying mesoporous materials. Solid electrospun carbon fibre materials contain macropores, supported by the Type 1 adsorption isotherms they exhibit. The inclusion of pore space in the fibres is apparent in their Type 4 adsorption isotherms that typically feature hysteresis, as well as their increased BET specific surface area [213]. Ji et al. have produced high surface area electrospun carbon fibre materials by growing carbon nanotubes from sites on the surface of the spun carbon fibres [211]. Higher porosity materials tend to facilitate oxygen dissociation to reaction sites, feature more sites for surface catalysed reactions, and have a higher capacity for discharge products that form on fibre surfaces [215].

There is a common set of electrochemical performance metrics used to evaluate metal-air cells. The onset potentials of the ORR that occurs during discharge and the oxygen evolution reaction (OER) when the cell is charged represent the voltages where the thermodynamic and kinetic barriers preventing the reactions are overcome, and a sharp increase in current is observed. In practice, ORR and OER onset potentials are lower and higher than the theoretical redox potentials of oxygen, respectively. It is desirable to maximize ORR onset potential, correlating to a higher operating voltage, and a minimize OER, meaning that cell charging is less inhibited by side reactions or discharge products. Charge and discharge potentials are important metrics and are similar in concept to onset potentials. The discharge potential is the voltage at which a cell spontaneously discharges for some current density, while the charge potential is the voltage required to promote the discharge products from their lower energy state. The difference between the two is referred to as the voltage gap, and minimization of this gap indicates better operating efficiency. The efficiency of cell operation is further characterized by the round-trip efficiency and the Coulombic efficiency metrics. Round-trip efficiency is the ratio of energy 
released in a discharge cycle to the energy input during a charge cycle. Coulombic efficiency is the ratio of discharge and charge capacity; the significance of Coulombic efficiency is illustrated in cycling experiments. As a cell undergoes a series of charge and discharge cycles losses attributed to coulombic efficiency compound, and the capacity retention of the cell after the final cycle may calculated by raising the coulombic efficiency to the $n$th power, where $n$ is the number of cycles the cell has undergone.

\subsection{Electrochemically active electrospun fibres: Li-Air}

Work in integrating electrospun fibres doped with metals and metal oxides in lithium-air cells has resulted in higher specific discharge capacity, improved round-trip efficiency, and better cycling capabilities than unmodified electrospun carbon fibres, demonstrating their great potential as cathodes in metal-air batteries. CV experiments demonstrate higher limiting current density for ORR and OER reactions than unmodified ECNFs, and onset potentials comparable to $\mathrm{Pt} / \mathrm{C}$ and $\mathrm{Ir} / \mathrm{C}$, respectively. The electrochemical advantages of doped electrospun carbon fibres vary depending upon the doping metals and fibre characteristics such as specific surface area and the presence of heteroatoms. Differences in the electrochemical evaluation parameters make direct comparison across published research difficult.

Martinez Crespiera et al. developed an electrospun carbon fibre material doped with palladium to serve as a cathodic catalyst layer in a lithium-air battery. Their electrospinning process was similar to others outlined in Section 5.2, incorporating a $\mathrm{Pd}$ salt $\left(\mathrm{Pd}\left(\mathrm{Ch}_{3} \mathrm{COO}\right)_{2}\right)$ into the PAN/DMF solution, electrospinning the solution, and carbonizing the composite PAN fibres in a furnace under a $\mathrm{N}_{2}$ atmosphere, serving as a 'one-step' combination and formation of catalyst and support, as opposed to methods that first synthesise the catalyst particles, and then immobilise them on their carbon supports. In one sample a ligand that associates with $\mathrm{Pd}^{2+}$ ions was incorporated into the electrospinning solution and ultimately improved $\mathrm{Pd}$ particle distribution. Using XRD, the fibres were shown to contain Pd particles with a face centred cubic crystalline structure. Pd composite fibres exhibited higher specific surface area that increased with Pd acetate concentration. The pore size, however, did not scale with Pd concentration; it remained constant with an average pore size of $3.6 \mathrm{~nm}$ [212].

Electrochemical testing showed that the incorporation of Pd did not necessarily improve the discharge capacity, only the fibres with $7.06 \mathrm{wt} \% \mathrm{Pd}$ had a higher discharge capacity than the bare carbon fibres. Pd particles did improve the Coulombic efficiency of the cathode material, increasing from $56 \%$ for bare carbon fibres to $95 \%$ for the $7 \mathrm{wt} \% \mathrm{Pd}$-doped carbon fibres. This is attributed to $\mathrm{Pd}$ embedded in the fibres to facilitate the decomposition of $\mathrm{Li}_{2} \mathrm{O}_{2}$ and other 
side products created during discharge. SEM images following a discharge cycle showed that $\mathrm{Li}_{2} \mathrm{O}_{2}$ forms in small grains on Pd sites with a nanocrystalline morphology, an advantage when charging as it is more readily decomposed than amorphous $\mathrm{Li}_{2} \mathrm{O}_{2}$ [221]. The lower charge potential reduces charge overpotential, side reactions during charging, and the accumulation of carbonate products $\left(\mathrm{Li}_{2} \mathrm{CO}_{3}\right)$, leading to greater efficiency in the battery.

The advantage of Pd particles on the surface of the carbon fibres is further illustrated by the cycling experiments in this work. The Pd-doped fibres that used a ligand had the smallest $\mathrm{Pd}$ particle size and lowest particle loading, but the best longevity. Charge potential of this sample was kept below $3.5 \mathrm{~V}$ for the first 20 cycles, whereas all other materials required a potential above $4 \mathrm{~V}$. This too is attributed to the difference in morphology of $\mathrm{Li}_{2} \mathrm{O}_{2}$ that is formed on the cathode surface during discharge.

Hyun et al. investigated cobalt and cobalt oxide particles on nitrogen-doped carbon fibres as a cathodic catalyst for lithium-air batteries [215]. Again, the formation of particles during carbonisation produced a high specific surface area fibre. Their pristine N-ECNF materials had a specific surface area of $29.5 \mathrm{~m}^{2} \cdot \mathrm{g}^{-1}$, while cobalt-doped fibres (Co/N-CNR), and cobalt-cobalt oxide fibres (Co-CoO/N-CNR) had a specific surface area of 370 and $373 \mathrm{~m}^{2} \cdot \mathrm{g}^{-1}$, respectively. The nitrogen-doped carbon fibres with cobalt and cobalt oxide particles, $(\mathrm{Co}-\mathrm{CoO} / \mathrm{N}-\mathrm{CNR})$ exhibited the best electrochemical performance. $\mathrm{CV}$ indicated that this material was the best bifunctional catalyst they tested, the ORR peak current density being $\sim 37 \mathrm{~mA} \cdot \mathrm{cm}^{-2}$ and the OER being $-0.9 \mathrm{~mA} \cdot \mathrm{cm}^{-2}$. The Co-CoO/N-CNR materials also delivered the highest discharge capacity, $10,555 \mathrm{~mA} \cdot \mathrm{h} \cdot \mathrm{g}^{-1}$ at a current density of $100 \mathrm{~mA} \cdot \mathrm{g}^{-1}$, versus the $7,089 \mathrm{~mA} \cdot \mathrm{h} \cdot \mathrm{g}^{-1}$ of the $\mathrm{N}-\mathrm{CNR}$ fibres. These fibres also had the highest discharge capacity as current density increased, the best cycle stability, and a round trip efficiency of $73.6 \%$. The cycle stability of the Co$\mathrm{CoO} / \mathrm{N}-\mathrm{CNR}$ materials is higher than the $\mathrm{Co} / \mathrm{N}-\mathrm{CNR}$ material, indicating that the presence of $\mathrm{Co}$ and $\mathrm{CoO}$ particles together creates better bifunctional catalysts than Co particles alone. High rate capability is attributed to the improved oxygen diffusion and electrolyte saturation offered by the electrospun catalytic material, as well as the high specific surface area of the doped materials [215].

Post-mortem analysis of the air cathode materials after cycling experiments illustrated why carbon fibres with cobalt and cobalt oxide performed the best. The pristine N-CNR material had an accumulation of $\mathrm{Li}_{2} \mathrm{CO}_{3}$ and $\mathrm{Li}_{2} \mathrm{O}_{2}$, covering catalytic sites on the carbon surface. $\mathrm{Co}$ and $\mathrm{CoO}$ particles in the $\mathrm{Co}-\mathrm{CoO} / \mathrm{N}-\mathrm{CNR}$ material acted as nucleation sites for $\mathrm{Li}_{2} \mathrm{O}_{2}$, delaying 
the onset of complete surface coverage during discharge. This result has been replicated in other work investigating Co doped carbon fibers as air cathode materials, and is significant as the ability to continuously form and decompose $\mathrm{Li}_{2} \mathrm{O}_{2}$ improves rate capability and cycle stability [222]. $\mathrm{Li}_{2} \mathrm{O}_{2}$ has a higher energy of adsorption onto $\mathrm{Co}$ and $\mathrm{CoO}$ than carbon, limiting the formation of $\mathrm{Li}_{2} \mathrm{CO}_{3}$ in the doped fibres, which serves to improve cycling performance and limit parasitic side reactions. $\mathrm{Li}_{2} \mathrm{CO}_{3}$ is formed at the electrolyte - carbon fibre interface where $\mathrm{Li}_{2} \mathrm{O}_{2}$ is present (Fig. 14).

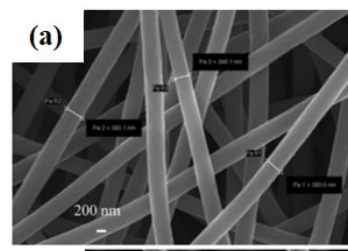

(c)

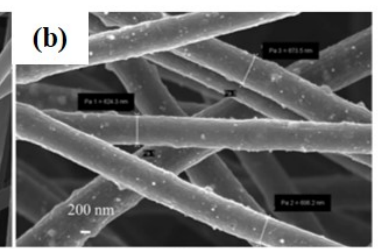

(d)

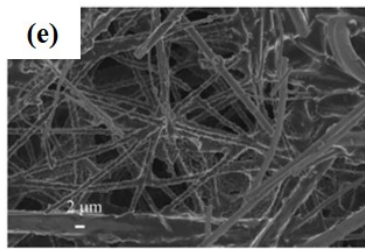

(g)

(f)

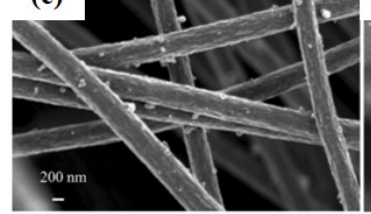

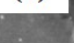
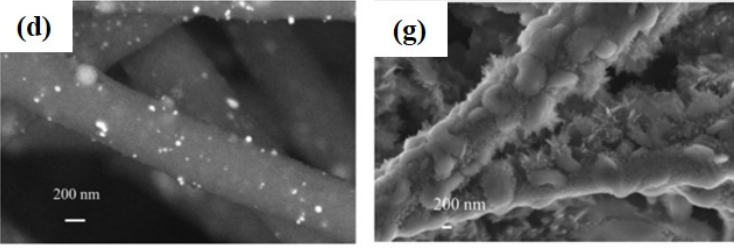

(1)
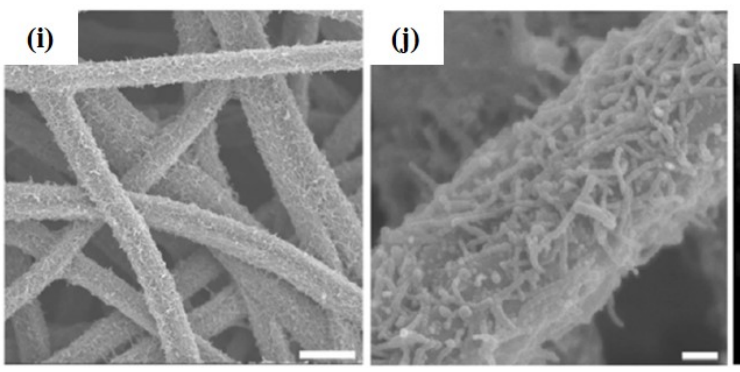

(k)

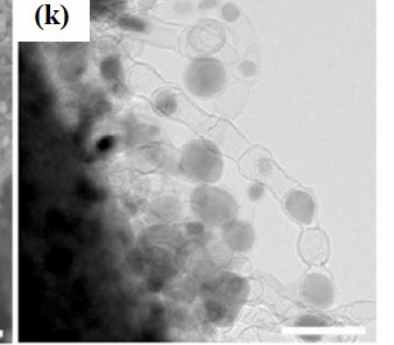

Fig. 14. Examples of catalytically active electrospun fibres produced for lithium-air batteries. (a-d) and (e-h), SEM images of electrospun carbon fibres with Co particles, and the accumulation of $\mathrm{Li}_{2} \mathrm{O}_{2}$ on the Co during discharge, respectively. Reproduced from Ref [222] with permission from Springer Nature. Fibre diameters in (a) are indicted as roughly $350-400 \mathrm{~nm}$ and in (b) $600-675 \mathrm{~nm}$. (i and j) SEM images, and (k) TEM image of electrospun carbon fibres with peapod-like carbon nanostructures on the fibre surface. Scale bars for (i) to (k): (i) $1 \mu \mathrm{m}$, (j) $100 \mathrm{~nm}$ and (k) $50 \mathrm{~nm}$. Reproduced from Ref [211] with permission from American Chemical Society.

Rather than increase the number of active sites through porosity developed within electrospun metal-carbon fibres during pyrolysis, Ji et al. produced electrospun carbon materials with embedded metal particles and peapod-like carbon nanotube structures on the fibre surfaces [211] (Fig. 14). This hierarchical architecture was formed during the carbonisation process. The electrospinning solution consisted of PAN, cellulose acetate and iron (III) acetylacetonate in DMF. The electrospinning process was standard, and materials were carbonised with melamine under a nitrogen atmosphere; melamine served to create nitrogen-doped carbon fibres [223]. The highest performing final product, CNT-CNFF-800, was shown to be quite flexible with diffusion properties exceeding the existing $\mathrm{Pt} / \mathrm{C}$ and Ni-foam materials used as controls. Intentional formation of peapod like carbon structures on the fibre surface increased the specific surface area of the material; BET analysis found the surface area to be $220.24 \mathrm{~m}^{2} \cdot \mathrm{g}^{-}$ 
1. The electrospun materials were utilized as a free-standing electrode structure without incorporating a binder material.

The electrode materials were then incorporated into a lithium-air battery and performance was compared to a similar cell with a conventional $\mathrm{Pt} / \mathrm{C}$ cathode catalyst. The difference between charge and discharge voltages of CNT-CNFF-800 was $0.15 \mathrm{~V}$, compared to $0.43 \mathrm{~V}$ for the Pt/C materials, demonstrating the bifunctional capabilities of CNT-CNFF-800, catalysing both the ORR and OER reactions. The CNT-CNFF- 800 cell operated at a high voltage, $\sim 3.4 \mathrm{~V}$ for more than $120 \mathrm{hr}$ in open air [211].

Charge-discharge experiments showed that the CNT-CNFF-800 material is capable of relatively high current charge and discharge operation, and that the material catalysed the reduction reaction via the 4-electron pathway. Tafel plots of CNT-CNFF-800 and Pt/C had similar slopes, confirming that the two materials catalyse the ORR along a similar pathway. Cycling performance did decrease over time, after 21 cycles the overpotential had reached a value of $0.3 \mathrm{~V}$. The authors attribute this to the formation of $\mathrm{Li}_{2} \mathrm{CO}_{3}$, and catholyte loss from the cell. The air cathode material in this work is unique in that it was a free-standing fibre mat that functions on a level comparable to conventional $\mathrm{Pt} / \mathrm{C}$ catalysts. The ability to use an electrospun material as a cathode layer not only simplifies processing, but also highlights the ability of electrospinning techniques to produce porous materials that can be directly integrated into metal-air cells.

\subsection{Electrochemically active electrospun fibres: $\mathrm{Zn}$ - Air}

Composite electrospun fibres have demonstrated potential as replacements for high-cost platinum group metal (PGM) catalysts. Metal particles embedded in the fibre surface and fibres containing nitrogen-carbon have promising catalytic performance. Results illustrate that high surface area of the catalytically active fibres is critical, as fibres with embedded metal compounds outperform powders of the same material. Several of the materials discussed in this section are flexible fibre sheets, an uncommon characteristic in carbon fibres produced via pyrolysis. Additionally, several electrospun materials have been integrated into zinc-air cells as free-standing fibre sheets, an elegant alternative to catalyst layers composed of ground fibres and binder material.

Manganese sulfide and metallic cobalt embedded nitrogen-doped carbon nanorods (CMS/NCNF) have been developed as a catalyst for zinc-air batteries by Wang et al. [216]. Cobalt acetate and manganese acetate were included in the PAN/DMF electrospinning solution. 
Sulfur was provided by thiourea in the solution; XRD and scanning transmission electron microscopy (STEM) showed that rather than form S-C heteroatoms, the sulfur associated with $\mathrm{Mn}$ in forming $\mathrm{MnS}$. Mn embedded fibres without sulfur (CMO/NCNF) were produced for comparison, as were $\mathrm{Mn}$ embedded fibres without nitrogen-doping (CMO/CNF). Fibres containing sulfur were found to have a higher ORR onset potential than those without, and fibres without nitrogen doping or manganese performed worst of all, as no carbon-nitrogen heteroatoms were present to catalyse the reaction. A unique physical property of the electrospun catalyst was its flexibility; performance was maintained at high angle bending, leading to potential application in wearable or flexible devices.

$\mathrm{CV}$ experiments showed that the CMS/NCNF catalyst layer had similar performance to conventional $\mathrm{Pt} / \mathrm{C}$ catalysts, with an ORR onset potential of $0.969 \mathrm{~V}$ compared to 0.989 for $\mathrm{Pt} / \mathrm{C}$. The limiting current density of CMS/NCNF was slightly higher than $\mathrm{Pt} / \mathrm{C}, 4.43 \mathrm{~mA} \cdot \mathrm{cm}^{-}$ ${ }^{2} \mathrm{v} 4.26$, respectively. Of the novel catalyst materials produced, CMS/NCNF showed the best performance, indicating that the $\mathrm{MnS}$ particles possess better ORR catalytic performance. The electron transfer number for the CMS/NCNF catalyst was found to be 4 at potentials between 0.5 and $0.65 \mathrm{~V}$. The CMS/NCNF material outperformed conventional Pt/C catalyst in cycle testing, after over $100 \mathrm{hrs}$ of 20-minute charge and 20-minute discharge cycles there were minimal voltage losses in the $\mathrm{CMS} / \mathrm{NCNF}$ material. The conventional $\mathrm{Pt} / \mathrm{C}$ catalyst was much less stable, lasting for less than 45 hours.

Electrospun $\mathrm{LaCoO}_{3}$ embedded carbon fibres have been explored as cathode catalyst materials in zinc-air batteries. The work showed that embedded metal compounds in electrospun carbon fibres can have better catalytic activity than powder-based catalysts composed of the same metal compounds, and the authors attribute this to a higher specific surface area and a larger number of active sites. As has been shown in other work, the formation of metal compounds during pyrolysis creates a final fibre product with higher specific surface area and metal or metal oxide inclusions [214].

The $\mathrm{LaCoO}_{3}$ materials did not outperform the conventional precious metal catalyst used as a reference. The PtRu/C catalyst material had an $n$ value of 4.08 for the ORR, while the $\mathrm{LaCoO}_{3}$ fibre $n$ value was 3.43, indicating that the $\mathrm{LaCoO}_{3}$ does not catalyse the ORR along the same ideal four-electron pathway as $\mathrm{Pt} / \mathrm{C}$. The different catalyst loading of the materials developed in this work makes a direct comparison of electrochemical properties difficult. Tafel plots showed that ORR on the $\mathrm{LaCoO}_{3}$ carbon fibre materials had faster kinetics than the 
conventional PGM catalyst used. The higher conversion rate is attributed to the higher surface area and greater number of active sites in the $\mathrm{LaCoO}_{3}$ materials.

Nitrogen-doped electrospun carbon fibres without embedded metals, are also being investigated as cathode materials for zinc-air batteries. Rather than electrospin PAN, Lui et al. spun polyimide (PI) and carbonized the fibres between 900 and $1,100{ }^{\circ} \mathrm{C}$ under an $\mathrm{Ar}$ atmosphere [224]. The result was a flexible mat of carbon fibres; unlike PAN-derived carbon fibres, which have a core/shell structure of amorphous and graphitic carbon. HRTEM showed that the PI derived fibres consisted of mostly amorphous carbon speckled with graphitic regions (Fig. 15). The fibres had a large specific surface area $1,249 \mathrm{~m}^{2} \cdot \mathrm{g}^{-1}$ and electrical conductivity of $147 \mathrm{~S} \cdot \mathrm{m}^{-1}$, significantly lower than the conductivity of carbon fibre mats of electrospun PAN. The authors mention that nitrogen compounds formed during the pyrolysis of $\mathrm{PI}, \mathrm{NH}_{3}$ and $\mathrm{HCN}$ for example, act as doping agents and that increasing the residence time of furnace gas increased nitrogen doping.
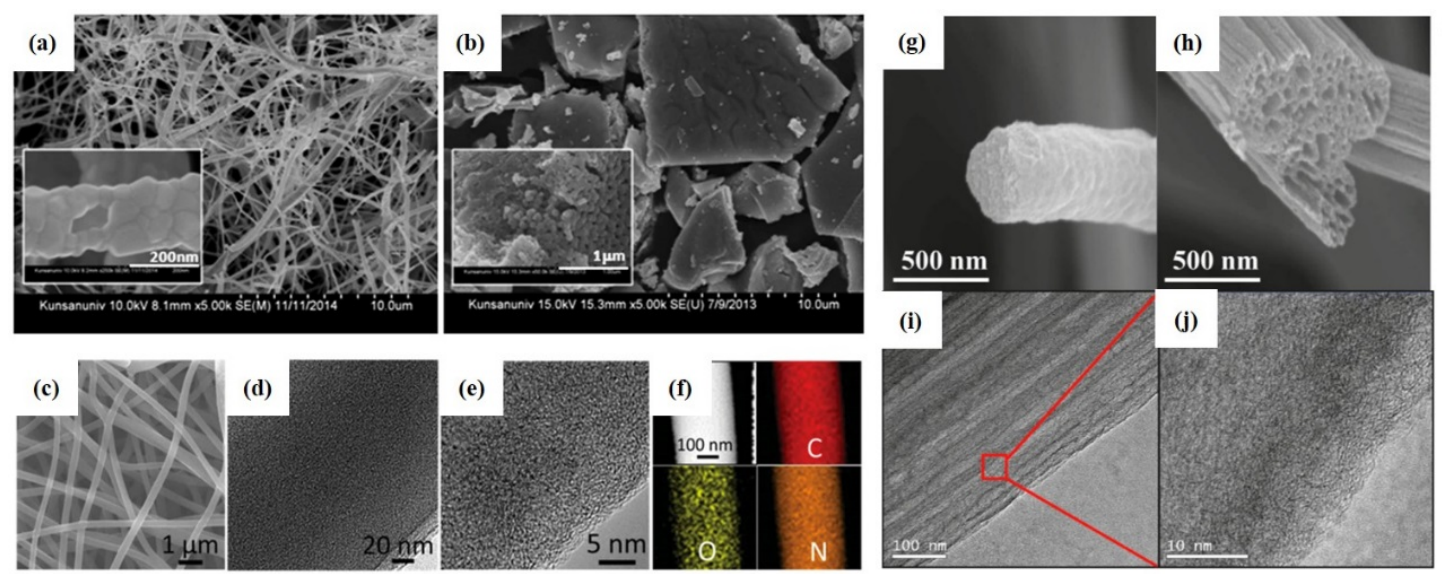

Fig. 15. Examples of electrospun fibres produced for zinc-air flow batteries. (a and b), The advantage of supporting $\mathrm{LaCoO}_{3}$ on electrospun carbon fibres (a) rather than producing a porous $\mathrm{LaCoO}_{3}$ material (b) (insets: HRSEM images of samples). Reproduced from Ref. [214] with permission from Springer Nature. (c) SEM image of electrospun carbon fibres produced from polyimide (PI); ( $\mathrm{d}$ and e), HRTEM images of the same fibre; (f) STEM image showing the elemental mapping in the fibre. Reproduced from Ref. [224] with permission from John Wiley and Sons. (g to j) Illustrates the 'churros' morphology fibres; $(\mathrm{g})$ is the uncarbonized PAN/PS fibre and $(\mathrm{h})$ is post carbonisation [225].

The fibres produced contained graphitic, pyrrolic, and pyridinic N-graphene heteroatoms. Graphitic-N composition increased with carbonisation temperature, an expected result as pyrrolic and pyridinic $\mathrm{N}-\mathrm{C}$ moieties are less stable. The fibres carbonized at $1,000{ }^{\circ} \mathrm{C}(\mathrm{NCNF}-$ 1000) showed better performance than conventional Pt/C catalyst in a zinc-air cell. The NCNF1000 fibres had a small voltage gap, with a discharge potential of $1.2 \mathrm{~V}$ and a charge potential of $1.93 \mathrm{~V}$ at $10 \mathrm{~mA} \cdot \mathrm{cm}^{-2}$; the fibres were also exhibited good cycle stability, after 500 cycles the voltage gap increased by $0.13 \mathrm{~V}$, while the $\mathrm{Pt} / \mathrm{C}$ catalyst voltage gap increased by $0.38 \mathrm{~V}$ 
after cycling. The NCNF-1000 catalyst was shown to catalyse the ORR along a four-electron pathway, and the OER onset potential was lower than $\mathrm{Pt} / \mathrm{C}$.

In 2013 Park et al. explored metal-free electrospun carbon fibres with a porous "churro-like" structure as zinc-air catalysts [225]. The fibres were produced from a PAN/PS blend, with the PS acting as a sacrificial element that decomposed during pyrolysis, leaving void space in the carbon fibres. The inner pore structure of the fibres (Fig. 15) is interesting in that it is linear, along the carbon fibre's axis (hence the churro analogy); this is due to the difference in surface energy between PAN and PS. The formation of internal channels via electrospun a mixture of PAN and PS solution was also successfully formed with a higher concentration in the redox flow battery study, and coexist with micro-scaled bundle fibres [146]. While being electrospun, the difference in surface energy results in microphase separation. The specific surface area of the carbon fibres increased with pyrolysis temperature, reaching $1,271.2 \mathrm{~m}^{2} \cdot \mathrm{g}^{-1}$, determined by BET analysis of $\mathrm{N}_{2}$ adsorption. Additionally, as pyrolysis temperature increased, an improvement in the ORR onset potential and limiting current density was observed, the fibres carbonized at $1,100{ }^{\circ} \mathrm{C}$ (N-CFs-1100) were shown to have the best electrochemical performance of the materials produced.

Electrochemical performance of the fibres with a churro morphology was comparable to $\mathrm{Pt} / \mathrm{C}$ for the ORR reaction, OER performance was not presented in this work. The electron transfer number was found to be nearly $4(3.7-3.8)$ for the N-CFs-1100. By varying the current density between 0 and $340 \mathrm{~mA} \cdot \mathrm{cm}^{-2}$, Park et al. showed that the N-CFs-1100 had a similar peak power density to $\mathrm{Pt} / \mathrm{C}, 192 \mathrm{~mW} \cdot \mathrm{cm}^{-2}$ compared to $194 \mathrm{~mW} \cdot \mathrm{cm}^{-2}$ of the conventional catalyst. The electrochemical performance of the fibres is attributed to graphitic nitrogen groups formed during carbonisation. The increased surface area of the N-CFs-1100 makes more of the graphitic-N sites available, accounting for the improved ORR activity of the higher temperature pyrolysis.

\subsection{Other applications of electrospinning to M-Air cathodes}

In addition to improving the electrochemical performance of metal-air battery cathodes, electrospun materials are being investigated as solutions to other metal-air battery challenges. Zinc-air batteries use an alkaline electrolyte, typically aqueous $\mathrm{KOH}$. Carbon dioxide in the air reacts with the electrolyte, forming carbonates that reduce cell performance by reducing electrolyte conductivity, clogging pores, and decreasing active surface area. To limit the diffusion of $\mathrm{CO}_{2}$ to the electrolyte interface in the active layer, Huang et al. developed a polystyrene/polyethyleneimine (PS/PEI) composite electrospun membrane [226]. The amine 
groups in PEI polymers have been shown to be good low-temperature $\mathrm{CO}_{2}$ adsorbent materials.

The electrospinning process employed to produce the PS/PEI fibres was straightforward, the two polymers were dissolved in DMF, producing a homogeneous solution that was then electrospun. The resulting fibre mat was dried to remove any residual solvent, but not carbonized. The membranes were produced to have 33, 50, and $60 \mathrm{wt} \%$ PEI.

SEM images of the PS/PEI fibres showed a rough surface, which is ideal, increasing capacity for $\mathrm{CO}_{2}$ adsorption. The fibres also exhibited good $\mathrm{CO}_{2}$ selectivity, preferentially adsorbing $\mathrm{CO}_{2}$ over $\mathrm{N}_{2}$. Regeneration of PEI with adsorbed $\mathrm{CO}_{2}$ to its original state is a shortcoming for these fibres. $\mathrm{CO}_{2}$-breakthrough experiments were conducted with ambient air (400 ppm $\left.\mathrm{CO}_{2}\right)$ at various flow rates. It took 15 hours for $\mathrm{CO}_{2}$ to break through the $60 \mathrm{wt} \% \mathrm{PEI}$ membrane with air flowing at $30 \mathrm{ml} \cdot \mathrm{min}^{-1}$. The membrane was "regenerated" by heating it to $70{ }^{\circ} \mathrm{C}$ to drive off adsorbed $\mathrm{CO}_{2}$; in subsequent trials, the breakthrough time was reduced to 6 and 3 hours. The authors attribute this to incomplete desorption of $\mathrm{CO}_{2}$, as well as degradation of PEI at $70{ }^{\circ} \mathrm{C}$.

A zinc-air test cell was developed and battery performance did improve with the PEI membrane. An experiment was conducted without the membrane and a gas mixture of 5,000 ppm $\mathrm{CO}_{2}$ and air $\left(21 \% \mathrm{O}_{2}, 79 \% \mathrm{~N}_{2}\right)$ to confirm electrolyte degradation caused by $\mathrm{CO}_{2}$. A white powder formed on the air cathode and zinc foil during discharge; energy dispersive spectroscopy (EDS) analysis indicated that the substance was likely $\mathrm{K}_{2} \mathrm{CO}_{2}$, formed via reaction of $\mathrm{KOH}$ and $\mathrm{CO}_{2}$.

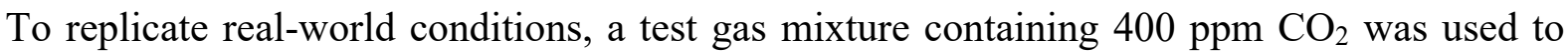
compare cell performance with a gas mixture close to that of atmospheric air. Addition of the PEI membrane increased discharge capacity from $762 \mathrm{~mA} \cdot \mathrm{h} \cdot \mathrm{g}^{-1}$ to $801 \mathrm{~mA} \cdot \mathrm{h} \cdot \mathrm{g}^{-1}$ at a current density of $10 \mathrm{~mA} \cdot \mathrm{cm}^{-2}$. The operating voltage of the PEI cell also increased slightly, from 1.24 $\mathrm{V}$ to $1.27 \mathrm{~V}$. Cycling tests with the air gas mixture did not show any benefit to including the PEI membrane, possibly due to the relatively low $\mathrm{CO}_{2}$ concentration.

This work showed that zinc-air batteries can benefit from the use of $\mathrm{CO}_{2}$ adsorbing membranes, though more development is needed for the benefit to be substantial. The cyclability of the adsorbent membrane needs improvement for longer duration performance. However, this is a novel application of electrospun materials to zinc-air batteries and one that represents an exciting possible future research direction.

Another application of electrospun materials to metal-air batteries is utilizing them as gas diffusion layer materials, in a similar manner to those used in fuel cells discussed in Section 
4.2. The high-density sheets of carbon fibres produced by electrospinning feature smaller fibres and a tighter pore structure than conventional carbon paper materials. Kim et al. have explored using electrospun carbon fibres as GDL materials, and have taken the additional step of texturing the surface of the spun carbon fibres to improve the transport of oxygen through the GDL [227].

The electrospinning process used to produce mats of carbon fibres is standard, a solution of PAN and DMF was spun and subsequently carbonized to produce high purity carbon fibres. Metal moulds were produced by pressing a wire template of the pattern with alternating current flowing through it into a metal substrate. A line pattern mould and a pyramid patterned mould were produced and they exhibited an increase in surface roughness compared to the flat collector of over $1,000 \%$. The pattern was transferred from the metal mould to the carbon fibre sheet by compressing the two together with at a pressure of $10 \mathrm{MPa}$ at $50{ }^{\circ} \mathrm{C}$.

The concept of producing carbon fibre materials with a surface roughness is to promote turbulent flow, increasing the velocity and vorticity of gases flowing through the GDL. The pyramid patterned design exhibited the best performance in a lithium-air battery cell. It had the lowest IR drop of the three materials tested, and a higher ORR current density and onset potential. The authors attribute these results to improved diffusion velocity of reactants to the cathode. Additionally, COMSOL modelling demonstrated that the pyramid patterned material had the smallest internal volume, and highest fluid velocity with the most vorticity of the materials studied. A model material, named Model-3, had void space filled with randomly placed cylindrical structures such that its internal volume was the lowest of all the cases studied; this material exhibited a higher velocity, attributed to the reduction in internal void space. To further explore the effects of a reduced internal volume in the GDL, pyramid patterned materials were produced using 5, 10, and $15 \mathrm{MPa}$ pressure. The $15 \mathrm{MPa}$ material did not outperform the $10 \mathrm{MPa}$ material, suggesting there is a limit to the amount that volume reduction can increase mass transport. This work illustrates the mass transport advantages that electrospun materials offer over conventional gas diffusion layers, an application of electrospun fibre sheets in metal-air cells that has yet to be fully explored.

\subsection{Conclusions and perspectives}

The facile synthesis of electrochemically active electrospun carbon fibres has resulted in a great deal of research for use as conventional PGM catalyst replacements. Not only do the carbon fibres act as supports for catalytic metal and metal oxide particles, carbon-nitrogen heteroatoms are also formed during their synthesis, further promoting ORR and OER reactions. The high 
specific surface area of composite fibres is a desirable result of metal particles formation during pyrolysis; the research reviewed here confirms that increasing the number of active sites available to catalyse oxygen reduction serves to increase the discharge capacity of metal-air cells. The role of metals and metal oxides in the fibres is complex, as they have demonstrated catalytic activity comparable to $\mathrm{Pt} / \mathrm{C}$ for the ORR reaction while also mediating the accumulation of discharge products, increasing discharge capacity and reducing charge potentials. The improved decomposition of discharge products, such as $\mathrm{Li}_{2} \mathrm{O}_{2}$, increases cycle stability of cathode materials, and some of the work discussed in this section has produced materials with greater stability than conventional Pt catalysts. Metal-free carbon fibres with nitrogen-doping have shown bifunctional catalyst capabilities and could serve to replace both $\mathrm{Pt} / \mathrm{C}$ and $\mathrm{Ir} / \mathrm{C}$ as ORR and OER catalysts, respectively. The electrospun fibres also show promise as a gas diffusion layer material, and the possibility of adding functionality to fibres in this component of the cell is beginning to be explored. Much of work with electrochemically active electrospun fibres involves grinding the fibre sheets and applying them as a catalyst layer, similar to how platinum on carbon would be handled to produce a catalyst layer. However, free-standing sheets of electrospun fibres are showing promise as catalyst layer materials, eliminating material processing steps and potentially offering a more elegant cell design.

\section{Supercapacitors}

Supercapacitors (SCs), also called ultracapacitors or electrochemical capacitors, hold an important place in the field of energy storage due to their ability to charge and discharge at very high rates (beyond tens of Amperes per gram of active material). Developed at the same time as Li-and Na-ion batteries in the 1970s [228,229], their very long cycle life (beyond 100,000 cycles, $\sim 15$ years) has always been attractive in applications where power pulses ( $>$ $10 \mathrm{~kW} \cdot \mathrm{kg}^{-1}$ ) are needed, for instance, to crank engines or recover braking energy. So far, their very limited energy density $\left(\sim 5 \mathrm{Wh} \cdot \mathrm{kg}^{-1}\right)$ compared to other battery technologies (i.e. 150 $200 \mathrm{Wh} \cdot \mathrm{kg}^{-1}$ for Li-ion, $60 \mathrm{Wh} \cdot \mathrm{kg}^{-1}$ for Ni-metal hydride) restrains their application as a power source for portable devices in a world where electrical device portability becomes the new norm. Thus, they are currently used to increase efficiency and cyclability of battery-powered e-vehicles via hybridisation [230]. However, with the recent progress in the design of electrically conductive nanostructures, supercapacitors are now able to power e-vehicles in urban areas due to low range requirements (i.e. buses, taxis, ferries that can frequently recharge en route). Their faster charging rates allows the recovery of breaking energy, bringing environmental benefits through better air quality [231-234]. 
The charge storage mechanism of supercapacitors relies on various reversible phenomena. The most widely commercialised and studied supercapacitor type is called the "electrical doublelayer capacitor" (EDLC). It relies on the surface electrosorption of electrolyte ions on a porous surface. The majority of these capacitive materials are high-surface-area porous carbon materials. The CVs of such supercapacitor devices are nearly rectangular and the galvanostatic charge/discharge (GCD) profiles triangular, indicating very fast non-Faradaic charge transfer. However, other, so-called pseudo-capacitive, materials such as reversible surface redox (e.g. $\mathrm{RuO}_{2}, \mathrm{MnO}_{2}, \mathrm{Ti}_{3} \mathrm{C}_{2} \mathrm{~T}_{x} \mathrm{MXenes}$ ) and intercalation compounds (e.g. $\mathrm{Nb}_{2} \mathrm{O}_{5}$ ) [235], comprise a growing proportion of the supercapacitor market and research. In contrast to battery-like materials, pseudo-capacitive materials exhibit redox processes which are not limited by bulk diffusion. Typically, the CV peaks are broader than diffusion-controlled peaks and the galvanostatic charge/discharge profiles exhibit rather elongated triangles. The distinction between the various charge storing mechanisms is crucial to improve the performances of supercapacitors. In the field of carbon materials, the presence of heteroatoms (e.g. N, P, S) embedded in the carbon framework can contribute significantly to the materials' performance via pseudo-capacitance [236]. Therefore, the new generation of carbon materials must not only exhibit a tuneable porosity but also controlled surface functional groups for improved performance. As it will be shown in this section, electrospinning represents a very promising synthesis method to prepare carbon and carbon-transition metal-hybrid materials for supercapacitors.

\subsection{Challenges for the next generation of supercapacitive carbon electrodes}

To improve marketability $\left(\$ \cdot \mathrm{Wh}^{-1}\right)$, the energy density of supercapacitors needs to be increased while keeping the costs low (Fig. 16a). As it is proportional to the electrode capacitance and the square of the cell voltage, the two main strategies to improve the energy density are to i) design porous materials with suitable pore size distributions and good electrolyte accessibility with better charge storage properties (capacitance increase) and ii) match the surface chemistry and pore size with high-stability electrolytes (cell voltage increase), employing organic solvents. Double-layer charge storage is maximised when the pore size approaches the unsolvated electrolyte ion size [237,238]. If this is achieved, the ions are forced by the electric field to enter the pores and lose part of their solvation shell. This desolvation mechanism reduces the ion-wall distance and increases the electrostatic interaction between the charged electrode and the ions and thus the capacitance. Thus, microporous $(0.5<d<1 \mathrm{~nm})$ materials 
with relatively low pore volumes $\left(<0.5 \mathrm{~cm}^{3} \cdot \mathrm{g}^{-1}\right)$ are highly desirable to maximise capacitance. To allow the electrolyte penetration into these micropores, the surface chemistry of the material must allow the best wettability possible with the chosen electrolyte [239]. For aqueous electrolytes, the wettability of carbonaceous surfaces improves with the presence of heteroatoms (e.g. oxygen, nitrogen, phosphorus, sulfur) [240]. In the case of organic electrolytes, carbon surfaces free from labile polar functional groups are required as they were shown to be unstable at high voltages (beyond $2.5 \mathrm{~V}$ ) [230]. In addition to a well-engineered carbon surface, the presence of a hierarchical porous structure comprising micro-, meso- and macropores facilitates the electrolyte diffusion into the micropores [241]. As a result of the last decade of research, graphene-based materials have emerged and shown great promise in highenergy-density devices $\left(>60 \mathrm{Wh} \cdot \mathrm{kg}^{-1}\right)$ due to their tuneable morphologies and surface functionalities [242]. Concurrently, the porosity development in activated carbons has become more efficient and a second generation of bio-based nanostructured carbons is becoming a sustainable alternative $[243,244]$.
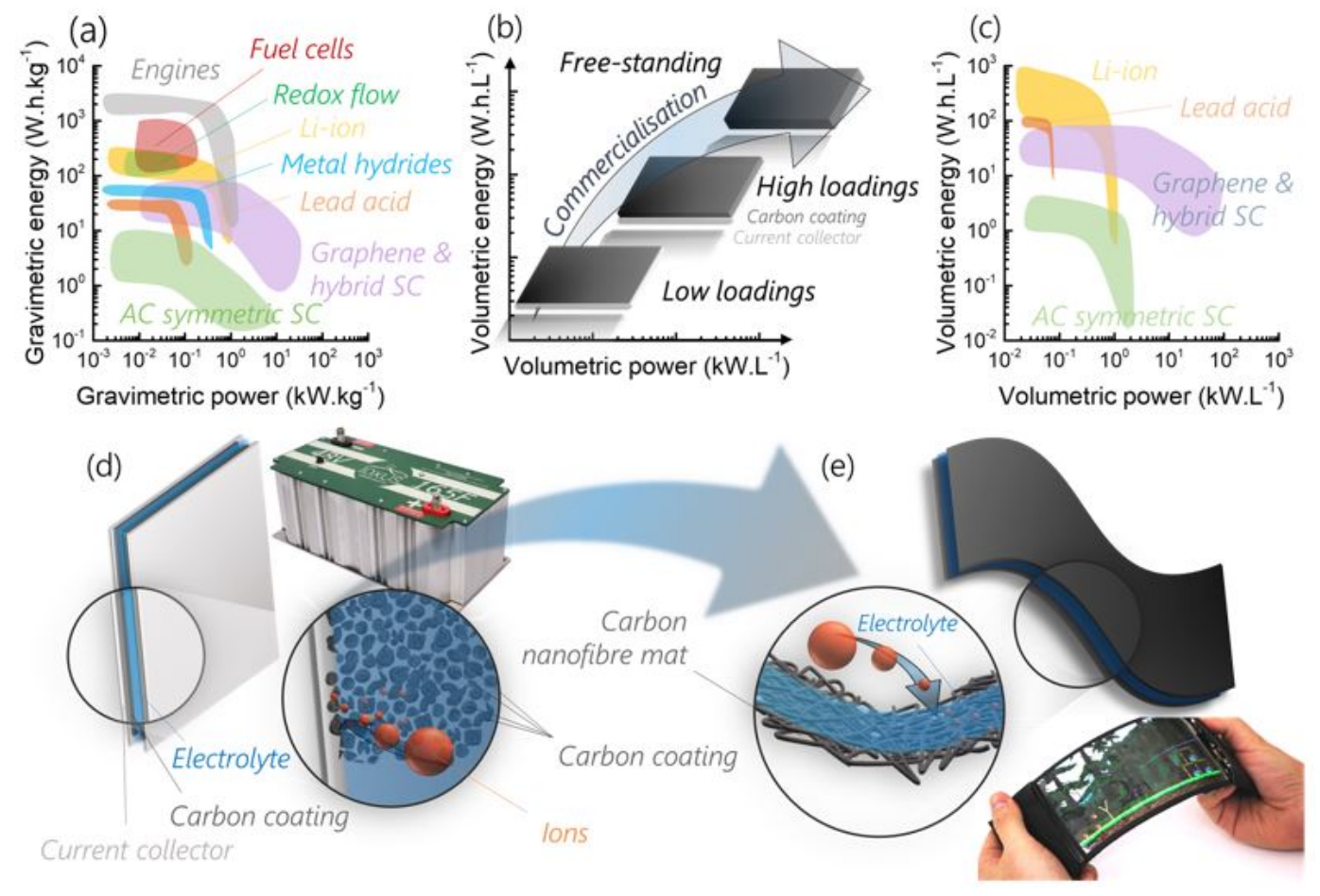

Fig. 16. (a) Gravimetric Ragone plot for various energy storage devices, (b) Ragone plot showing the variation of volumetric performances depending on the mass loading of active material, (c) volumetric Ragone plot for batteries and supercapacitors, (d) supercapacitive cell with film-coated carbon powder-based electrodes, (e) free-standing and flexible carbon nanofibre electrodes as electrodes in conjunction with a polymer electrolyte.

The vast majority of commercially available porous carbon materials are synthesised by steam activation of lignin-rich agricultural wastes, typically coconut shells, to maximise the carbon 
yield. However, the high metal content of these starting materials form carbon materials of low purity which catalyse the decomposition of the organic electrolyte and lead to performance loss. Thus, high purity carbons are required for good cyclability and the removal of these metal impurities, performed after activation, increases the synthesis costs to $15 \$ \cdot \mathrm{kg}^{-1}$ (in comparison to $10 \$ \cdot \mathrm{kg}^{-1}$ for commercially available graphite for Li-ion) [244]. Commercial electrodes in supercapacitors consist of a carbon film coated onto a metallic current collector [245]. Prior to coating, the surface of the current collector is etched and modified by a coating of conductive carbon to improve the adhesion of the active material [229]. The active material is mixed with a polymeric binder and a conductive additive (CNTs or carbon black) to form a slurry that can be coated with a controlled thickness, rolled, and dried to form a porous electrode (Fig. 16d). The thickness of the electrode (active layer + current collector) in commercial devices ranges from 100 to $300 \mu \mathrm{m}$ with a high porosity of $65-75 \%$, densities of 0.7 to more than $0.9 \mathrm{~g} \cdot \mathrm{cm}^{-3}$ and mass loadings of 8 to $10 \mathrm{mg} \cdot \mathrm{cm}^{-2}$ per electrode [229,245,246]. Therefore, to enter the market, newly developed carbon materials must exhibit densities higher than activated carbons $\left(\sim 0.5 \mathrm{~g} \cdot \mathrm{cm}^{-3}\right)$, be functional with commercial mass loadings and use low-cost resources with low ash contents (Fig. 16 (b and c)).

Electrospun carbon nanofibres (ECNFs) have been investigated extensively as new-generation electrodes for supercapacitors to replace the widely used powder-based, film-coated electrodes. Self-supported - also called free-standing - and flexible electrodes are sufficiently electrically conductive $\left(<1{\mathrm{~S} . \mathrm{cm}^{-1}}^{-1}\right)$ to not require additional metallic substrates. Thus, multiple process steps, such as current collector preparation, particle sizing and slurry coating, could be omitted through the nanofibre-based electrodes. In conjunction with a polymer electrolyte, the freestanding ECNF mats may also be suitable electrodes for the next-generation of energy storage devices that will be flexible and wearable (Fig. 16e) [247]. However, in order for electrospun carbon nanofibres to be implemented in real-life devices, two main obstacles remain to be overcome. Firstly, on a microscopic level, the low density of (activated) ECNFs prevents them from being used as an alternative to the film coated electrodes because the attained loading is very low $\left(1.0 \mathrm{mg} \cdot \mathrm{cm}^{-2}\right)$. Secondly, on the nanoscopic level, the reliable large scale and continuous thermal conversion of electrospun carbon nanofibres with defined pore sizes remains to be demonstrated. Despite these shortcomings, activated ECNFs have already shown

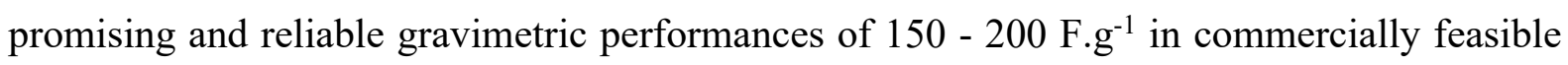
aqueous (i.e neutral $\mathrm{pH}$ ), organic and ionic liquids electrolytes and these recent results have been exhaustively summarized in the literature [248]. Thus, ECNFs already outperform 
consistently commercially available powdered carbons which usually exhibit capacitance values around $100 \mathrm{~F} . \mathrm{g}^{-1}$ in aqueous and $60 \mathrm{~F}^{-1} \mathrm{~g}^{-1}$ in organic electrolytes [249-251]. However, despite these results, understanding of the pore formation mechanisms is still lacking and systematic studies on how to reliably control the pore-size in the ion size range $(0.5-2 \mathrm{~nm})$ and surface functionalities are needed.

\subsection{Tuning carbon nanofibre properties for supercapacitive applications}

Tackling the low-density of the nanofibrous network can be achieved by several means. The charge density of the spinning solution can be increased, affecting the elongation of the jet and producing nanofibrous networks with larger packing density [252]. As it affects solely the micrometer-sized pores, the density can be doubled without affecting the supercapacitive performances. It is also possible to compress the electrospun mats to tune the density of the structure [253]. In this case the pressure must be carefully controlled to reduce the amount of micrometer-sized pores without transforming the fibres back into a non-porous film. Despite being promising, these two methods as yet have not been extensively reported and they should still be proven to be effective.

The large void volume between the fibres in ECNF serve as electrolyte reservoir, which makes the micropores on the fibre surfaces directly accessible. This considerably shortens the ionic path and facilitates the penetration of the electrolyte inside the porous network compared to other porous carbons with tortuous porous networks. However, the microporosity within the fibres can still be tortuous and the effect of surface chemistry increasingly impacts the wettability as the pore size becomes smaller. As mentioned in the previous sections, microporosity in EDLC supercapacitive materials is often produced by chemical or physical activation. For chemical alkali metals activation, microporosity can be tuned by changing the cation of the activating agent. Using carbon xerogels as standard model carbon, it was shown that moving from $\mathrm{Na}^{+}, \mathrm{K}^{+}, \mathrm{Rb}^{+}$and $\mathrm{Cs}^{+}$, the pores size could be tuned from 0.6 to $0.8 \mathrm{~nm}$. The charge storage performances remain, however, to be tested [254]. Controlling precisely the size of the micropores using physical activation (i.e $\mathrm{H}_{2} \mathrm{O}$ or $\mathrm{CO}_{2}$ ) is still challenging. Other bottomup methods such as the synthesis of block-co-polymers such as PAN-b-PMMA could be shown to be promising but they need to be further investigated [255].

The mechanical properties and microporosity of ECNF are tightly linked by the crystallisation degree of the material [256]. As a general rule, increasing the pyrolysis temperature promotes 
the formations of larger aromatic domains which stack into crystallites. This tends to close the micropores and reduce the overall porosity while increasing conductivity and mechanical properties. The crystallisation degree can also be tuned by using thermally stable precursors with various chemical structures. Amorphous polymers (i.e lignin, cellulose) will form nongraphitisable hard carbons at high temperatures whereas semi-crystalline polymers such as PAN form large graphitic domains. The kinetics of crystallisation can also be tuned by applying sufficient pressure on the materials during thermal treatment as it reduces the mean interatomic distance [257]. It is also possible to tune the crystallinity by applying stress on aligned fibre mats during thermal treatment $[258,259]$ or by aligning the polymer chains during the spinning, either by addition of unidirectional templates (i.e CNTs) or by elongating the jet using a rotating drum collector [260]. Finally, this relationship between crystallinity and microporosity shall be further understood in order to choose the adequate thermal treatment to each precursor. This would allow to tune nanostructures and maximise performances.

\subsection{Promising electrospun carbon nanofibrous architectures based mostly on EDLC capacitance}

One of the most widely used electrospinning solution systems to produce carbon nanofibres as supercapacitor electrodes is based on PAN dissolved in DMF, which is also widely used in electrospinning for RFB, fuel cell and metal-air battery applications, as already outlined. PAN is a chemically well-defined and structurally ordered synthetic polymer which is linear and rich in nitrogen (polar nitrile groups) [261]. Hence, the creation of microporous carbons is only achievable under harsh conditions with HCN as a toxic by-product [262]. However, the high content of nitrogen in the precursor yields pseudo-capacitive nitrogen functionalized ECNFs, which enable high capacitance values in aqueous electrolytes. Nitrogen functional groups provide a lone electron pair to the carbon network. This has the effect of modifying the bandgap of the overall material and increasing the capacitive ion sorption [263]. The pseudocapacitive signature of nitrogen functional groups is also often observed as a broad peak on a CV because the charge redistribution on the carbon skeletons makes the active sites merge into the same energy state $[240,263]$.

Electrospun PAN nanofibres were introduced by Kim et al. in 2003 who activated the precursor fibres by carbonisation in a mixture of $30 \mathrm{vol} \%$ steam and $70 \mathrm{vol} \% \mathrm{~N}_{2}$ for $30 \mathrm{~min}$ [264]. The resulting ECNF electrodes showed a maximum gravimetric capacitance of $173 \mathrm{~F} \cdot \mathrm{g}^{-1}$ at 
$0.01 \mathrm{~A} \cdot \mathrm{g}^{-1}$ and $120 \mathrm{~F} \cdot \mathrm{g}^{-1}$ at $1 \mathrm{~A} \cdot \mathrm{g}^{-1}$ in an aqueous alkaline electrolyte $(6 \mathrm{M} \mathrm{KOH})$. Ra et al. modified the activation procedure of PAN-ECNFs [265]. They activated PAN-ECNFs with $\mathrm{CO}_{2}$ at $700-1,000{ }^{\circ} \mathrm{C}$ for four hours. These ECNF electrodes were tested in aqueous $1 \mathrm{M}$ $\mathrm{H}_{2} \mathrm{SO}_{4}$ and $6 \mathrm{M} \mathrm{KOH}$, and in $1 \mathrm{M}$ tetraethylammonium tetrafluoroborate dissolved in acetonitrile. It was shown that an activation temperature of $900{ }^{\circ} \mathrm{C}$ yielded the best results in aqueous electrolytes exceeding $200 \mathrm{~F} \cdot \mathrm{g}^{-1}$ at low current densities $\left(<0.1 \mathrm{~A} \cdot \mathrm{g}^{-1}\right)$ and retaining more than $150 \mathrm{~F}^{-1} \mathrm{~g}^{-1}$ at a high current density of $50 \mathrm{~A} \cdot \mathrm{g}^{-1}$ [265]. This was assigned to the development of (sub-)nanometre pores in combination with the high abundance of nitrogen surface functional groups (6.9 wt \%), which give rise to pseudocapacitive contributions [265].

In organic electrolytes, the highest capacitance values of about $100 \mathrm{~F} \cdot \mathrm{g}^{-1}$ at low current densities and still more than $70 \mathrm{~F} \cdot \mathrm{g}^{-1}$ at a high current density of $50 \mathrm{~A} \cdot \mathrm{g}^{-1}$ were attained by activation at $1,000{ }^{\circ} \mathrm{C}$ [265]. This was attributed to the well-developed pore size distribution with greater pore diameters (micro- and mesopores) accommodating the larger ions of the organic electrolyte. However, under these harsh activation conditions, only a minor amount of nitrogen functional groups remained $(3.3 \mathrm{wt} \%)$ on the ECNF surface. Moreover, several activating agents were incorporated into the PAN-DMF electrospinning solution to increase the specific surface area. However, the resulting values of the capacitance and the energy and power densities do not exceed the results of physical activation by steam or $\mathrm{CO}_{2}$ [266-268]. Conversely, sacrificial templating with polymethyl methacrylate (PMMA) together with metal (Ni) doping has shown an improved rate capability compared to solely activated PAN-ECNFs [269].

Another type of precursor that is widely used in the preparation of ECNFs as supercapacitor electrodes are lignins [262,270-272]. Contrary to PAN, lignins are nature-made, oxygenated and amorphous biopolymers synthesized in a non-linear fashion via combinatorial oxidative coupling reactions in the cell wall of vascular plants (e.g. trees) [273]. This gives rise to biopolymers with a highly crosslinked and amorphous, three-dimensional structure. Thus, carbonisation without any additional activation step was shown to yield highly microporous and oxygen functionalized ECNFs [274]. This class of renewable polymer precursors is chemically highly variable depending on the plant source, extraction method and refinement procedure $[152,275]$. This gives rise to a vast array of different lignins with various chemical compositions and structural features [276]. Hence, revealing structure-property relationships 
in the field of electrospun lignin-derived carbon nanofibres (LCNFs) is complex and often highly dependent on the source of lignin used in the work [274].

The first reports of lignin-derived carbon nanofibres from lignin extracted with organic solvents (Organosolv process) via co-axial electrospinning were published by Lallave et al. in 2007 [277], Berenguer et al. showed that microporosity develops intrinsically during carbonisation of lignin nanofibres, without any additional activation process which is related to the high oxygen content in lignins (up to $30 \mathrm{wt} \%$ ) [278]. Furthermore, they showed that the porous LCNFs incorporate redox-active quinone-like functional groups which resulted in a high capacitance of $188 \mathrm{~F} \cdot \mathrm{g}^{-1}$ at a low current density of $1 \mathrm{~A} \cdot \mathrm{g}^{-1}$, dropping to $120 \mathrm{~F} \cdot \mathrm{g}^{-1}$ at high current densities of up to $100 \mathrm{~A} \cdot \mathrm{g}^{-1}$ in $1 \mathrm{M} \mathrm{H}_{2} \mathrm{SO}_{4}$ electrolyte [278]. The importance of the high oxygen content in the carbonised material for the capacitive performance as supercapacitor electrodes was highlighted more recently [151]. Therein, it was shown that highly porous, oxygen functionalized LCNFs can be efficiently produced by electrospinning lignin dissolved in $\mathrm{NaOH}_{(\mathrm{aq})}$. Hence, forty times less activating agent was required compared to traditionally chemically activated carbons [151]. Furthermore, it has been described that the rapid evaporation of the aqueous alkaline solution during electrospinning promotes the development of porosity by increasing the interfacial surface between the activating agent and the carbon framework. This optimises the activation step by reducing the amount of activating agent needed to form high surface area materials [151,279]. A scheme of the CNF properties from PAN and lignins are provided in Fig. 17.

Polymer precursor

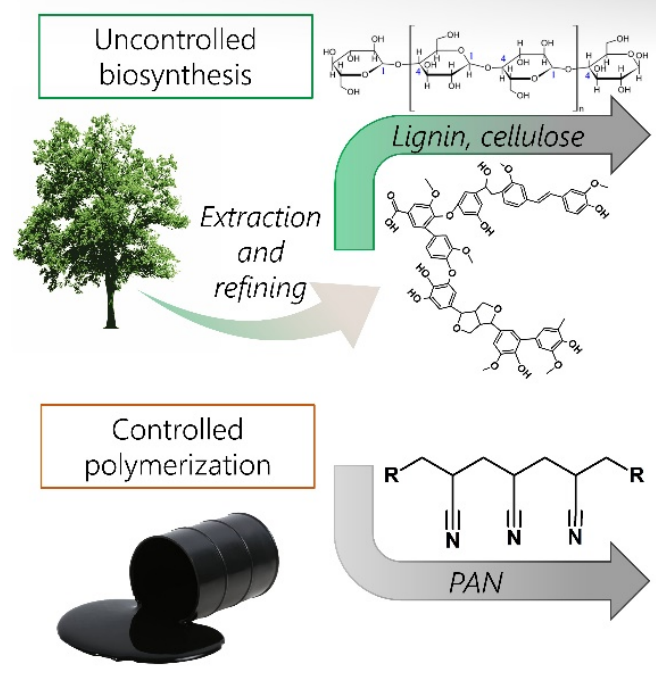

ECNF properties

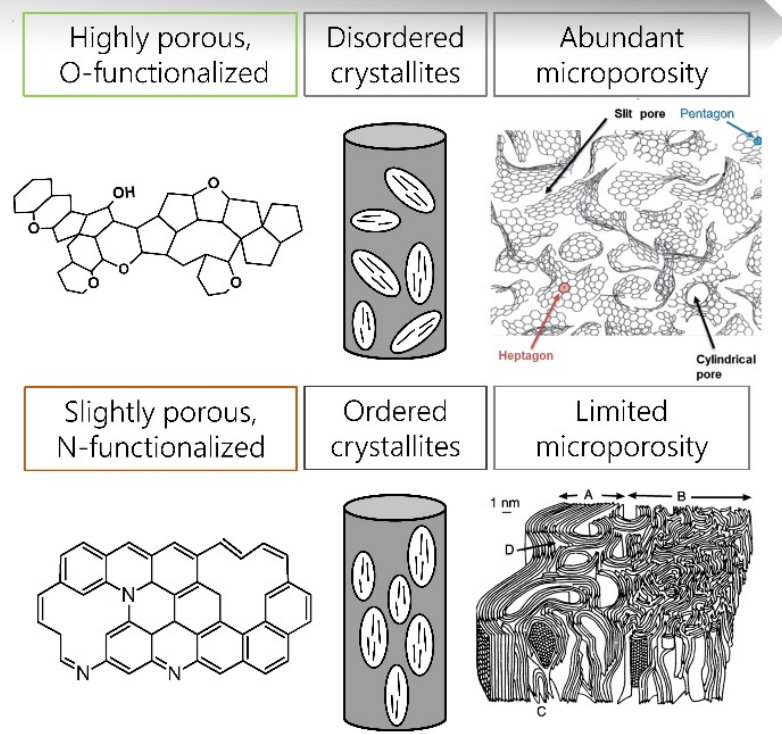


Fig. 17. Relationship between precursor properties (left) and ECNF properties (right) for biomass-derived polymers and synthetic polymers such as PAN. Reproduced from Ref. [259,280] with permissions from John Wiley and Sons.

To combine the merits of $\mathrm{N}$-functionalization and a high degree of microporosity solely based on renewable precursors, Yang et al. blended lignin with the plant proteins (hordein/zein) achieving a maximum nitrogen content of only 1.9 at $\%$ with a moderately high specific surface area of $560 \mathrm{~m}^{2} \cdot \mathrm{g}^{-1}$ [281]. However, it was not the sample with the highest nitrogen (protein) content that showed the highest capacitance in $6 \mathrm{M} \mathrm{KOH}_{(\mathrm{aq})}$ electrolyte, but the sample with the highest specific surface area (highest lignin content). This shows that blending two polymers with complementary chemical compositions might not be the most effective route towards well-engineered functional ECNFs for supercapacitors.

Besides the use of ECNFs as the sole active electrode material, their use as electrically conductive scaffolds to produce transition metal oxide/hydroxide-carbon composite electrodes for supercapacitors is a promising strategy to increase the energy density. This approach increases not only the specific gravimetric $\left(\mathrm{Wh}_{\mathrm{kg}}{ }^{-1}\right)$, but also the specific volumetric energy $\left(\mathrm{Wh} \cdot \mathrm{m}^{-3}\right)$ density of the ECNF electrodes as metal oxides are much more dense than carbon materials. In particular, the low volumetric energy density of ECNF electrodes is a technological bottleneck for the industrial adoption of this new kind of electrode materials.

\subsection{Promising electrospun carbon nanofibrous architectures based mostly on pseudo-capacitance}

As previously mentioned, transition metal oxide materials possess pseudocapacitive properties. In electrically conducting materials and in materials with short separation between the active sites, the redox-active sites can interact with each other and form a broad energy band [240]. In conducting materials (e.g. metals and conducting polymers), this band corresponds to the delocalised electronic network. When the electrode is positively polarised and the overpotential is sufficient, the electrons from the ions are transferred to the material and merge into the broad band. The ion will adsorb on the surface, but the electron transferred by the Faradaic process will be directly transferred to the charging device. No charge is stored inside the conductor and the surface electron density only depends on the electrode polarisation coming from the electric field. In semiconductor materials with bandgaps, like the transition metal oxides, a slight overpotential of several hundreds of millivolts can in certain cases cause the electron to be promoted from the valence band to the conduction band. The overpotential set by the charging device leads to a polarisation of the material; this polarisation, depending on the permittivity of the material, can be extremely weak considering that the observation of redox reactions in 
the liquid phase is on the order of several volts. This polarisation will create electrostatic interactions on the surface of the material and a charge transfer might occur.

$\mathrm{MnO}_{2}$ is considered to be one of the most promising electrode materials in supercapacitors due to its high abundance, low price and low toxicity [282,283]. The main drawbacks of $\mathrm{MnO}_{2}$ are its low electric conductivity and its instability during repeated charging and discharging due to dissolution which results in low durability. Combining $\mathrm{MnO}_{2}$ with ECNFs has been shown to be a viable route towards improved electric conductivity and stability upon cycling [284]. Ma et al. manufactured flexible LCNF mats by electrospinning aqueous solutions of Kraft lignin and polyvinyl alcohol (PVA) in order to use them as conductive substrates for $\mathrm{MnO}_{2}$ (Fig. 18a) [285]. After stabilization and carbonisation, $\mathrm{MnO}_{2}$ was deposited in a separate step by immersing a LCNF mat into aqueous $\mathrm{KMnO}_{4}$ solutions at $65{ }^{\circ} \mathrm{C}$ for four hours. The resulting composite electrodes were then tested in an organic electrolyte [285]. The maximum performance of $83.3 \mathrm{~F} \cdot \mathrm{g}^{-1}, 84.3 \mathrm{Wh} \cdot \mathrm{kg}^{-1}$, and the maximum power density of $5.72 \mathrm{~kW} \cdot \mathrm{kg}^{-1}$ were achieved by the composite electrode with an equal mass ratio of $\mathrm{MnO}_{2}$ to carbon. After 10,000 cycles, the specific gravimetric capacitance value of the $\mathrm{MnO}_{2}-\mathrm{ECNF}$ composite decreased only by approximately $8 \%$, which is promising. However, the volumetric energy and power density were not reported in this study.

(a)

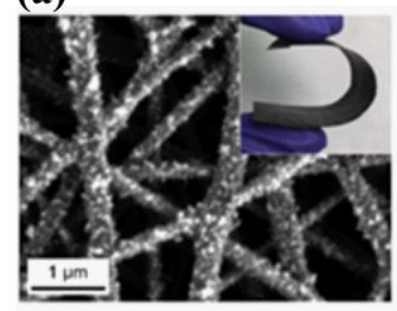

(c)

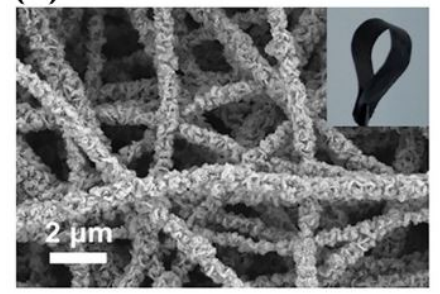

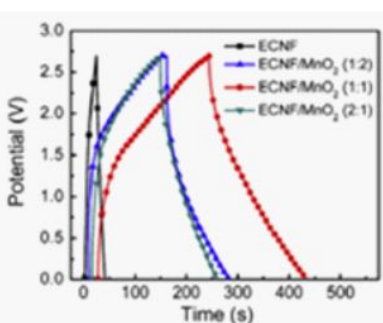

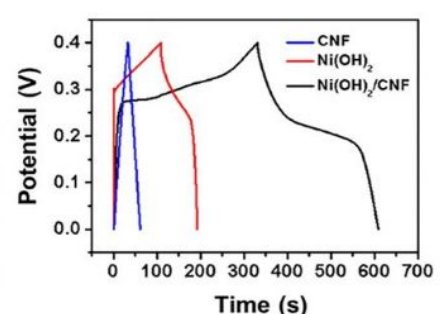

(b)

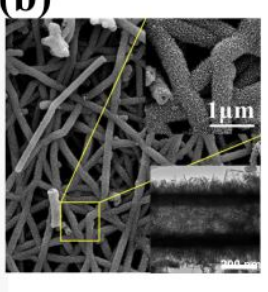

(d)

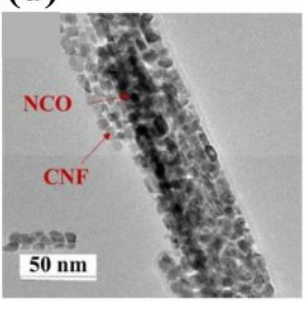

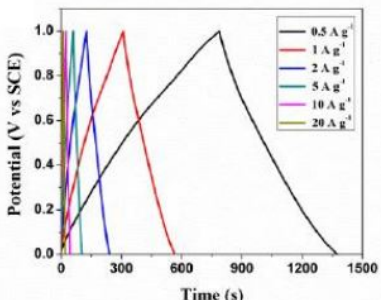

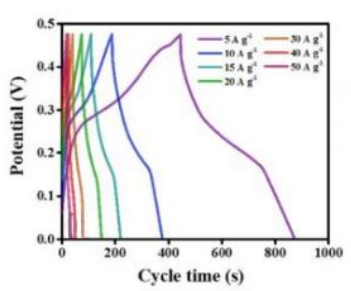

Fig. 18. Use of electrospun carbon nanofibres as electrically conductive scaffolds for the deposition of $\mathrm{MnO}_{2}(\mathrm{a}$ and $\mathrm{b})$, $\mathrm{Ni}(\mathrm{OH})_{2}(\mathrm{c})$ and $\mathrm{NiCO}_{2} \mathrm{O}_{4}$ (d) particles [285-288]. Reproduced from Ref [286] with permission from American Chemical Society.

Zhao et al. fabricated hybrid hollow ECNFs which were decorated with $\mathrm{MnO}_{2}$ nanosheets (Fig. 18b) [288]. The hollow structure was created by co-electrospinning PAN and PVP solutions followed by carbonisation at $800{ }^{\circ} \mathrm{C}$ under nitrogen. The formation of the hollow structure was 
due to immiscibility between PAN and PVP. The authors showed that the hollow hybrid ECNFs exhibited better capacitive performance $\left(293.6 \mathrm{~F} \cdot \mathrm{g}^{-1}\right.$ at $0.5 \mathrm{~A} \cdot \mathrm{g}^{-1}$ in $1 \mathrm{M} \mathrm{Na}_{2} \mathrm{SO}_{4}$ electrolyte) compared to non-hollow fibres which was attributed to improved accessibility and better interfacial contact between the carbon nanofibres and the $\mathrm{MnO}_{2}$ nanosheets. Unfortunately, it is still challenging to reach similar performances as the $\mathrm{MnO}_{2}$ structure grown on other thin conductive substrates where the growth is much more controlled than on carbon substrates [289].

$\mathrm{Ni}(\mathrm{OH})_{2}$ and $\mathrm{NiO}$ are also promising redox-active compounds as they are abundant and hence low-cost materials. However, $\mathrm{Ni}^{2+}$ is classified as carcinogenic [290], which is a concern if an electrochemical cell fails and the cell interior gets exposed to air. Additionally, both compounds suffer from low electrical conductivity and instability during cycling due to large volume expansion [286]. In order to overcome the latter problems and take advantage of the redox-activity of $\mathrm{Ni}(\mathrm{OH})_{2}$, Zhang et al. grew $\mathrm{Ni}(\mathrm{OH})_{2}$ nanoplatelets vertically on ECNFs (Fig. 18c) [286]. They deposited $\mathrm{Ni}(\mathrm{OH})_{2}$ on the pre-fabricated ECNF substrate via urea assisted precipitation reaction [286]. The ECNF networks were reported to facilitate the charge transfer across the electrode-electrolyte-interface and mitigating the large volumetric expansion of $\mathrm{Ni}(\mathrm{OH})_{2}$ upon cycling and hence improving the long-term stability [286]. However, the composite electrode was only tested in a three-electrode set-up with a platinum counter electrode and hence the electrochemical performance is not representative for an actual twoelectrode cell. Luan et al. deposited $\mathrm{NiO}$ nanoflakes on a carbon cloth substrate by a seedmediated hydrothermal method [291]. By combining the NiO-ECNF electrode with a reduced graphene electrode a supercapacitor was fabricated that could operate at a voltage of $1.7 \mathrm{~V}$ in $1 \mathrm{M} \mathrm{KOH}_{(\mathrm{aq})}$. The device achieved a high areal capacitance of $248 \mathrm{mF} \cdot \mathrm{cm}^{-2}$ and a high gravimetric energy density of $39.9 \mathrm{Wh} \mathrm{kg}^{-1}$ with a high mass loading of $5 \mathrm{mg}$ [291].Tomboc et al. prepared hollow ECNFs using co-axial electrospinning method, with PVP in the core and PAN in the shell (Fig. 18d) [287]. After electrospinning, PVP was leached out by soaking the as-spun composite in water to obtain a hollow structure [287]. This is a more efficient and benign strategy to remove the template than the removal by thermal treatment as it is possible to recover the template from the aqueous solution in an additional step. The hollow ECNFs were ground and then dispersed in the precursor solution for the active material $\left(\mathrm{NiCO}_{2} \mathrm{O}_{4}\right)$. This dispersion was then treated hydrothermally to create the final $\mathrm{NiCo}_{2} \mathrm{O}_{4}-\mathrm{ECNF}$ composite material. The hollow ECNFs provided a conductive network that enabled the movement of electrons from the active material to the current collector and electrolyte to the active material. 
However, the authors were not able to fabricate free-standing electrodes from these composite ECNFs.

Finally, ECNFs have also been shown to improve the supercapacitive performances of other promising materials such as $\mathrm{Nb}_{2} \mathrm{O}_{5}$ and $\mathrm{MXenes} \mathrm{have} \mathrm{emerged} \mathrm{quite} \mathrm{recently} \mathrm{as} \mathrm{highly} \mathrm{suitable}$ candidates as supercapacitor electrode materials. However, the synthesis of MXenes still requires harsh acidic conditions to open up the layered structures (i.e hydrofluoric acid), which clearly hinders their large scale applicability, unless other acids are found as replacement (i.e $\mathrm{HCl}$ ) [292]. In order to make them available to the class of flexible electrodes, MXenes have been incorporated into PAN-ECNFs [293]. Levitt et al. showed that for this material it is preferable to incorporate the active material into the ECNFs rather than depositing it on the outside to improve durability and rate capability of the composite material [293]. In the case of $\mathrm{Nb}_{2} \mathrm{O}_{5}$, the ECNF allowed better stability of the material by creating electronically conductive pathways between the pseudo-capacitive $\mathrm{Nb}_{2} \mathrm{O}_{5}$ [294]. An inherent drawback with incorporating active materials into electrospun fibres is the limited achievable loading of active material in the fibrous material due to the high void volume. This is a common problem in electrospinning which arises from electrostatic repulsion on the collector due to charge accumulation $[295,296]$. This, however, may be mitigated by applying a progressively more negative potential to the collector in the course of the electrospinning process.

\subsection{Outlook}

Overall, ECNFs can be fabricated starting from various polymer precursors yielding different pore structures, surface chemistries and degrees of structural order. The flexibility of the electrospinning process makes it possible to manufacture composite electrodes with various fibre morphologies and chemical compositions, which show promising results by increasing the gravimetric energy density. However, data on volumetric energy densities and areal capacitance of electrodes are scarce for electrospun supercapacitor electrodes. This is a major gap in the literature, which needs to be filled. Finally, due to its versatility, low-cost and scalability, electrospinning will be the key enabling technology for the manufacture of flexible electrodes required for flexible and even wearable supercapacitors in the future.

\section{Overall summary and outlook}

In this review, the application of electrospinning has been summarised for four key electrochemical energy devices; RFBs, fuel cells, metal air batteries and supercapacitors. In 
RFBs, the use of electrospun materials was initially as a 'catalyst layer' to provide a high surface area additive to the commercial carbon fibre electrodes, before exploration of freestanding electrospun electrodes aimed to entirely replace the commercial electrode structures. However, these early attempts suffered from poor mass transport properties, hindering their performance, particularly at high current densities or low states of charge. The research that has produced the best results in recent years has combined the advantages of small fibres from electrospinning with innovative ways to increase the porosity and permeability of the electrodes. Finally, use of alternative polymers to PAN is showing some early promise for increasing the surface functionality, and use of catalyst precursors in the electrospinning solution can produce nanoparticles to catalyse RFB redox processes. Future work in this area should always include some aspect of full cell testing as the microstructure of ECNFs can have a large bearing on the mass transport properties of the electrodes, which is missed when testing with CV only. In addition, where possible, a combination of 3D characterisation (using e.g. Xray $\mathrm{CT}$ ) and image-based modelling should be applied to fully understand the structureperformance relationship for RFBs.

Electrospinning is a highly versatile and tuneable technique that can produce high surface area carbon fibres for energy storage and conversion devices with controlled morphology. In many electrochemical devices, the structure-performance relationship is key to efficient operation, and as such ECNFs show great promise as a route to the optimisation of the microstructure for these applications - particularly where novel adaptations of the basic electrospinning process lead to materials with higher permeability than conventional ECNF mats, or a degree of alignment that promotes effective mass transport in a particular direction and improved electrical conductivity. In this review, the historical development and recent progress of electrospinning in RFBs, fuel cells, metal-air batteries and supercapacitors has been presented, with a wide range of electrospinning techniques leading to novel materials that show great promise in these electrochemical devices.

In addition to the controlled microstructure, electrospinning allows a variety of polymers or metal precursors to be employed, leading to materials with catalytic activity for a range of redox reactions through doping of the carbon structure or embedded catalyst nanoparticles. Studies that apply these catalytic enhancements in tandem with optimised microstructure for mass transport (and with electrochemical testing that takes into account the mass transport effects), characterised with advanced X-ray imaging techniques and computational modelling, 
have produced the best results in recent years. It is recommended that future studies focus on both the activity and mass transport properties of electrospun materials, conducting testing in full cell devices where applicable to fully demonstrate their commercial potential, noting that static methods such as CV do not tell the whole picture. For this potential to be fully realised, scalable electrospinning using methods such as multiple spinnerets must be achieved to translate lab-scale progress to commercially relevant production rates.

Finally, the petrochemically-derived polymers often used in electrospinning, particularly the most prevalent PAN, are beginning to be replaced by biomass-derived sustainable alternatives such as lignin. This not only has an environmental impact, but can also lead to increased functionality or microporosity of the fibre surfaces, improving performance in working electrochemical devices. Initial progress in this area is encouraging, with future work predicted to demonstrate that the materials employed in electrochemical devices of the future will themselves be sustainably sourced, leading to a fully green solution to the electrification of the transport and grid-scale storage sectors.

\section{Acknowledgements}

Y.W acknowledges the Chinese Scholarship Council (CSC)/University College London for joint PhD scholarship, J.P.V.T to CONACyT for funding of his PhD scholarship, M-MT and PS thank RISE AB for co-funding Philipp Schlee's PhD position, P.R.S and M-M.T. acknowledge the support of the Royal Academy of Engineering, R.J, P.R.S, D.J.L.B and MM.T acknowledge the Faraday Institution for funding of electrochemical research in the EIL and Imperial under EP/S003053/1 


\begin{tabular}{|c|c|}
\hline \multicolumn{2}{|r|}{ Abbreviation list } \\
\hline Abbreviation & Definition \\
\hline AEAPT & [3-(2-aminoethylamino)propyl]trimethoxysilane \\
\hline ADT & accelerated degradation tests \\
\hline $\mathrm{AFC}$ & alkaline fuel cell \\
\hline $\mathrm{AC}$ & alternating current \\
\hline BET & Brunauer-Emmett-Teller \\
\hline $\mathrm{CF}$ & carbon felt \\
\hline $\mathrm{CT}$ & computed tomography \\
\hline $\mathrm{CV}$ & cyclic voltammogram \\
\hline $\mathrm{DMF}$ & dimethyl formamide \\
\hline $\mathrm{DC}$ & direct current \\
\hline eGDLs & electrospun gas diffusion layers \\
\hline ECSA & electrochemical surface area \\
\hline ECNF & electrospun carbon nanofiber \\
\hline EDLC & electric double layer capacitor \\
\hline EDS & energy dispersive spectroscopy \\
\hline $\mathrm{EE}$ & energy efficiency \\
\hline EIS & electrochemical impedance spectroscopy \\
\hline eMPL & electrospun equivalent microporous layer \\
\hline EOL & ethanol organosolv lignin \\
\hline FIB & focused ion beam \\
\hline GCD & galvanostatic charge/discharge \\
\hline GDL/GDL & gas diffusion layer \\
\hline HRSEM & high resolution scanning electron microscopy \\
\hline HRTEM & high resolution transmission electron microscopy \\
\hline HER & hydrogen evolution reaction \\
\hline $\mathrm{KL}$ & kraft lignin \\
\hline LBM & lattice Boltzmann method \\
\hline LCNF & lignin-derived carbon nanofibre \\
\hline LSV & linear sweep voltammetry \\
\hline LRSEM & low resolution scanning electron microscopy \\
\hline LRTEM & low resolution transmission electron microscopy \\
\hline MEA & membrane electrode assembly \\
\hline MIP & mercury intrusion porosimetry \\
\hline MPL & microporous layer \\
\hline OER & oxygen evolution reaction \\
\hline ORR & oxygen reduction reaction \\
\hline PL & phosphoric acid lignin \\
\hline PGM & platinum group metal \\
\hline PAN & polyacrylonitrile \\
\hline PEI & polyethyleneimine \\
\hline PI & polyimide \\
\hline PMMA & polymethyl methacrylate \\
\hline PS & polystyrene \\
\hline PVA & polyvinyl alcohol \\
\hline
\end{tabular}




\begin{tabular}{|c|c|}
\hline $\begin{array}{l}\text { PVP } \\
\text { PEMFC } \\
\text { PEMWE } \\
\text { RFB } \\
\text { RGO } \\
\text { SC } \\
\text { SEM } \\
\text { STEM } \\
\text { SoC } \\
\text { TEM } \\
\text { VRFB } \\
\text { XPS } \\
\text { XRD }\end{array}$ & $\begin{array}{l}\text { polyvinylpyrrolidone } \\
\text { proton exchange membrane fuel cell } \\
\text { proton exchange membrane water electrolyser } \\
\text { redox flow battery } \\
\text { reduced graphene oxide } \\
\text { Supercapacitor } \\
\text { scanning electron spectroscopy } \\
\text { scanning transmission electron microscopy } \\
\text { states of charge } \\
\text { transmission electron spectroscopy } \\
\text { vanadium redox flow battery } \\
\text { X-ray photoelectron spectroscopy } \\
\text { X-ray powder diffraction }\end{array}$ \\
\hline
\end{tabular}




\section{References}

[1] J.F. Cooley, US Pat. 692,631 693 (1902) 1-6, http://doi.org/10.1016/j.joen.2014.07.033.

[2] B.Y. Sir, G. Taylor, (1964).

[3] R.S. Barhate, S. Ramakrishna, J. Memb. Sci. 296 (2007) 1-8, http://doi.org/10.1016/j.memsci.2007.03.038.

[4] S.S. Ray, S.S. Chen, C.W. Li, N.C. Nguyen, H.T. Nguyen, RSC Adv. 6 (2016) 8549585514, http://doi.org/10.1039/c6ra14952a.

[5] M. Mirjalili, S. Zohoori, J. Nanostructure Chem. 6 (2016) 207-213, http://doi.org/10.1007/s40097-016-0189-y.

[6] Z. Liu, S. Ramakrishna, X. Liu, APL Bioeng. 4 (2020) 030901, http://doi.org/10.1063/5.0012309.

[7] A. Keirouz, M. Chung, J. Kwon, G. Fortunato, N. Radacsi, Wiley Interdiscip. Rev. Nanomedicine Nanobiotechnology 12 (2020) 1-32, http://doi.org/10.1002/wnan.1626.

[8] S. Lakshmi I, M. Varma K, G.S. Sajjan, K. Satish R, S. D, S. M, Int. J. Dent. Mater. 02 (2020) 37-44, http://doi.org/10.37983/ijdm.2020.2202.

[9] B. Pant, M. Park, S.J. Park, Pharmaceutics 11 (2019), http://doi.org/10.3390/pharmaceutics11070305.

[10] M.E. Orazem, Curr. Opin. Electrochem. 20 (2020) A2-A4, http://doi.org/10.1016/j.coelec.2020.06.008.

[11] J.G. López-Covarrubias, L. Soto-Muñoz, A.L. Iglesias, L.J. Villarreal-Gómez, Materials (Basel). 12 (2019) 1-18, http://doi.org/10.3390/ma12193190.

[12] J. Parbey, Q. Wang, G. Yu, X. Zhang, T. Li, M. Andersson, Rev. Chem. Eng. 0 (2019), http://doi.org/10.1515/revce-2018-0074.

[13] J.W. Jung, C.L. Lee, S. Yu, I.D. Kim, J. Mater. Chem. A 4 (2016) 703-750, http://doi.org/10.1039/c5ta06844d. 
[14] P. Robert Ilango, S. Peng, Curr. Opin. Electrochem. 18 (2019) 106-112, http://doi.org/10.1016/j.coelec.2019.10.016.

[15] S. Santangelo, Electrospun Nanomaterials for Energy Applications: Recent Advances, 2019, http://doi.org/10.3390/app9061049.

[16] M. Liu, N. Deng, J. Ju, L. Fan, L. Wang, Z. Li, H. Zhao, G. Yang, W. Kang, J. Yan, B. Cheng, Adv. Funct. Mater. 29 (2019) 1-34, http://doi.org/10.1002/adfm.201905467.

[17] Y. Wang, Y. Liu, Y. Liu, Q. Shen, C. Chen, F. Qiu, P. Li, L. Jiao, X. Qu, J. Energy Chem. 54 (2021) 225-241, http://doi.org/10.1016/j.jechem.2020.05.065.

[18] E.S. Cozza, O. Monticelli, E. Marsano, Macromol. Mater. Eng. 295 (2010) 791-795, http://doi.org/10.1002/mame.201000214.

[19] V. Mottaghitalab, M. Farjad, J. Polym. Eng. 33 (2013) 857-873, http://doi.org/10.1515/polyeng-2013-0081.

[20] Z. Cao, C. Wang, J. Chen, Mater. Lett. 225 (2018) 157-160, http://doi.org/10.1016/j.matlet.2018.05.007.

[21] C. Chen, S. Liu, W. Liu, Y. Zhao, Y. Lu, Sol. Energy Mater. Sol. Cells 96 (2012) $202-$ 209, http://doi.org/10.1016/j.solmat.2011.09.057.

[22] M. Gensheimer, M. Becker, A. Brandis-Heep, J.H. Wendorff, R.K. Thauer, A. Greiner, Adv. Mater. 19 (2007) 2480-2482, http://doi.org/10.1002/adma.200602936.

[23] H. Chen, Y. Liu, Q. Hu, Exp. Cell Res. 338 (2015) 261-266, http://doi.org/10.1016/j.yexcr.2015.08.007.

[24] H. Zhang, J. Bioact. Compat. Polym. 26 (2011) 590-606, http://doi.org/10.1177/0883911511424015.

[25] S.M. Feng, X. Liang Liu, J. Qi, D.L. Huang, Z.C. Xiong, Mater. Res. Express 6 (2019) 125330, http://doi.org/10.1088/2053-1591/ab58e0.

[26] S. Yu, Q. Huang, J. Cheng, Y. Huang, C. Xiao, Sep. Purif. Technol. 251 (2020) 117297, http://doi.org/10.1016/j.seppur.2020.117297. 
[27] F. Madani, R. Didekhani, M.R. Sohrabi, S. Mofavvaz, Bull. Mater. Sci. 43 (2020) 159, http://doi.org/10.1007/s12034-020-02138-y.

[28] P. Gupta, C. Elkins, T.E. Long, G.L. Wilkes, Polymer (Guildf). 46 (2005) 4799-4810, http://doi.org/10.1016/j.polymer.2005.04.021.

[29] J. Jiang, G. Zheng, X. Wang, W. Li, G. Kang, H. Chen, S. Guo, J. Liu, Micromachines 11 (2020) 27, http://doi.org/10.3390/mi11010027.

[30] Y. Yang, Z. Jia, Q. Li, L. Hou, J. Liu, L. Wang, Z. Guan, M. Zahn, IEEE Trans. Dielectr. Electr. Insul. 17 (2010) 1592-1601, http://doi.org/10.1109/TDEI.2010.5595562.

[31] S. Xie, Y. Zeng, Ind. Eng. Chem. Res. 51 (2012) 5336-5345, http://doi.org/10.1021/ie2020763.

[32] Y. Lu, X. Xiao, J. Fu, C. Huan, S. Qi, Y. Zhan, Y. Zhu, G. Xu, Chem. Eng. J. 355 (2019) 532-539, http://doi.org/10.1016/j.cej.2018.08.189.

[33] A.K. Moghe, B.S. Gupta, Polym. Rev. 48 (2008) 353-377, http://doi.org/10.1080/15583720802022257.

[34] A. Khalf, S. V. Madihally, Eur. J. Pharm. Biopharm. 112 (2017) 1-17, http://doi.org/10.1016/j.ejpb.2016.11.010.

[35] A. Katoch, S.S. Kim, J. Am. Ceram. Soc. 95 (2012) 553-556, http://doi.org/10.1111/j.1551-2916.2011.04923.x.

[36] M. Zhang, J. Chen, B. Chen, J. Cao, M. Hong, C. Zhou, Q. Xu, Appl. Surf. Sci. 367 (2016) 126-133, http://doi.org/10.1016/j.apsusc.2016.01.033.

[37] R. Halaui, E. Zussman, R. Khalfin, R. Semiat, Y. Cohen, Polym. Adv. Technol. 28 (2017) 570-582, http://doi.org/10.1002/pat.3794.

[38] D. Li, J.T. McCann, Y. Xia, Small 1 (2005) 83-86, http://doi.org/10.1002/smll.200400056.

[39] W. Yu, Q. Ma, X. Li, X. Dong, J. Wang, G. Liu, Mater. Lett. 120 (2014) 126-129, http://doi.org/10.1016/j.matlet.2014.01.076. 
[40] Q. Ma, J. Wang, X. Dong, W. Yu, G. Liu, RSC Adv. 5 (2015) 2523-2530, http://doi.org/10.1039/c4ra14908d.

[41] Z.C. Yao, C. Zhang, Z. Ahmad, J. Huang, J.S. Li, M.W. Chang, Chem. Eng. J. 334 (2018) 89-98, http://doi.org/10.1016/j.cej.2017.10.033.

[42] D.G. Yu, W. Chian, X. Wang, X.Y. Li, Y. Li, Y.Z. Liao, J. Memb. Sci. 428 (2013) 150-156, http://doi.org/10.1016/j.memsci.2012.09.062.

[43] D.G. Yu, K. White, J.H. Yang, X. Wang, W. Qian, Y. Li, Mater. Lett. 67 (2012) 78 80, http://doi.org/10.1016/j.matlet.2011.09.035.

[44] J.M. Yang, D.G. Yu, J. Polym. Res. 19 (2012) 1-7, http://doi.org/10.1007/s10965-0119789-x.

[45] D.-G. Yu, C.J. Branford-White, N.P. Chatterton, K. White, L.-M. Zhu, X.-X. Shen, W. Nie, Macromolecules 43 (2010) 10743, http://doi.org/10.1021/ma1024363.

[46] D. Han, A.J. Steckl, Langmuir 25 (2009) 9454-9462, http://doi.org/10.1021/la900660v.

[47] J. Wu, N. Wang, Y. Zhao, L. Jiang, J. Mater. Chem. A 1 (2013) 7290-7305, http://doi.org/10.1039/c3ta10451f.

[48] B. Kim, J. Kim, M. Kim, B.-D. Kim, S. Jo, S. Park, J. Son, S. Hwang, S. Dugasani, I. Chang, M. Kim, W. Liu, Nanotechnology 10 (2016) 105601, http://doi.org/10.1088/0957-4484.

[49] K. Nakata, S. Kinugawa, M. Takasaki, Y. Ohkoshi, Y. Gotoh, M. Nagura, FIBER 65 (2009) 257-261, http://doi.org/10.2115/fiber.65.257.

[50] S.N. Malakhov, S.N. Chvalun, Mater. Today Commun. 21 (2019) 100729, http://doi.org/10.1016/j.mtcomm.2019.100729.

[51] N. Ogata, S. Yamaguchi, N. Shimada, G. Lu, T. Iwata, K. Nakane, T. Ogihara, J. Appl. Polym. Sci. 104 (2007) 1640-1645, http://doi.org/10.1002/app.25782.

[52] M. Takasaki, K. Hara, Y. Ohkoshi, T. Fujii, H. Shimizu, M. Saito, Polym. Eng. Sci. 54 (2014) 2605-2609, http://doi.org/10.1002/pen.23811. 
[53] E. Zhmayev, D. Cho, Y.L. Joo, Polymer (Guildf). 51 (2010) 4140-4144, http://doi.org/10.1016/j.polymer.2010.06.058.

[54] Y. Ibrahim, E. Hussein, M. Zagho, G. Abdo, A. Elzatahry, Int. J. Mol. Sci. 20 (2019) 2455, http://doi.org/10.3390/ijms20102455.

[55] Y. Kadomae, Y. Maruyama, M. Sugimoto, T. Taniguchi, K. Koyama, Fibers Polym. 10 (2009) 275-279, http://doi.org/10.1007/s12221-009-0275-6.

[56] M.L. Muerza-Cascante, D. Haylock, D.W. Hutmacher, P.D. Dalton, Tissue Eng. - Part B Rev. 21 (2015) 187-202, http://doi.org/10.1089/ten.teb.2014.0347.

[57] S.R. Givens, K.H. Gardner, J.F. Rabolt, D.B. Chase, Macromolecules 40 (2007) 608610, http://doi.org/10.1021/ma062398a.

[58] Y.W. Cheng, H.A. Lu, Y.C. Wang, A. Thierry, B. Lotz, C. Wang, Macromolecules 43 (2010) 2371-2376, http://doi.org/10.1021/ma9025338.

[59] O. Jirsak, P. Sysel, F. Sanetrnik, J. Hruza, J. Chaloupek, J. Nanomater. 2010 (2010), http://doi.org/10.1155/2010/842831.

[60] S. Moon, R.J. Farris, Polym. Eng. Sci. 49 (2009) 1616-1620, http://doi.org/10.1002/pen.21355.

[61] G.Z. Yang, H.P. Li, J.H. Yang, J. Wan, D.G. Yu, Nanoscale Res. Lett. 12 (2017), http://doi.org/10.1186/s11671-016-1824-8.

[62] E.R. Lee, J.W. Cho, RSC Adv. 5 (2015) 78476-78482, http://doi.org/10.1039/c5ra14276h.

[63] T.L. Chang, C.H. Huang, S.Y. Chou, S.F. Tseng, Y.W. Lee, Microelectron. Eng. 177 (2017) 52-58, http://doi.org/10.1016/j.mee.2017.01.036.

[64] E. Adomavičiūtè, T. Tamulevičius, L. Šimatonis, E. Fataraitė-Urbonienè, E. Stankevičius, S. Tamulevičius, Medziagotyra 21 (2015) 44-51, http://doi.org/10.5755/j01.ms.21.1.10249.

[65] K.M. Forward, G.C. Rutledge, Chem. Eng. J. 183 (2012) 492-503, http://doi.org/10.1016/j.cej.2011.12.045. 
[66] B.K. Brettmann, S. Tsang, K.M. Forward, G.C. Rutledge, A.S. Myerson, B.L. Trout, Langmuir 28 (2012) 9714-9721, http://doi.org/10.1021/la301422x.

[67] K. Chansaengsri, K. Onlaor, B. Tunhoo, T. Thiwawong, in: Mater. Today Proc., Elsevier Ltd, 2017, pp. 6085-6090, http://doi.org/10.1016/j.matpr.2017.06.098.

[68] M.B. Bazbouz, H. Liang, G. Tronci, Mater. Sci. Eng. C 91 (2018) 541-555, http://doi.org/10.1016/j.msec.2018.05.076.

[69] D. Nurwaha, X. Wang, Fibers Polym. 16 (2015) 850-866, http://doi.org/10.1007/s12221-015-0850-y.

[70] Z. Liu, K. Keong, J. Ang, J. He, J. Mater. Sci. 52 (n.d.), http://doi.org/10.1007/s10853016-0472-9.

[71] G.T.V. Prabu, B. Dhurai, Sci. Rep. 10 (2020) 1-11, http://doi.org/10.1038/s41598020-60752-6.

[72] Y. Fang, L. Xu, Beilstein J. Nanotechnol. 10 (2019) 2261-2274, http://doi.org/10.3762/bjnano.10.218.

[73] Z. Shao, L. Yu, L. Xu, M. Wang, Nanoscale Res. Lett. 12 (2017) 470, http://doi.org/10.1186/s11671-017-2240-4.

[74] G. Jiang, X. Qin, Mater. Lett. 128 (2014) 259-262, http://doi.org/10.1016/j.matlet.2014.04.074.

[75] G. Jiang, S. Zhang, Y. Wang, X. Qin, Mater. Lett. 144 (2015) 22-25, http://doi.org/10.1016/j.matlet.2014.12.139.

[76] X.X. He, J. Zheng, G.F. Yu, M.H. You, M. Yu, X. Ning, Y.Z. Long, J. Phys. Chem. C 121 (2017) 8663-8678, http://doi.org/10.1021/acs.jpcc.6b12783.

[77] G. Zheng, J. Jiang, D. Wu, D. Sun, in: Electrospinning Nanofabrication Appl., 2019, pp. 283-319.

[78] K.P. Feltz, E.A. Growney Kalaf, C. Chen, R.S. Martin, S.A. Sell, Electrospinning 2 (2017) 46-61, http://doi.org/10.1515/esp-2017-0002. 
[79] Y. Liu, X. Zhang, Y. Xia, H. Yang, Adv. Mater. 22 (2010) 2454-2457, http://doi.org/10.1002/adma.200903870.

[80] T. Blachowicz, A. Ehrmann, J. Eng. Fiber. Fabr. 15 (2020), http://doi.org/10.1177/1558925019900843.

[81] K. Kim, J. Kim, H. Shim, Fibers Polym. 18 (2017) 493-501, http://doi.org/10.1007/s12221-017-5617-1.

[82] S. Basu, A.K. Agrawal, M. Jassal, J. Appl. Polym. Sci. 122 (2011) 856-866, http://doi.org/10.1002/app.34083.

[83] H.-W. Tong, M. Wang, Z.-Y. Li, al -, L.-X. Lü, Y.-Y. Wang, X. Mao, Biomed. Mater. 5 (2010) 054110, http://doi.org/10.1088/1748-6041/5/5/054110.

[84] C.J. Luo, E. Stride, M. Edirisinghe, Macromolecules 45 (2012) 4669-4680, http://doi.org/10.1021/ma300656u.

[85] H. He, Y. Kara, K. Molnár, Macromol. Mater. Eng. 304 (2019) 1900349 , http://doi.org/10.1002/mame.201900349.

[86] S.N. Patra, A.J. Easteal, D. Bhattacharyya, J. Mater. Sci. 44 (2009) 647-654, http://doi.org/10.1007/s10853-008-3050-y.

[87] P. Korycka, A. Mirek, K. Kramek-Romanowska, M. Grzeczkowicz, D. Lewińska, Beilstein J. Nanotechnol. 9 (2018) 2466-2478, http://doi.org/10.3762/bjnano.9.231.

[88] C.-M. Wu, H.-G. Chiou, S.-L. Lin, J.-M. Lin, J. Appl. Polym. Sci. 126 (2012) E89E97, http://doi.org/10.1002/app.36680.

[89] A. Kilic, F. Oruc, A. Demir, Text. Res. J. 78 (2008) 532-539, http://doi.org/10.1177/0040517507081296.

[90] U. Ali, X. Wang, T. Lin, J. Text. Inst. 103 (2012) 1160-1168, http://doi.org/10.1080/00405000.2012.664869.

[91] M.D.R. Kok, J.T. Gostick, J. Memb. Sci. 473 (2015) 237-244, http://doi.org/10.1016/j.memsci.2014.09.017. 
[92] O. Urbanek, P. Sajkiewicz, F. Pierini, Polymer (Guildf). 124 (2017) 168-175, http://doi.org/10.1016/j.polymer.2017.07.064.

[93] U. Stachewicz, C.A. Stone, C.R. Willis, A.H. Barber, J. Mater. Chem. 22 (2012) 22935-22941, http://doi.org/10.1039/c2jm33807f.

[94] F.O. Ochanda, M.A. Samaha, H.V. Tafreshi, G.C. Tepper, M. Gad-el-Hak, J. Appl. Polym. Sci. 123 (2012) 1112-1119, http://doi.org/10.1002/app.34583.

[95] S. Sarkar, S. Deevi, G. Tepper, Macromol. Rapid Commun. 28 (2007) 1034-1039, http://doi.org/10.1002/marc.200700053.

[96] S.P. Shariatpanahi, A. Iraji Zad, I. Abdollahzadeh, R. Shirsavar, D. Bonn, R. Ejtehadi, Soft Matter 7 (2011) 10548-10551, http://doi.org/10.1039/c1sm06009k.

[97] H.W. Tong, M. Wang, Mater. Lett. 94 (2013) 116-120, http://doi.org/10.1016/j.matlet.2012.12.015.

[98] P.S. Ginestra, S. Pandini, A. Fiorentino, P. Benzoni, P. Dell'Era, E. Ceretti, CIRP J. Manuf. Sci. Technol. 19 (2017) 147-157, http://doi.org/10.1016/j.cirpj.2017.08.002.

[99] G.H. Kim, H. Nam, W. Choi, T. An, G. Lim, Adv. Mater. Interfaces 5 (2018) 1701204, http://doi.org/10.1002/admi.201701204.

[100] C. Vaquette, J.J. Cooper-White, Acta Biomater. 7 (2011) 2544-2557, http://doi.org/10.1016/j.actbio.2011.02.036.

[101] P. Katta, M. Alessandro, R.D. Ramsier, G.G. Chase, Nano Lett. 4 (2004) 2215-2218, http://doi.org/10.1021/n10486158.

[102] Y. Zhou, G.Z. Tan, Nanomater. Nanotechnol. 7 (2017) 184798041774847, http://doi.org/10.1177/1847980417748478.

[103] C. Pan, Y. Han, L. Dong, J. Wang, Z. Gu, J. Macromol. Sci. Part B 47 (2008) 735742, http://doi.org/10.1080/00222340802118382.

[104] G. Chang, J. Shen, Macromol. Rapid Commun. 31 (2010) 2151-2154, http://doi.org/10.1002/marc.201000463. 
[105] J. Rafique, J. Yu, J. Yu, G. Fang, K.W. Wong, Z. Zheng, H.C. Ong, W.M. Lau, Appl. Phys. Lett. 91 (2007) 063126, http://doi.org/10.1063/1.2768871.

[106] X. Yan, M. Yu, W.P. Han, M.H. You, J.C. Zhang, R.H. Dong, H. Di Zhang, Y.Z. Long, Chinese Phys. B 25 (2016), http://doi.org/10.1088/1674-1056/25/7/078106.

[107] D.K. Dempsey, C.J. Schwartz, R.S. Ward, A. V. Iyer, J.P. Parakka, E.M. CosgriffHernandez, Macromol. Mater. Eng. 295 (2010) 990-994, http://doi.org/10.1002/mame.201000152.

[108] N. Shah Hosseini, N. Bölgen, N. Khenoussi, Ş.N. Yılmaz, D. Yetkin, A.H. Hekmati, L. Schacher, D. Adolphe, Int. J. Polym. Mater. Polym. Biomater. 67 (2018) 143-150, http://doi.org/10.1080/00914037.2017.1309541.

[109] M.N. Shuakat, T. Lin, J. Text. Inst. 107 (2016) 791-799, http://doi.org/10.1080/00405000.2015.1061785.

[110] F. Hejazi, H. Mirzadeh, N. Contessi, M.C. Tanzi, S. Faré, J. Biomed. Mater. Res. Part A 105 (2017) 1535-1548, http://doi.org/10.1002/jbm.a.35822.

[111] M.D.R. Kok, R. Jervis, D. Brett, P.R. Shearing, J.T. Gostick, Small 14 (2018) 1-15, http://doi.org/10.1002/smll.201703616.

[112] C. Blanc, A. Rufer, Paths to Sustain. Energy (2010), http://doi.org/10.5772/13338.

[113] Á. Cunha, J. Martins, N. Rodrigues, F.P. Brito, (2015) 889-918, http://doi.org/10.1002/er.

[114] G. Wei, J. Liu, H. Zhao, C. Yan, J. Power Sources 241 (2013) 709-717, http://doi.org/10.1016/j.jpowsour.2013.05.008.

[115] C. Flox, C. Fàbrega, T. Andreu, A. Morata, M. Skoumal, J. Rubio-Garcia, J.R. Morante, RSC Adv. 3 (2013) 12056-12059, http://doi.org/10.1039/c3ra40463c.

[116] G. Wei, Z. Gao, Z. Wei, X. Fan, J. Liu, C. Yan, Phys. Chem. Chem. Phys. 17 (2015) 20368-20375, http://doi.org/10.1039/c5cp02952j.

[117] C. Xu, X. Yang, X. Li, T. Liu, H. Zhang, J. Energy Chem. 26 (2017) 730-737, http://doi.org/10.1016/j.jechem.2017.03.005. 
[118] G. Wei, X. Fan, J. Liu, C. Yan, J. Power Sources 270 (2014) 634-645, http://doi.org/10.1016/j.jpowsour.2014.07.161.

[119] G. Wei, W. Su, Z. Wei, M. Jing, X. Fan, J. Liu, C. Yan, Electrochim. Acta 199 (2016) 147-153, http://doi.org/10.1016/j.electacta.2016.03.154.

[120] G. Wei, X. Fan, J. Liu, C. Yan, J. Power Sources 281 (2015) 1-6, http://doi.org/10.1016/j.jpowsour.2015.01.161.

[121] Z. He, M. Li, Y. Li, J. Zhu, Y. Jiang, W. Meng, H. Zhou, L. Wang, L. Dai, Electrochim. Acta 281 (2018) 601-610, http://doi.org/10.1016/j.electacta.2018.06.011.

[122] Z. He, M. Li, Y. Li, C. Li, Z. Yi, J. Zhu, L. Dai, W. Meng, H. Zhou, L. Wang, Electrochim. Acta 309 (2019) 166-176, http://doi.org/10.1016/j.electacta.2019.04.100.

[123] M. Jing, Z. Xu, D. Fang, X. Fan, J. Liu, C. Yan, J. Power Sources 425 (2019) 94-102, http://doi.org/10.1016/j.jpowsour.2019.04.003.

[124] M. Jing, X. Zhang, X. Fan, L. Zhao, J. Liu, C. Yan, Electrochim. Acta 215 (2016) 57 65, http://doi.org/10.1016/j.electacta.2016.08.095.

[125] C. Busacca, O. Di Blasi, N. Briguglio, M. Ferraro, V. Antonucci, A. Di Blasi, Electrochim. Acta 230 (2017) 174-180, http://doi.org/10.1016/j.electacta.2017.01.193.

[126] A. Di Blasi, C. Busaccaa, O. Di Blasia, N. Briguglio, G. Squadrito, V. Antonuccia, Appl. Energy 190 (2017) 165-171, http://doi.org/10.1016/j.apenergy.2016.12.129.

[127] A. Di Blasi, C. Busacca, O. Di Blasi, N. Briguglio, V. Antonucci, J. Electrochem. Soc. 165 (2018) A1478-A1485, http://doi.org/10.1149/2.1081807jes.

[128] G. Wei, M. Jing, X. Fan, J. Liu, C. Yan, J. Power Sources 287 (2015) 81-86, http://doi.org/10.1016/j.jpowsour.2015.04.041.

[129] M.D.R. Kok, A. Khalifa, J.T. Gostick, J. Electrochem. Soc. 163 (2016) A1408-A1419, http://doi.org/10.1149/2.1281607jes.

[130] S. Liu, M. Kok, Y. Kim, J.L. Barton, F.R. Brushett, J. Gostick, J. Electrochem. Soc. 164 (2017) A2038-A2048, http://doi.org/10.1149/2.1301709jes. 
[131] M.A. Sadeghi, M. Aganou, M. Kok, M. Aghighi, G. Merle, J. Barralet, J. Gostick, J. Electrochem. Soc. 166 (2019) A2121-A2130, http://doi.org/10.1149/2.0721910jes.

[132] R. Jervis, M.D.R. Kok, J. Montagut, J.T. Gostick, D.J.L. Brett, P.R. Shearing, Energy Technol. 6 (2018) 2488-2500, http://doi.org/10.1002/ente.201800338.

[133] M.D.R. Kok, R. Jervis, T.G. Tranter, M.A. Sadeghi, D.J.L. Brett, P.R. Shearing, J.T. Gostick, Chem. Eng. Sci. 196 (2019) 104-115, http://doi.org/10.1016/j.ces.2018.10.049.

[134] D. Zhang, A. Forner-Cuenca, O.O. Taiwo, V. Yufit, F.R. Brushett, N.P. Brandon, S. Gu, Q. Cai, J. Power Sources 447 (2020) 227249, http://doi.org/10.1016/j.jpowsour.2019.227249.

[135] Q. Wu, Y. Lv, L. Lin, X. Zhang, Y. Liu, X. Zhou, J. Power Sources 410-411 (2019) 152-161, http://doi.org/10.1016/j.jpowsour.2018.11.020.

[136] Z. He, M. Li, Y. Li, L. Wang, J. Zhu, W. Meng, C. Li, H. Zhou, L. Dai, Appl. Surf. Sci. 469 (2019) 423-430, http://doi.org/10.1016/j.apsusc.2018.10.220.

[137] A.K. Singh, N. Yasri, K. Karan, E.P.L. Roberts, ACS Appl. Energy Mater. 2 (2019) 2324-2336, http://doi.org/10.1021/acsaem.9b00180.

[138] B. Sun, M. Skyllas-Kazacos, Electrochim. Acta 37 (1992) 1253-1260, http://doi.org/10.1016/0013-4686(92)85064-R.

[139] K.J. Kim, M.S. Park, J.H. Kim, U. Hwang, N.J. Lee, G. Jeong, Y.J. Kim, Chem. Commun. 48 (2012) 5455-5457, http://doi.org/10.1039/c2cc31433a.

[140] L.D. Brown, T.P. Neville, R. Jervis, T.J. Mason, P.R. Shearing, D.J.L. Brett, J. Energy Storage 8 (2016) 91-98, http://doi.org/10.1016/j.est.2016.10.003.

[141] R. Jervis, M.D.R. Kok, T.P. Neville, Q. Meyer, L.D. Brown, F. Iacoviello, J.T. Gostick, D.J.L. Brett, P.R. Shearing, J. Energy Chem. 27 (2018) 1353-1361, http://doi.org/10.1016/j.jechem.2018.03.022.

[142] P. Trogadas, O.O. Taiwo, B. Tjaden, T.P. Neville, S. Yun, J. Parrondo, V. Ramani, M.O. Coppens, D.J.L. Brett, P.R. Shearing, Electrochem. Commun. 48 (2014) 155- 
159, http://doi.org/10.1016/j.elecom.2014.09.010.

[143] A. Fetyan, J. Schneider, M. Schnucklake, G.A. El-Nagar, R. Banerjee, N. Bevilacqua, R. Zeis, C. Roth, ChemElectroChem 6 (2019) 130-135, http://doi.org/10.1002/celc.201801128.

[144] R. Banerjee, N. Bevilacqua, L. Eifert, R. Zeis, J. Energy Storage 21 (2019) 163-171, http://doi.org/10.1016/j.est.2018.11.014.

[145] Y. Huang, F. Cui, Y. Zhao, J. Bao, J. Lian, Y. Xu, T. Liu, H. Li, Chem. - An Asian J. 12 (2017) 2426-2433, http://doi.org/10.1002/asia.201700750.

[146] J. Sun, L. Zeng, H.R. Jiang, C.Y.H. Chao, T.S. Zhao, J. Power Sources 405 (2018) 106-113, http://doi.org/10.1016/j.jpowsour.2018.10.035.

[147] C. Xu, X. Li, T. Liu, H. Zhang, RSC Adv. 7 (2017) 45932-45937, http://doi.org/10.1039/c7ra07365h.

[148] Z. Zhang, B. Bai, L. Zeng, L. Wei, T. Zhao, Energy Technol. 7 (2019) 1-8, http://doi.org/10.1002/ente.201900488.

[149] Z. Xu, W. Xiao, K. Zhang, D. Zhang, H. Wei, X. Zhang, Z. Zhang, N. Pu, J. Liu, C. Yan, J. Power Sources 450 (2020) 227686, http://doi.org/10.1016/j.jpowsour.2019.227686.

[150] Y. Zhou, J. Ren, Y. Yang, Q. Zheng, J. Liao, F. Xie, W. Jie, D. Lin, J. Solid State Chem. 268 (2018) 149-158, http://doi.org/10.1016/j.jssc.2018.08.041.

[151] S. Hérou, M. Crespo Ribadeneyra, P. Schlee, H. Luo, L.C. Tanase, C. Roßberg, M. Titirici, J. Energy Chem. 53 (2020) 36-48, http://doi.org/10.1016/j.jechem.2020.04.068.

[152] A.J. Ragauskas, G.T. Beckham, M.J. Biddy, R. Chandra, F. Chen, M.F. Davis, B.H. Davison, R.A. Dixon, P. Gilna, M. Keller, P. Langan, A.K. Naskar, J.N. Saddler, T.J. Tschaplinski, G.A. Tuskan, C.E. Wyman, Science (80-. ). 344 (2014), http://doi.org/10.1126/science.1246843.

[153] J.F. Vivo-Vilches, A. Celzard, V. Fierro, I. Devin-Ziegler, N. Brosse, A. Dufour, M. 
Etienne, Nanomaterials 9 (2019) 1-13, http://doi.org/10.3390/nano9010106.

[154] M.C. Ribadeneyra, L. Grogan, H. Au, P. Schlee, S. Herou, T. Neville, P.L. Cullen, M.D.R. Kok, O. Hosseinaei, S. Danielsson, P. Tomani, M.M. Titirici, D.J.L. Brett, P.R. Shearing, R. Jervis, A.B. Jorge, Carbon N. Y. 157 (2020) 847-856, http://doi.org/10.1016/j.carbon.2019.11.015.

[155] M. Skyllas-Kazacos, M.H. Chakrabarti, S.A. Hajimolana, F.S. Mjalli, M. Saleem, J. Electrochem. Soc. 158 (2011) R55, http://doi.org/10.1149/1.3599565.

[156] S. Sui, X. Wang, X. Zhou, Y. Su, S. Riffat, C. jun Liu, J. Mater. Chem. A 5 (2017) 1808-1825, http://doi.org/10.1039/C6TA08580F.

[157] S. Cavaliere, I. Jiménez-Morales, G. Ercolano, I. Savych, D. Jones, J. Rozière, ChemElectroChem 2 (2015) 1966-1973, http://doi.org/10.1002/celc.201500330.

[158] I. Savych, S. Subianto, Y. Nabil, S. Cavaliere, D. Jones, J. Rozière, Phys. Chem. Chem. Phys. 17 (2015) 16970-16976, http://doi.org/10.1039/c5cp01542a.

[159] G. Cognard, G. Ozouf, C. Beauger, I.J. Morales, S. Cavaliere, D. Jones, J. Roziere, M. Chatenet, F. Maillard, Electrocatalysis 8 (2016) 51-58.

[160] Y. Nabil, S. Cavaliere, I.A. Harkness, J.D.B. Sharman, D.J. Jones, J. Rozière, J. Power Sources 363 (2017) 20-26, http://doi.org/10.1016/j.jpowsour.2017.07.058.

[161] J. Li, S. Brüller, D.C. Sabarirajan, N. Ranjbar-Sahraie, M.T. Sougrati, S. Cavaliere, D. Jones, I. V. Zenyuk, A. Zitolo, F. Jaouen, ACS Appl. Energy Mater. 2 (2019) 72117222, http://doi.org/10.1021/acsaem.9b01181.

[162] Y. Wang, J. Jin, S. Yang, G. Li, J. Jiang, Int. J. Hydrogen Energy 41 (2016) 1117411184, http://doi.org/10.1016/j.ijhydene.2016.04.235.

[163] A. Jindal, S. Basu, C.P. Aby, RSC Adv. 5 (2015) 69378-69387, http://doi.org/10.1039/c5ra10884e.

[164] T. Gong, R. Qi, X. Liu, H. Li, Y. Zhang, Nano-Micro Lett. 11 (2019) 1-11, http://doi.org/10.1007/s40820-019-0240-x.

[165] M.F. Mathias, J. Roth, J. Fleming, W. Lehnert, Handb. Fuel Cells 3 (2010), 
http://doi.org/10.1002/9780470974001.f303046.

[166] W.R. Purcell, J. Pet. Technol. 2 (1950) 11-12, http://doi.org/10.2118/950369-g.

[167] T.G. Tranter, J.T. Gostick, A.D. Burns, W.F. Gale, Transp. Porous Media 121 (2018) 597-620, http://doi.org/10.1007/s11242-017-0973-2.

[168] D. Todd, W. Mérida, J. Power Sources 273 (2015) 312-316, http://doi.org/10.1016/j.jpowsour.2014.09.095.

[169] D. Todd, W. Mérida, J. Power Sources 311 (2016) 182-187, http://doi.org/10.1016/j.jpowsour.2016.02.031.

[170] S. Chevalier, N. Lavielle, B.D. Hatton, A. Bazylak, J. Power Sources 352 (2017) 272280, http://doi.org/10.1016/j.jpowsour.2017.03.098.

[171] S. Chevalier, N. Ge, J. Lee, M.G. George, H. Liu, P. Shrestha, D. Muirhead, N. Lavielle, B.D. Hatton, A. Bazylak, J. Power Sources 352 (2017) 281-290, http://doi.org/10.1016/j.jpowsour.2017.01.114.

[172] M. Salahuddin, M.N. Uddin, G. Hwang, R. Asmatulu, Int. J. Hydrogen Energy 43 (2018) 11530-11538, http://doi.org/10.1016/j.ijhydene.2017.07.229.

[173] Q. Duan, B. Wang, J. Wang, H. Wang, Y. Lu, J. Power Sources 195 (2010) 81898193, http://doi.org/10.1016/j.jpowsour.2010.07.032.

[174] C. Li, D. Si, Y. Liu, J. Zhang, Y. Liu, Int. J. Hydrogen Energy (2020) 1-10, http://doi.org/10.1016/j.ijhydene.2020.05.271.

[175] H.F. Lee, P.C. Wang, Y.W. Chen-Yang, J. Solid State Electrochem. 23 (2019) 971984, http://doi.org/10.1007/s10008-019-04198-5.

[176] R. Schweiss, M. Steeb, P.M. Wilde, Fuel Cells 10 (2010) 1176-1180, http://doi.org/10.1002/fuce.201000003.

[177] M. Balakrishnan, P. Shrestha, N. Ge, C. Lee, K.F. Fahy, R. Zeis, V.P. Schulz, B.D. Hatton, A. Bazylak, ACS Appl. Energy Mater. 3 (2020) 2695-2707, http://doi.org/10.1021/acsaem.9b02371. 
[178] Z. Li, X. Deng, H. Zhou, W. Xuan, Z. Xie, F. Liu, J. Solid State Electrochem. 24 (2020) 195-206, http://doi.org/10.1007/s10008-019-04474-4.

[179] X. Deng, S. Yin, X. Wu, M. Sun, Z. Xie, Q. Huang, Electrochim. Acta 283 (2018) 987-996, http://doi.org/10.1016/j.electacta.2018.06.139.

[180] M. Kim, C. Kwon, K. Eom, J. Kim, E. Cho, Sci. Rep. 7 (2017) 1-8, http://doi.org/10.1038/srep44411

[181] H. Kim, M.K. Cho, J.A. Kwon, Y.H. Jeong, K.J. Lee, N.Y. Kim, M.J. Kim, S.J. Yoo, J.H. Jang, H.J. Kim, S.W. Nam, D.H. Lim, E.A. Cho, K.Y. Lee, J.Y. Kim, Nanoscale 7 (2015) 18429-18434, http://doi.org/10.1039/c5nr04082e.

[182] N.Y. Kim, J.H. Lee, J.A. Kwon, S.J. Yoo, J.H. Jang, H.J. Kim, D.H. Lim, J.Y. Kim, J. Ind. Eng. Chem. 46 (2017) 298-303, http://doi.org/10.1016/j.jiec.2016.10.043.

[183] Y. Wang, G. Li, J. Jin, S. Yang, Int. J. Hydrogen Energy 42 (2017) 5938-5947, http://doi.org/10.1016/j.ijhydene.2017.02.012.

[184] K.K. Karuppanan, A. V. Raghu, M.K. Panthalingal, S. Ramanathan, T. Kumaresan, B. Pullithadathil, J. Mater. Chem. A 6 (2018) 12768-12781, http://doi.org/10.1039/c8ta02391c.

[185] K.K. Karuppanan, A. V. Raghu, M.K. Panthalingal, B. Pullithadathil, ChemElectroChem 6 (2019) 2029-2042, http://doi.org/10.1002/celc.201900065.

[186] S. Hong, M. Hou, Y. Zeng, Z. Shao, B. Yi, ChemElectroChem 4 (2017) 1007-1010, http://doi.org/10.1002/celc.201600841.

[187] M.A. Khalily, B. Patil, E. Yilmaz, T. Uyar, ChemNanoMat 5 (2019) 1540-1546, http://doi.org/10.1002/cnma.201900483.

[188] Y. Shao, J.P. Dodelet, G. Wu, P. Zelenay, Adv. Mater. 31 (2019) 1-8, http://doi.org/10.1002/adma.201807615.

[189] M. Sun, Z. Xie, Z. Li, X. Deng, Q. Huang, Z. Li, Int. J. Hydrogen Energy 44 (2019) 24617-24627, http://doi.org/10.1016/j.ijhydene.2019.07.182.

[190] B. Li, S.P. Sasikala, D.H. Kim, J. Bak, I.D. Kim, E.A. Cho, S.O. Kim, Nano Energy 56 
(2019) 524-530, http://doi.org/10.1016/j.nanoen.2018.11.054.

[191] H. Zou, S. Pei, Z. Zhou, Z. Chen, X. Xiong, Y. Sun, Y. Zhang, RSC Adv. 10 (2019) 779-783, http://doi.org/10.1039/c9ra08951a.

[192] A.B. Jorge, R. Jervis, A.P. Periasamy, M. Qiao, J. Feng, L.N. Tran, M.M. Titirici, Adv. Energy Mater. 10 (2020), http://doi.org/10.1002/aenm.201902494.

[193] L. Delmondo, J.A. Muñoz-Tabares, A. Sacco, N. Garino, G. Massaglia, M. Castellino, G.P. Salvador, C.F. Pirri, M. Quaglio, A. Chiodoni, Phys. Chem. Chem. Phys. 19 (2017) 28781-28787, http://doi.org/10.1039/c7cp05091g.

[194] Y.W. Lee, G.H. An, S. Lee, J. Hong, B.S. Kim, J. Lee, D.H. Kwak, H.J. Ahn, W. Huh, S.N. Cha, K.W. Park, J.I. Sohn, J.M. Kim, Catal. Sci. Technol. 6 (2016) 2085-2091, http://doi.org/10.1039/c5cy02016f.

[195] J. Shui, C. Chen, L. Grabstanowicz, D. Zhao, D.J. Liu, Proc. Natl. Acad. Sci. U. S. A. 112 (2015) 10629-10634, http://doi.org/10.1073/pnas.1507159112.

[196] X. Yan, K. Liu, X. Wang, T. Wang, J. Luo, J. Zhu, Nanotechnology 26 (2015), http://doi.org/10.1088/0957-4484/26/16/165401.

[197] R. Singhal, V. Kalra, ChemPhysChem 18 (2017) 223-229, http://doi.org/10.1002/cphc.201600771.

[198] Y.G. Lee, H.J. Ahn, Appl. Surf. Sci. 487 (2019) 389-397, http://doi.org/10.1016/j.apsusc.2019.05.095.

[199] H. Tang, W. Chen, J. Wang, T. Dugger, L. Cruz, D. Kisailus, Small 14 (2018), http://doi.org/10.1002/smll.201703459.

[200] A. Kakoria, B. Devi, A. Anand, A. Halder, R.R. Koner, S. Sinha-Ray, ACS Appl. Nano Mater. 2 (2019) 64-74, http://doi.org/10.1021/acsanm.8b01651.

[201] L. Song, J. Tang, T. Wang, C. Wu, Y. Ide, J. He, Y. Yamauchi, Chem. - A Eur. J. 25 (2019) 6807-6813, http://doi.org/10.1002/chem.201900197.

[202] Y. Huang, Y.E. Miao, H. Lu, T. Liu, Chem. - A Eur. J. 21 (2015) 10100-10108, http://doi.org/10.1002/chem.201500924. 
[203] L. Delmondo, G.P. Salvador, J.A. Muñoz-Tabares, A. Sacco, N. Garino, M. Castellino, M. Gerosa, G. Massaglia, A. Chiodoni, M. Quaglio, Appl. Surf. Sci. 388 (2016) 631639, http://doi.org/10.1016/j.apsusc.2016.03.224.

[204] D. Hassen, M.A. Shenashen, S.A. El-Safty, M.M. Selim, H. Isago, A. Elmarakbi, A. El-Safty, H. Yamaguchi, J. Power Sources 330 (2016) 292-303, http://doi.org/10.1016/j.jpowsour.2016.08.140.

[205] A.R. Zeradjanin, ChemSusChem 11 (2018) 1247-1247, http://doi.org/10.1002/cssc.201800757.

[206] A. Bonakdarpour, M. Lefevre, R. Yang, F. Jaouen, T. Dahn, J.P. Dodelet, J.R. Dahn, Electrochem. Solid-State Lett. 11 (2008), http://doi.org/10.1149/1.2904768.

[207] E. Zussman, X. Chen, W. Ding, L. Calabri, D.A. Dikin, J.P. Quintana, R.S. Ruoff, Carbon N. Y. 43 (2005) 2175-2185, http://doi.org/10.1016/j.carbon.2005.03.031.

[208] J.S. Im, S.J. Park, Y.S. Lee, Mater. Res. Bull. 44 (2009) 1871-1878, http://doi.org/10.1016/j.materresbull.2009.05.010.

[209] J.S. Im, S.J. Park, T. Kim, Y.S. Lee, Int. J. Hydrogen Energy 34 (2009) 3382-3388, http://doi.org/10.1016/j.ijhydene.2009.02.047.

[210] H.F. Wang, Q. Xu, Matter 1 (2019) 565-595, http://doi.org/10.1016/j.matt.2019.05.008.

[211] D. Ji, S. Peng, D. Safanama, H. Yu, L. Li, G. Yang, X. Qin, M. Srinivasan, S. Adams, S. Ramakrishna, Chem. Mater. 29 (2017) 1665-1675, http://doi.org/10.1021/acs.chemmater.6b05056.

[212] S. Martinez Crespiera, D. Amantia, E. Knipping, C. Aucher, L. Aubouy, J. Amici, J. Zeng, C. Francia, S. Bodoardo, RSC Adv. 6 (2016) 57335-57345, http://doi.org/10.1039/c6ra09721a.

[213] J. Huang, B. Zhang, Y.Y. Xie, W.W.K. Lye, Z.L. Xu, S. Abouali, M. Akbari Garakani, J.Q. Huang, T.Y. Zhang, B. Huang, J.K. Kim, Carbon N. Y. 100 (2016) 329-336, http://doi.org/10.1016/j.carbon.2016.01.012. 
[214] J. Shim, K.J. Lopez, H.J. Sun, G. Park, J.C. An, S. Eom, S. Shimpalee, J.W. Weidner, J. Appl. Electrochem. 45 (2015) 1005-1012, http://doi.org/10.1007/s10800-015-08682.

[215] S. Hyun, S. Shanmugam, J. Power Sources 354 (2017) 48-56, http://doi.org/10.1016/j.jpowsour.2017.04.029.

[216] Y. Wang, J. Fu, Y. Zhang, M. Li, F.M. Hassan, G. Li, Z. Chen, Nanoscale 9 (2017) 15865-15872, http://doi.org/10.1039/c7nr05728h.

[217] S.W. Choi, Y.Z. Fu, Y.R. Ahn, S.M. Jo, A. Manthiram, J. Power Sources 180 (2008) 167-171, http://doi.org/10.1016/j.jpowsour.2008.02.042.

[218] M. Inagaki, K. Fujita, Y. Takeuchi, K. Oshida, H. Iwata, H. Konno, Carbon N. Y. 39 (2001) 921-929, http://doi.org/10.1016/S0008-6223(00)00210-4.

[219] L. Lai, J.R. Potts, D. Zhan, L. Wang, C.K. Poh, C. Tang, H. Gong, Z. Shen, J. Lin, R.S. Ruoff, Energy Environ. Sci. 5 (2012) 7936-7942, http://doi.org/10.1039/c2ee21802j.

[220] G. Wu, P. Zelenay, Acc. Chem. Res. 46 (2013) 1878-1889, http://doi.org/10.1021/ar400011z.

[221] J. Lu, Y. Lei, K.C. Lau, X. Luo, P. Du, J. Wen, R.S. Assary, U. Das, D.J. Miller, J.W. Elam, H.M. Albishri, D.A. El-Hady, Y.K. Sun, L.A. Curtiss, K. Amine, Nat. Commun. 4 (2013) 1-10, http://doi.org/10.1038/ncomms3383.

[222] S. Martinez Crespiera, D. Amantia, E. Knipping, C. Aucher, L. Aubouy, J. Amici, J. Zeng, U. Zubair, C. Francia, S. Bodoardo, J. Appl. Electrochem. 47 (2017) 497-506, http://doi.org/10.1007/s10800-016-1035-0.

[223] S. Wang, C. Dai, J. Li, L. Zhao, Z. Ren, Y. Ren, Y. Qiu, J. Yu, Int. J. Hydrogen Energy 40 (2015) 4673-4682, http://doi.org/10.1016/j.ijhydene.2015.02.031.

[224] Q. Liu, Y. Wang, L. Dai, J. Yao, Adv. Mater. 28 (2016) 3000-3006, http://doi.org/10.1002/adma.201506112.

[225] G.S. Park, J.S. Lee, S.T. Kim, S. Park, J. Cho, J. Power Sources 243 (2013) 267-273, 
http://doi.org/10.1016/j.jpowsour.2013.06.025.

[226] C.L. Huang, P.Y. Wang, Y.Y. Li, Chem. Eng. J. 395 (2020) 125031, http://doi.org/10.1016/j.cej.2020.125031.

[227] M.C. Kim, J.Y. So, S.H. Moon, S.B. Han, S. Choi, E.S. Kim, Y.K. Shin, J.E. Lee, D.H. Kwak, C. Lee, W.G. Bae, K.W. Park, J. Mater. Chem. A 6 (2018) 9550-9560, http://doi.org/10.1039/c8ta00281a.

[228] D.L. Boos, Electrolytic Capacitor Having Carbon Paste Electrodes, U.S. Patent $3536963,1970$.

[229] P. Azais, in: Supercapacitors Mater. Syst. Appl., 2013, pp. 307-372.

[230] T. Brousse, D. Bélanger, K. Chiba, M. Egashira, F. Favier, J. Long, J.R. Miller, M. Morita, K. Naoi, P. Simon, W. Sugimoto, in: Handb. Electrochem. Energy, 2017, pp. 495-561.

[231] J. Lelieveld, J.S. Evans, M. Fnais, D. Giannadaki, A. Pozzer, Nature 525 (2015) 367371, http://doi.org/10.1038/nature15371.

[232] L. Jin, Y. Zheng, J. Li, Y. Liu, (2015), http://doi.org/10.1177/1687814015573762.

[233] P. Simon, Y. Gogotsi, B. Dunn, Science (80-. ). 343 (2014) 1210-1211, http://doi.org/10.1126/science.1249625.

[234] L. Selley, L. Schuster, H. Marbach, T. Forsthuber, B. Forbes, T.W. Gant, T. Sandstrom, N. Camina, T.J. Athersuch, I. Mudway, A. Kumarb, Metallomics 12 (2020) 371-386, http://doi.org/10.1039/c9mt00253g.

[235] A. Tolosa, B. Krüner, S. Fleischmann, N. Jäckel, M. Zeiger, M. Aslan, I. Grobelsek, V. Presser, J. Mater. Chem. A 4 (2016) 16003-16016, http://doi.org/10.1039/c6ta06224e.

[236] F.J. García-Mateos, R. Berenguer, M.J. Valero-Romero, J. Rodríguez-Mirasol, T. Cordero, J. Mater. Chem. A 6 (2018) 1219-1233, http://doi.org/10.1039/c7ta08788h.

[237] J. Chmiola, C. Largeot, P.L. Taberna, P. Simon, Y. Gogotsi, Angew. Chemie - Int. Ed. 47 (2008) 3392-3395, http://doi.org/10.1002/anie.200704894. 
[238] C. Merlet, C. Péan, B. Rotenberg, P.A. Madden, B. Daffos, P.L. Taberna, P. Simon, M. Salanne, Nat. Commun. 4 (2013), http://doi.org/10.1038/ncomms3701.

[239] Y. Zhai, Y. Dou, D. Zhao, P.F. Fulvio, R.T. Mayes, S. Dai, Adv. Mater. 23 (2011) 4828-4850, http://doi.org/10.1002/adma.201100984.

[240] L. Guan, L. Yu, G.Z. Chen, Electrochim. Acta 206 (2016), http://doi.org/10.1016/j.electacta.2016.01.213.

[241] X. Zheng, J. Luo, W. Lv, D.-W. Wang, Q.-H. Yang, Adv. Mater. 27 (2015) 53885395, http://doi.org/10.1002/adma.201501452.

[242] L. Chang, Y.H. Hu, Matter 1 (2019) 596-620, http://doi.org/10.1016/j.matt.2019.06.016.

[243] R. Yan, M. Antonietti, M. Oschatz, Adv. Energy Mater. 8 (2018), http://doi.org/10.1002/aenm.201800026.

[244] L. Weinstein, R. Dash, Mater. Today 16 (2013) 356-357, http://doi.org/10.1016/j.mattod.2013.09.005

[245] P. Simon, A. Burke, Electrochem. Soc. Interface (2008) 38-43.

[246] A. Burke, Electrochim. Acta 53 (2007) 1083-1091, http://doi.org/10.1016/j.electacta.2007.01.011.

[247] X. Lu, M. Yu, G. Wang, Y. Tong, Y. Li, Energy Environ. Sci. 7 (2014) 2160-2181, http://doi.org/10.1039/c4ee00960f.

[248] J. Liang, H. Zhao, L. Yue, G. Fan, T. Li, S. Lu, (2020) 16747-16789, http://doi.org/10.1039/d0ta05100d.

[249] P. Schlee, S. Herou, R. Jervis, P.R. Shearing, D.J.L. Brett, D. Baker, O. Hosseinaei, P. Tomani, M.M. Murshed, Y. Li, M.J. Mostazo-López, D. Cazorla-Amorós, A.B. Jorge Sobrido, M.M. Titirici, Chem. Sci. 10 (2019) 2980-2988, http://doi.org/10.1039/c8sc04936j.

[250] S.R. Dods, O. Hardick, B. Stevens, D.G. Bracewell, J. Chromatogr. A 1376 (2015) 7483, http://doi.org/10.1016/j.chroma.2014.12.010. 
[251] J. Zhou, Z. Li, W. Xing, H. Shen, X. Bi, T. Zhu, Z. Qiu, (2016) 7955-7964, http://doi.org/10.1002/adfm.201601904.

[252] Z. Zhou, T. Liu, A.U. Khan, G. Liu, Mol. Syst. Des. Eng. 5 (2020) 153-165, http://doi.org/10.1039/c9me00066f.

[253] M.M. Pérez-Madrigal, M.G. Edo, C. Alemán, Green Chem. 18 (2016) 5930-5956, http://doi.org/10.1039/C6GC02086K.

[254] G.C. Loh, D. Baillargeat, J. Appl. Phys. 114 (2013), http://doi.org/10.1063/1.4816313.

[255] S. Ozbek, D.H. Isaac, Carbon N. Y. 38 (2000) 2007-2016, http://doi.org/10.1016/S0008-6223(00)00060-9.

[256] E. Frank, L.M. Steudle, D. Ingildeev, J.M. Spörl, M.R. Buchmeiser, Angew. Chemie Int. Ed. 53 (2014) 5262-5298, http://doi.org/10.1002/anie.201306129.

[257] M. Inagaki, M. Toyoda, T. Tsumura, RSC Adv. 40 (2014) 41411-41424, http://doi.org/10.1039/c4ra06730d.

[258] E. Frank, F. Hermanutz, M.R. Buchmeiser, Macromol. Mater. Eng. 297 (2012) $493-$ 501, http://doi.org/10.1002/mame.201100406.

[259] W. Fang, S. Yang, X.-L. Wang, T.-Q. Yuan, R.-C. Sun, Green Chem. 19 (2017) 17941827, http://doi.org/10.1039/c6gc03206k.

[260] Y. Zhou, J. Ren, L. Xia, Q. Zheng, J. Liao, E. Long, F. Xie, C. Xu, D. Lin, Electrochim. Acta 284 (2018) 336-345, http://doi.org/10.1016/j.electacta.2018.07.134.

[261] C. Kim, K.S. Yang, Appl. Phys. Lett. 83 (2003) 1216-1218, http://doi.org/10.1063/1.1599963.

[262] E.J. Ra, E. Raymundo-Pinero, Y.H. Lee, F. Béguin, Carbon N. Y. 47 (2009) 29842992, http://doi.org/10.1016/j.carbon.2009.06.051.

[263] B.H. Kim, K.S. Yang, Y.A. Kim, Y.J. Kim, B. An, K. Oshida, J. Power Sources 196 (2011) 10496-10501, http://doi.org/10.1016/j.jpowsour.2011.08.088.

[264] B.-H. Kim, K.S. Yang, H.-G. Woo, J. Nanosci. Nanotechnol. 11 (2011) 7193-7197, 
http://doi.org/10.1166/jnn.2011.4796.

[265] B.-H. Kim, K.S. Yang, H.-G. Woo, Electrochem. Commun. 13 (2011) 1042-1046, http://doi.org/10.1016/j.elecom.2011.06.024.

[266] A. Amiri, B. Conlee, I. Tallerine, W.J. Kennedy, M. Naraghi, Chem. Eng. J. 399 (2020), http://doi.org/10.1016/j.cej.2020.125788.

[267] S. Herou, P. Schlee, A.B. Jorge, M. Titirici, Curr. Opin. Green Sustain. Chem. 9 (2018) 18-24, http://doi.org/10.1016/j.cogsc.2017.10.005.

[268] P. Schlee, S. Herou, R. Jervis, P.R. Shearing, D.J.L. Brett, D. Baker, O. Hosseinaei, P. Tomani, M.M. Murshed, Y. Li, M.J. Mostazo-López, D. Cazorla-Amorós, A.B. Jorge Sobrido, M.M. Titirici, Chem. Sci. 10 (2019) 2980-2988, http://doi.org/https://doi.org/10.1039/C8SC04936J.

[269] F.J. García-Mateos, R. Ruiz-Rosas, J. María Rosas, E. Morallón, D. Cazorla-Amorós, J. Rodríguez-Mirasol, T. Cordero, Sep. Purif. Technol. 241 (2020), http://doi.org/10.1016/j.seppur.2020.116724.

[270] J. Ralph, K. Lundquist, G. Brunow, F. Lu, H. Kim, P.F. Schatz, J.M. Marita, R.D. Hatfield, S.A. Ralph, J.H. Christensen, W. Boerjan, Lignins: Natural Polymers from Oxidative Coupling of 4-Hydroxyphenyl-Propanoids, 2004.

[271] P. Schlee, O. Hosseinaei, D. Baker, A. Landmér, P. Tomani, M.J. Mostazo-López, D. Cazorla-Amorós, S. Herou, M.M. Titirici, Carbon N. Y. 145 (2019) 470-480, http://doi.org/10.1016/j.carbon.2019.01.035.

[272] R. Rinaldi, R. Jastrzebski, M.T. Clough, J. Ralph, M. Kennema, P.C.A. Bruijnincx, B.M. Weckhuysen, Angew. Chemie - Int. Ed. 55 (2016) 8164-8215, http://doi.org/10.1002/anie.201510351.

[273] A. Berlin, M. Balakshin, in: Bioenergy Res. Adv. Appl., Elsevier Inc., 2014, pp. 315336, http://doi.org/10.1016/B978-0-444-59561-4.00018-8.

[274] M. Lallave, J. Bedia, R. Ruiz-Rosas, J. Rodríguez-Mirasol, T. Cordero, J.C. Otero, M. Marquez, A. Barrero, I.G. Loscertales, Adv. Mater. 19 (2007) 4292-4296, http://doi.org/10.1002/adma.200700963. 
[275] R. Berenguer, F.J. García-Mateos, R. Ruiz-Rosas, D. Cazorla-Amorós, E. Morallón, J. Rodríguez-Mirasol, T. Cordero, Green Chem. 18 (2016) 1506-1515, http://doi.org/10.1039/C5GC02409A.

[276] A. Greiner, J.H. Wendorff, Angew. Chemie - Int. Ed. 46 (2007) 5670-5703, http://doi.org/10.1002/anie.200604646.

[277] F. Béguin, V. Presser, A. Balducci, E. Frackowiak, Adv. Mater. 26 (2014) 2219-2251, http://doi.org/10.1002/adma.201304137.

[278] J. Yang, Y. Wang, J. Luo, L. Chen, ACS Omega 3 (2018) 4647-4656, http://doi.org/10.1021/acsomega.7b01876.

[279] D. Wu, X. Xie, Y. Zhang, D. Zhang, W. Du, X. Zhang, B. Wang, Front. Mater. 7 (2020), http://doi.org/10.3389/fmats.2020.00002.

[280] D. Bélanger, T. Brousse, J.W. Long, Electrochem. Soc. Interface (2008) 49-52.

[281] J.-G. Wang, Y. Yang, Z.-H. Huang, F. Kang, Electrochim. Acta 75 (2012) 213-219.

[282] X. Ma, P. Kolla, Y. Zhao, A.L. Smirnova, H. Fong, J. Power Sources 325 (2016) 541548, http://doi.org/10.1016/j.jpowsour.2016.06.073.

[283] L. Zhang, Q. Ding, Y. Huang, H. Gu, Y.E. Miao, T. Liu, ACS Appl. Mater. Interfaces 7 (2015) 22669-22677, http://doi.org/10.1021/acsami.5b07528.

[284] G.M. Tomboc, H. Kim, Electrochim. Acta 318 (2019) 392-404, http://doi.org/10.1016/j.electacta.2019.06.112.

[285] P. Zhao, M. Yao, H. Ren, N. Wang, S. Komarneni, Appl. Surf. Sci. 463 (2019) 931938, http://doi.org/10.1016/j.apsusc.2018.09.041.

[286] X. Lang, A. Hirata, T. Fujita, M. Chen, Nat. Nanotechnol. 6 (2011) 232-236, http://doi.org/10.1038/nnano.2011.13.

[287] K.S. Kasprzak, F.W. Sunderman, K. Salnikow, Mutat. Res. - Fundam. Mol. Mech. Mutagen. 533 (2003) 67-97, http://doi.org/10.1016/j.mrfmmm.2003.08.021.

[288] F. Luan, G. Wang, Y. Ling, X. Lu, H. Wang, Y. Tong, X.X. Liu, Y. Li, Nanoscale 5 
(2013) 7984-7990, http://doi.org/10.1039/c3nr02710d.

[289] Y.Y. Peng, B. Akuzum, N. Kurra, M.Q. Zhao, M. Alhabeb, B. Anasori, E.C. Kumbur, H.N. Alshareef, M. Der Ger, Y. Gogotsi, Energy Environ. Sci. 9 (2016) 2847-2854, http://doi.org/10.1039/c6ee01717g.

[290] A.S. Levitt, M. Alhabeb, C.B. Hatter, A. Sarycheva, G. Dion, Y. Gogotsi, Electrospun MXene/Carbon Nanofibers as Supercapacitor Electrodes, 2018.

[291] Y. Li, H. Wang, L. Wang, Z. Mao, R. Wang, B. He, Y. Gong, 1804539 (2019) 1-13, http://doi.org/10.1002/smll.201804539.

[292] C. Ma, Y. Song, J. Shi, D. Zhang, X. Zhai, M. Zhong, Q. Guo, L. Liu, Carbon N. Y. 51 (2013) 290-300, http://doi.org/10.1016/j.carbon.2012.08.056.

[293] C. Zhang, X. Yuan, L. Wu, Y. Han, J. Sheng, Eur. Polym. J. 41 (2005) 423-432, http://doi.org/10.1016/j.eurpolymj.2004.10.027.

Graphical abstract

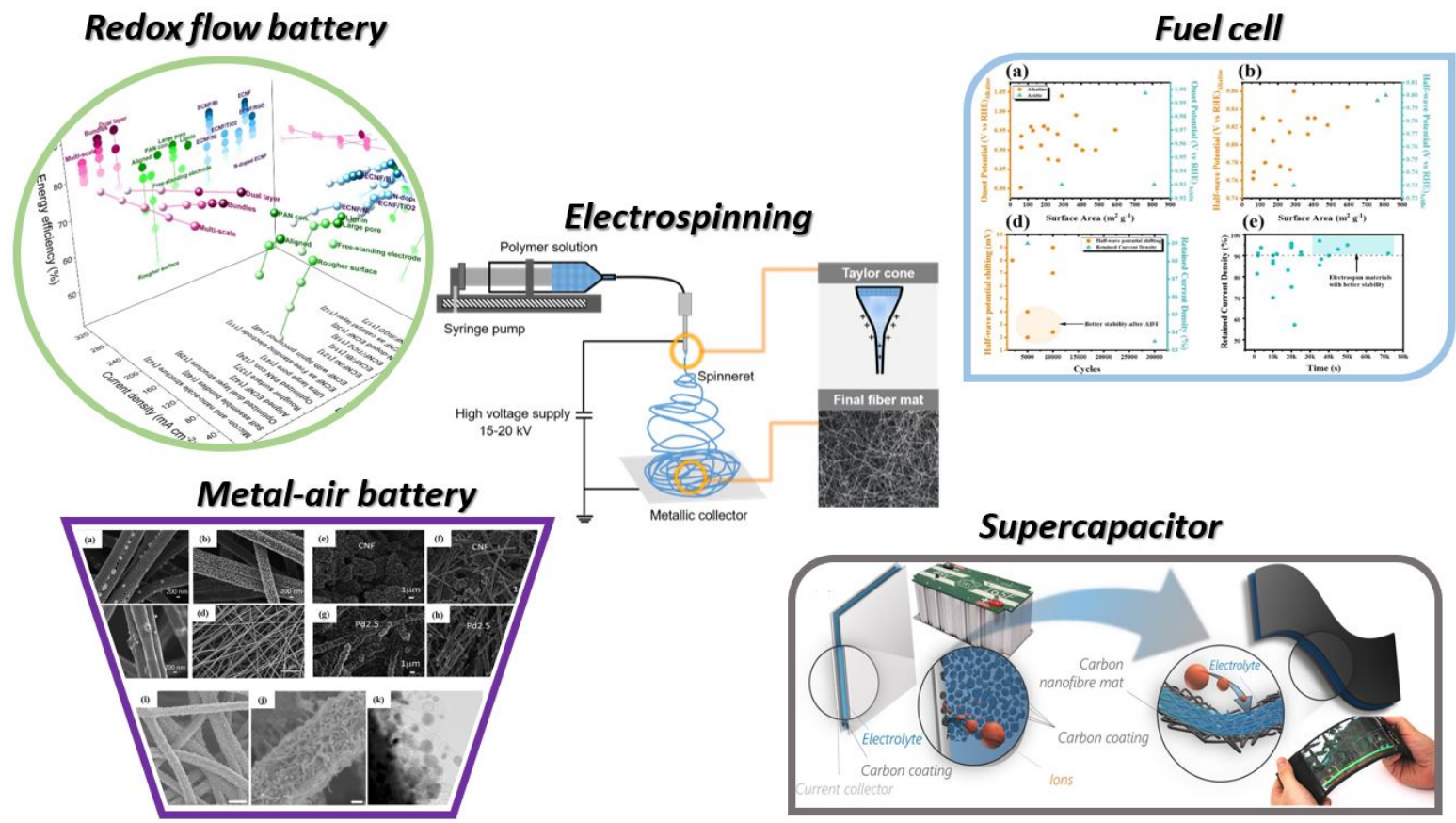

Electrospinning is a versatile fabrication method for synthesising mesostructured nanofibrous mats and has been explored in this review for the following electrochemical energy storage devices: redox flow batteries, fuel cells, metal air batteries and supercapacitors. 
